\title{
Structure and dynamics in network-forming materials.
}

\author{
Mark Wilson \\ E-mail: mark.wilson@chem.ox.ac.uk \\ Department of Chemistry, Physical and Theoretical Chemistry Laboratory, \\ University of Oxford, South Parks Road, Oxford OX1 3QZ, U.K.
}

\begin{abstract}
The study of the structure and dynamics of network-forming materials is reviewed. Experimental techniques used to extract key structural information are briefly considered. Strategies for building simulation models, based on both targeting key (experimentally-accessible) materials and on systematically controlling key model parameters, are discussed. As an example of the first class of materials, a key target system, $\mathrm{SiO}_{2}$, is used to highlight how the changing structure with applied pressure can be effectively modelled (in three dimensions) and used to link to both experimental results and simple structural models. As an example of the second class the topology of networks of tetrahedra in the $\mathrm{MX}_{2}$ stoichiometry are controlled using a single model parameter linked to the M-X-M bond angles. The evolution of ordering on multiple length-scales is observed as are the links between the static structure and key dynamical properties. The isomorphous relationship between the structures of amorphous Si and $\mathrm{SiO}_{2}$ is discussed as are the similarities and differences in the phase diagrams, the latter linked to potential polyamorphic and "anomalous" (e.g. density maxima) behaviour. Links to both two-dimensional structures for C, Si and Ge and near-two-dimensional bilayers of $\mathrm{SiO}_{2}$ are discussed. Emerging low-dimensional structures in low temperature molten carbonates are also uncovered.
\end{abstract}

\section{Introduction.}

The idea of networks are useful across a huge range of potential applications including electronics, flow charts and even maps [1, 2]. In materials "network" is usually taken to indicate well-defined spatial relationships between individual or collections of atoms which define geometric units. In the crystalline state, for example, well-defined coordination polyhedra (CP) are connected by clear, often relatively simple, symmetry operations which define the overall topology [3, 4]. In $\mathrm{SiO}_{2}$, for example, there exists a range of crystal structures, stable under ambient conditions, which may be understood in terms of repeating $\mathrm{SiO}_{4}$ tetrahedra. However, the way in which these CP link in the different polymorphs differs. This view is not limited to static structure. For example, a range of dynamic properties can be considered in terms of the collective motions of these $\mathrm{CP}$ as fixed units (rigid unit models - RUMs [5]). The problem though is that many, if not most, chemical systems may be described in terms of a network of structure of some 
sort. A more useful definition, perhaps, is to classify network materials as systems in which significant directional connectivity may be identified and is preserved on melting or vitrification into disordered states. Contemplating structure in terms of an underlying network is often traced to Zachariasen [6] although Rosenhein [7] introduced the concept around five years earlier (see reference [8] for a review). The popularity of Zachariasen's work can be traced to his sketch of a two-dimensional network formed from two- and three-coordinate sites. In his picture the network connectivity arises from the percolation in space of relatively simple well-defined structural units ( $\mathrm{MX}_{3}$ triangles). Implicit in Zachariasen's image is that the $\mathrm{MX}_{3}$ ordering in the triangular $\mathrm{CP}$ is imposed by (strong) electrostatic interactions. Indeed, understanding the structure of systems dominated by electrostatic (Coulombic) interactions has a long history [9, 10, 11, 12]. At one level the ionic ordering imposed by the electrostatic interactions might be expected to promote relatively simple structure dominated by ions with opposite charges sitting in nearestneighbour "shells" and in which the coordination number is effectively determined by ion volumes (radius ratio or Pauling's rules). Significant many-body (and hence potentially directional) interactions may complicate this picture. For example, a number of systems of $\mathrm{MX}_{2}$ stoichiometry are dominated by tetrahedral CP at the short-range but show very different topologies as the CP link in different ways.

Alternatively, directionality may be imposed by dominant covalent bonding as observed, for example, in monatomic systems such as carbon, silicon or germanium. Networks obtained for different chemical systems may map onto one another - the structure of glassy $\mathrm{SiO}_{2}$, obtained under ambient conditions, may be mapped onto those for amorphous carbon or silicon $[13,14,15]$ in which the Si-Si (or C-C) links are "dressed" with the O atoms $[16,17,13,18]$. The isomorphology allows both to be considered in terms of a continuous random network [19, 20, 21, 22, 23]. Furthermore, the mapping of one network onto another may be non-trivial. In $\mathrm{Si}$ and $\mathrm{SiO}_{2}$, for example, we anticipate very different dominant bonding interactions. However, this isomorphology is not automatic. Systems such as $\mathrm{BeCl}_{2}, \mathrm{GeSe}_{2}, \mathrm{GeO}_{2}$ and $\mathrm{ZnCl}_{2}$ can be considered (at ambient pressures) as related to $\mathrm{SiO}_{2}$, being constructed from $\mathrm{MX}_{4}$ tetrahedral CP. The key differences lie in the connectivity of these tetrahedra, in particular the bridging angles $\left(\theta_{M X M}\right)$ between neighbouring tetrahedra $\left.[15,24,25,26]\right)$. Furthermore, these detailed network topologies are controlled by a range of different factors including temperature and pressure. The thermodynamic metastability of amorphous or glassy configurations means that the detail of preparation conditions may also be a significant. To further complicate matters, neutron, Raman and Mössbauer studies on $\mathrm{GeSe}_{2}$ highlight the formation of homopolar bonds (effectively like atoms on a nearest-neighbour length-scale) [27, 28, 29, 30] which breaks the simplistic picture of networks formed from CP (as in Zachariasen's picture).

Despite the relative simplicity of this description of their structure, the systems listed display an interesting richness of physical properties. $\mathrm{SiO}_{2}$ is a strong liquid (Angell's classification [31]) as the viscosity change on cooling follows an Arrenhius behaviour. Both $\mathrm{ZnCl}_{2}$ and $\mathrm{GeSe}_{2}$ appear more fragile (exhibiting significant non- 
Arrenhius behaviour). Liquid strength may be quantified using a fragility index, $m$, with a low numerical value indicative of strong behaviour. Stronger liquids (fragility index $m=20-28$, e.g. $\mathrm{SiO}_{2}, \mathrm{BeF}_{2}$ and $\mathrm{GeO}_{2}$ [32]) show relatively obtuse $\mathrm{M}-\mathrm{X}-\mathrm{M}$ bond angle distributions $\left(\bar{\theta}_{\mathrm{MXM}}=155-120^{\circ}\right)$ and are dominated by corner-sharing units $[33,34]$. The more fragile liquids $(m=30-60)$ are $\mathrm{ZnCl}_{2}, \mathrm{ZnBr}_{2}$ [35], $\mathrm{GeS}_{2}$ [36] and $\mathrm{GeSe}_{2}$ [37] which show $\bar{\theta}_{\mathrm{MXM}}=120-85^{\circ}[38,39,40,33,28]$. Overall, therefore, the obtuse M-X-M angles equate with topologies built from corner-sharing CP whilst, M$\mathrm{X}-\mathrm{M}$ angles $\leq 120^{\circ}$ stabilise edge-sharing units [25]. Below angles of $\simeq 120^{\circ}$ the fragility increases as the number of edge-sharing units increases [41].

A recurring theme in networks constructed in this manner is the presence of ordering on multiple length-scales (highlighted by the presence of more than one peak in the scattering functions) and attributable to the topology of the linked tetrahedra, for example, intermediate length-scale ordering (IRO, ordering beyond the short-range length-scale which is effectively imposed by the ion radii). IRO is characterised in diffraction patterns as a first sharp diffraction peak (FSDP) which appears at scattering angles equivalent to $k_{\mathrm{FSDP}} \simeq 1-1.5 \AA^{-1}$, compared with typical principal peak positions of $k_{\mathrm{PP}} \simeq 2.1-2.7 \AA^{-1}[42,43]$. FSDPs often appear narrower than the corresponding principal peak and hence might be expected to correspond to the longest range ordering. Recent diffraction measurements have, however, highlighted the presence of extendedrange ordering which shows oscillations of periodicity $2 \pi / k_{\mathrm{PP}}[44,38,40]$.

The ring size distributions, which characterises the distribution of shortest paths, represents a natural "language" for discussing longer-range ordering [45]. Changing conditions by applying temperature or pressure may change the network topology (and ring size distributions) both by modifying the spatial relationships of the $\mathrm{CP}$ and controlling the CP geometries themselves (i.e. changing the tetrahedra). However, although the ring structure provides a useful mathematical framework for quantifying network topology, observing the actual rings themselves remains elusive, although their presence may be inferred from (structural) diffraction data (via a detailed analysis of the pair distribution functions) or from Raman spectroscopy (see ref. [46] and references therein).

Interest in network materials is increasing for a number of reasons. Improvements in experimental diffraction techniques are expanding the range of phase space which can be accessed [47]. Improvements in analysis techniques, in particular in terms of modelling the extracted pair distribution functions, are increasing the obtained spatial resolution $[48,49]$. Evidence from fluctuation electron microscopy [50, 51, 52, 53], following from earlier transmission electron microscopy studies [54], and ellipsometry [55] indicates that the CRN picture for systems such as a-Si is not unique in describing the pair distribution functions, with nanocrystalline ensembles equally able to model these functions [56]. In addition, there has been significant growth in the range of metal-organic framework (MOF) materials obtained experimentally [57, 58, 59]. In a MOF the networks are composed of metal atoms joined by organic molecules and, as a result, the links between the metal centres are controlled by the organic molecules acting as bridges. As a result, 
the organic molecules in the MOFs behave as the bridging atoms in systems such as $\mathrm{SiO}_{2}$. The ability to control the bridging organic molecule (to a potentially far greater extent than for the single atoms bridges) means that there is a potentially limitless flexibility in network design. Furthermore, developments in synthetic and analytical techniques is making the production and analysis of low-dimensional materials more common. Electron microscopy studies of graphene-derived materials show regions of five-, six- and seven-membered rings which may be considered as amorphous [60, 61, 62, 63, 64, 65, 66]. Thin films of vitreous $\mathrm{SiO}_{2}$, interpreted as constructed from bilayers of $\mathrm{SiO}_{4}$ tetrahedra, have been grown on $\mathrm{Mo}(112)$ [67], $\mathrm{Ru}(0001)$ [68, 69, 70] and graphene [71]. Both of these systems are examples in which the ring size distributions may be observed directly which provides a direct link with the original sketches of Zachariasen. However, the number and size of samples generated to date are relatively small and so the underlying factors controlling the distribution of structures remains unclear.

Simulation models offer potential (partial) solutions to a number of experimental issues. For example, information is available directly from the atom positions as partial structure factors or partial pair distribution functions. Simulations may be deployed as predictive tools allowing extremes of phase space to be probed. However, the effectiveness and validity of these approaches is reliant upon the accuracy and transferability of the potential models. High level electronic structure calculations allow only a small number of atoms to be studied, rendering these methods unsuitable for the study of disordered networks. Density functional theory (DFT) offers a computationally tractable description of the electron density [72] with the detail of the approximations (the density functional, the pseudopotentials, the incorporation of electron correlation effects...) potentially affecting the result (see, for example, the case of $\mathrm{GeSe}_{2}$ in references $[73,74,75,76,77,78,79,80,81,82]$. An alternative is to express interatomic interactions as relatively simple functions of the atom positions. The gross simplification allows for the study of relatively long length- and time-scales. Importantly, the relative weightings of different terms in the models may be varied to uncover the roles of the specific interactions, an approach which is difficult to mimic with electronic structure methods. The disadvantage of using simplified potential models is that their transferability (meaning both in terms of change in state point and chemical identity) may be limited. The use of experimental information (a historic way of obtaining potential parameters) implies a minimisation of the number of those parameters. Whilst making models as simple as possible, this approach risks combining different physical interactions into single terms which may reduce transparency and transferability. Using electronic structure calculations to generate potential parameters [83, 84, 85, 86, 87] allows any number of parameters to be specified. The number of parameters may then be systematically reduced by looking for correlations and coupling terms. 


\subsection{Experimental Background.}

Two key experimental techniques for probing network structure are diffraction (using both X-rays and neutrons) and electron microscopy. The former gives important structural information on macroscopic $\left(N \sim 10^{23}\right)$ bulk, three-dimensional, samples which may be highly averaged (both in space and time). The latter may give detailed structural information (atom positions) for systems in two-dimensions (at surfaces or in sheets). However, the available information, whilst very detailed, is typically available for up to $N \sim 10^{5}$ atoms (i.e. $<<10^{23}$ ). As a result, diffraction experiments may yield fully-averaged information (in the thermodynamic limit) whilst electron microscopy experiments may show highly detailed "snapshots" which must be averaged further to yield proper statistical information.

Key (bulk) structural probes are neutron and X-ray diffraction. A scattering experiment produces a total scattering function, $F^{(z)}(k)$ (where $z=n$ or $X$ for neutrons and X-rays respectively). For a binary system $\mathrm{M}_{m} \mathrm{X}_{x}, F^{(z)}(k)$ may be written in terms of partial structure factors. For example,

$$
F_{1}^{(z)}(k)=c_{M} Z_{M}^{2}\left(S_{M M}(k)-1\right)+c_{X} Z_{X}^{2}\left(S_{X X}(k)-1\right)+2\left(c_{M} c_{X}\right)^{1 / 2} Z_{M} Z_{X} S_{M X}(k),(1)
$$

where $S_{\alpha \beta}(k)$ are Ashcroft-Langreth (AL) structure factors, $c_{\alpha}$ is the mole fraction of species $\alpha$ and $Z_{\alpha}$ is the corresponding coherent neutron scattering length (for neutrons) or the ( $k$-dependent) form factor (for X-rays). The AL functions are an example of atom-atom structure factors (and are related trivially, for example, to the Faber-Ziman functions [88]). The structure factors are related to the corresponding partial radial distribution functions, $g_{\alpha \beta}(r)$, via a Fourier transform, and hence contain information regarding the relative spatial organisation of the atoms, coloured by identity, and averaged over time. However, this deconstruction is not unique. Bhatia-Thornton (BT) functions, for example, break down $F^{(z)}(k)$ into terms dependent upon the system topology $(\mathrm{N})$ and concentration $(\mathrm{C})[89,90]$,

$$
F_{1}^{(z)}(k)=<b>^{2}\left[S_{N N}(k)-1\right]+c_{M} c_{X}\left(b_{M}-b_{X}\right)^{2}\left[\frac{S_{C C}(k)}{c_{M} c_{X}}-1\right]+2<b>\left(b_{M}-b_{X}\right) S_{N C}(k),(2)
$$

where $\langle b\rangle=c_{M} b_{M}-c_{X} b_{X}$ and the BT functions are related to the AL structure factors by

$$
\begin{aligned}
& S_{C C}(k) /\left(c_{M} c_{X}\right)=c_{X} S_{M M}(k)+c_{M} S_{X X}(k)-2 \sqrt{c_{M} c_{X}} S_{M X}(k) \\
& S_{N N}(k)=c_{M} S_{M M}(k)+c_{X} S_{X X}(k)+2 \sqrt{c_{M} c_{X}} S_{M X}(k) \\
& S_{N C}(k) /\left(c_{M} c_{X}\right)=S_{M M}(k)-S_{X X}(k)-\left(c_{M} c_{X}\right)^{-1 / 2}\left(c_{M}-c_{X}\right) S_{M X}(k) .
\end{aligned}
$$

The shape of $S_{N N}(k)$ reflects the system topology regardless of the chemical identities of the species occupying each network "site". $S_{C C}(k)$, on the other hand, reflects chemical ordering on these sites. $S_{N C}(k)$ reflects any correlation between the sites and their occupancy.

The most detailed information which can be extracted from scattering experiments is at a partial structure factor level. Isotopic substitution uses samples which differ 
in their respective isotopic composition and, as a result, in their respective scattering powers. As a result, a second experiment, generating a second total scattering function, $F_{2}^{(z)}(k)$, will differ from $F_{1}^{(z)}(k)$ as the weighting factors in equation 1 will differ. Problematically, extracting full partial structure factor information requires $N(N+1)$ independent experiments for an $N$-species assuming suitable isotope compositions can be synthesised. Furthermore, the potential usefulness of this approach is limited by the constraints of the periodic table. Systems under investigation require abundant and stable isotopes which have significant contrast in scattering length. For $\mathrm{MX}_{2}$ networkforming materials a number of systems have been investigated at a partial structure factor level (with the isotopes used indicated in parentheses); $\mathrm{ZnCl}_{2}\left({ }^{35,37} \mathrm{Cl}\right)[91,44,92]$, $\mathrm{GeSe}_{2}\left({ }^{70,73} \mathrm{Ge},{ }^{N, 76} \mathrm{Se}\right)[44,28,93,27], \mathrm{GeO}_{2}\left({ }^{70,73} \mathrm{Ge}\right)[94,95]$ and $\mathrm{SiO}_{2}\left({ }^{N, 29} \mathrm{Si}\right)[96]$. The respective weightings of the partial structure factors in equations 1 and 2 means that the extraction of the AL or BT functions may be numerically easier depending upon the scattering contrast.

Most commonly the components of a given system will scatter X-rays and neutrons to different extents as the X-rays are scattered by the electron density and the neutrons by the nuclei and so the total structure factors obtained from X-ray and neutron scattering experiments may be usefully combined. From combined X-ray/neutron experiments difference functions can be constructed which eliminate a single partial structure factor. However, a third total scattering function is still required to extract the three partial structure factors. For example, anomalous X-ray scattering allows the third function to be obtained as demonstrated for $\mathrm{GeO}_{2}[97,98,99,100]$. However, the contrast between the X-ray scattering functions is small making extracting the final partial functions difficult in numerical terms.

Improvements in experimental set up has greatly increased the range of both $T$ and $p$ which may be accessed. However, at the more extreme conditions lower quality scattering functions may be obtained with associated difficulties in extracting clear real space information. Furthermore, advances in rapid data acquisition techniques potentially allows the direct monitoring of structural changes on vitrification $[101,102$, 103]. However, the diffraction patterns obtained may again be somewhat noisy requiring significant additional analysis for the effective extraction of structural information. Levitation techniques may be applied to study liquids to high temperature by laserheating the sample as well as into the supercooled regime by removing the laser heating source [103, 104, 105, 106].

The information obtained from these diffraction experiments may highlight the presence of order on a number of length-scales. For example, in $\mathrm{MX}_{2}$ networks ordering is observed on both short- (nearest-neighbour) and intermediate-ranged (intermediaterange order - IRO) length-scales. The presence of IRO is generally associated with the emergence of a feature at low scattering angle (low $k$ ), often termed a first-sharp diffraction peak (FSDP) or pre-peak [42, 43]. Associating features in $k$-space with those in real space is non-trivial. Even if well-resolved partial structure factors are available there are potential transform problems. The geometry of the experimental cell 
may limit the scattering angles for fixed wavelength experiments, truncating the data in $k$-space and potentially leading to spurious oscillations in the real space functions. These problems may become more acute when dealing with data obtained at high pressure (owing to the restrictive cell geometry) or by rapid data acquisition (owing to the relatively low radiation flux). In addition, data obtained from levitation experiments are subject to potential issues arising from sample geometry fluctuations, the effect of the gas jet (in terms of moving the sample or in terms of its oxidising/reducing nature), temperature fluctuations etc.

The development of advanced neutron sources has improved the resolution of the pair distribution functions and uncovered fluctuations extending to high $r$ (and termed extended-range order - ERO) in the systems $\mathrm{ZnCl}_{2}, \mathrm{GeO}_{2}$ and $\mathrm{GeSe}_{2}$ [44]. However, the legitimacy of such results remains controversial as the Fourier transformation procedures are liable to generate unphysical oscillations linked to the truncation of the data in $k$-space.

Electron microscopy studies offer potentially different insight into the detailed structure. The most detailed atomistic information is obtained on surface (or thin film) structure. For $\mathrm{SiO}_{2}$, for example, the microscopy images of the bilayers clearly show the locations of the Si atoms (within an experimental error). As a result, the ring structure significant in terms of discussing the system morphology, is available directly rather than by inference from other information. Overall, therefore, electron microscopy offers potentially superb atomistic detail. However, the typical areas resolved correspond to up to $\sim 10000$ atoms (i.e. $<<10^{23}$ ). As a result, it is unclear if, for example, a ring size distribution obtained from such a relatively small field of vision is truly representative of that obtained over a larger scale. This is, in some sense, the opposite to the information

obtained from diffraction studies which may be highly averaged over both time and space.

Experimental probes of the kind discussed above are able to determine the structural properties of a wide range of systems. There are, however, significant potential problems, for example with closely related systems or structures which may yield similar signals. For example, a random network or nanocrystallite representation may give very similar pair distribution functions (see ref. [8] for a discussion). In addition, potentially polyamorphic systems (such as the $\mathrm{Al} / \mathrm{Y} / \mathrm{O}$ mixtures) show very similar diffraction patterns both as a function of composition and state-point [107].

\section{Models and Modelling Strategies.}

Inter-atomic interactions in condensed phases may be expressed via a cluster expansion

$$
U\left(\left\{\mathbf{R}_{i}\right\}\right)=\sum_{i} \sum_{j} U_{2}\left(\mathbf{R}_{i}, \mathbf{R}_{j}\right)+\sum_{i} \sum_{j} \sum_{k} U_{3}\left(\mathbf{R}_{i}, \mathbf{R}_{j}, \mathbf{R}_{k}\right)+\cdots,
$$

where the terms on the right hand side are the true two-, three-, four-... body interactions. $U_{2}$, for example, represents the true interaction potential, that is, the energy which would result from the interaction between a pair of atoms in an isolated 
atom dimer. To properly express the energy of interaction of a trimer a correction term, $U_{3}$, is added. With these definitions these potential models are completely transferable in the sense that the interaction energy of a given configuration would be exact. However, the time required to calculate the energy of an $n$-body term scales approximately as $N^{n}$ and so the computational tractability of using this expansion scales poorly with the number of terms. In addition the functional forms required to fully describe each energy term may become arbitrarily complex. As a result it is common to truncate the series at the two-, three- or four-body level. Terms of higher order than the pair potential may be incorporated into the two-body term creating an effective pair potential (EPP). The effectiveness of this approach is that the EPPs are both computationally tractable (efficient calculation of the energy of a given configuration) and scale well with system size. Furthermore, the number of parameters required is often small. The price to pay is that the transferability between state points and between different systems may be compromised with potential parameters potentially losing physical meaning. For monatomic systems pair potentials will favour close-packed local environments. As a result, representation of truly directional bonding requires explicit higher order terms. For ionic systems (those dominated by electrostatic interactions) directional interactions may arise from the inclusion of polarization effects.

The balance of the terms in equation 4 is important and tt is possible to reproduce key structural properties (such as bond angle distributions) in a number of ways. In $\mathrm{SiO}_{2}$, for example, the TTAM potential [108, 109] reproduces key static structural properties using pair potentials only. The strategy employed in that case is to subsume the three-body (bond bending) effects into pair potential parameters. In the TTAM potential, for example, the oxygen-oxygen dipole-dipole dispersion coefficient is significantly larger than would be expected from the oxide polarizabilities alone (as determined through the Slater-Kirkwood formula [110]). Much more dramatically, for $\mathrm{ZnCl}_{2}$ the $\mathrm{KDR}$ potential [111] is able to reproduce the relatively acute $\mathrm{Zn}-\mathrm{Cl}-\mathrm{Zn}$ bond angles (or the order of $\sim 110^{\circ}$ ) using pair potential only. In that case the ZnZn dipole-dipole dispersion coefficient is around two orders of magnitude greater than that expected from the polarizability of the zinc cation $\left(C_{6}^{Z n Z n} \sim 7200 \mathrm{au}\right.$ compared with $C_{6}^{Z n Z n} \sim 40$ au obtained from the Slater-Kirkwood formula [110] using $\alpha_{Z n^{2+}} \sim 5$ au [112]. For a relatively small perturbation, such as that employed for the TTAM for $\mathrm{SiO}_{2}$, the EPP is highly successful in reproducing key structural and dynamic properties and, crucially, it retains the computational tractability afforded by pure pair potentials. For the $\mathrm{ZnCl}_{2}$ example, however, the magnitude of the required pair potential enhancement makes the underlying potential terms unphysical and, as a result, affects the system dynamics. For example, the ion vibrational dynamics shown by the KDR potential are very stiff (the low frequency modes are not correctly reproduced) when compared with experiment [113].

A potential advantage of a more simplistic model-based approach (certainly when compared to electronic structure methods) is that specific parameters may be systematically altered allowing the effect of different interactions to be rationalised. A 
disadvantage is that problems which involve (potentially complex) changes in electronic structure (for example, significant electron delocalisation) cannot be trivially considered in this way. Furthermore, problems which involve deviations from simple stoichiometry in systems dominated by electrostatic interactions are potentially difficult to model in this way. For example (in the context of the network systems considered here) a continuous range of stoichiometries for the $\mathrm{Ge}_{x} \mathrm{Se}_{y}$ system can be synthesised but following the evolution of structure across the stoichiometric range requires an electronic structure approach $[114,115,116,117,118,119]$ (see refs. [120, 121] for the latest developments).

Using potential models to study network systems employs two basic strategies.

- Potential models are developed for specific systems, allowing direct comparison with experimental observation.

- Generic models are developed which allow key parameters to be independently modified.

A key to the development of many potential models is the choice of the reference state. For example, choosing an ionic description for the interatomic interactions reflects the usefulness of the well-defined reference state as an ensemble of charged particles. Retaining formal (valence-motivated) ion charges is desirable in terms of presenting a well-defined reference state. However, this strategy becomes problematic when these charges are potentially large [122]. However, important ion properties (e.g. dipole polarizability) are only strictly meaningful if the ion charge has a well-defined value. An alternative strategy is to allow the ion charges to be used as parameters and hence allowing non-integer charges. With non-integer charges model parameters (for example, ion polarizabilities and related dispersion terms) may lose transparency, reducing model transferability between systems or state points.

Three examples are chosen here to highlight different aspects of network structure.

- $\mathrm{SiO}_{2}$ is chosen as a well-studied archetypal network-forming glass and as an example of a system for which a well-developed potential can be used to help interpret experimental results (here, the result of high pressure neutron diffraction experiments).

- A generic model is used to study a range of networks formed by systems of stoichiometry $\mathrm{MX}_{2}$.

- Models for C, Si and Ge are used as examples of potentials employing explicit three-body terms to impose bonding directionality.

\subsection{A Specific "Classic" Network: $\mathrm{SiO}_{2}$.}

From a chemists perspective the bonding in silica can be considered as ionic, a view supported by diffraction experiments which highlight the stability of chargeordered structures in which the nearest-neighbour (short-range order - SRO) lengthscale arises unambiguously from the Si-O correlations (anions surround cations). The 
clear implication is that models in which atoms carry charge, $\left\{\mathrm{Si}^{2 \delta+}, \mathrm{O}^{\delta-}\right\}$, are likely to be most effective.

The most efficient models are based on effective pair potentials. The Fumi-Tosi potential (see ref. [123] and references therein) expresses the interaction between a pair of ions labelled $i$ and $j$ as

$$
U\left(r_{i j}\right)=B_{i j} e^{-a_{i j} r_{i j}}+\frac{Q_{i} Q_{j}}{r_{i j}}-\sum_{n=6,8,10 \ldots} \frac{C_{n}^{i j}}{r_{i j}^{n}} f_{n}\left(r_{i j}\right),
$$

where $B_{i j}$ and $a_{i j}$ are parameters controlling the ion radii and the rate of decay of the repulsive wall respectively, $C_{n}^{i j}$ and $f_{n}\left(r_{i j}\right)$ are the dispersion parameters and respective damping functions (the latter mimicking the loss of asymptotic behaviour in the dispersive interactions at short range [124]), and $Q_{i(j)}$ is the charge on ion $i(j)$. The electrostatic interactions are relatively long-ranged and, therefore, must be carefully accounted for, for example, using an Ewald summation [125]. The exponential form of the short-range energy follows that suggested by Born and Mayer [126] which, in the Fumi-Tosi potential, is written explicitly in terms of the respective ion radii, $\sigma_{i(j)}, B_{i j}=A_{i j} \exp \left[a\left(\sigma_{i}+\sigma_{j}\right)\right]$. The geometries of the local coordination environments (coordination polyhedra) are controlled both by the charges and the relative sizes of the atoms (effectively radius-ratio or Pauling's rules [127]). For $\mathrm{SiO}_{2}$ the relative ion radii promote the formation of structures dominated by four coordinate (tetrahedral) $\mathrm{SiO}_{4}$ local coordination polyhedra under ambient conditions.

EPPs may be parameterised to reproduce key static crystal structures often aided by the typically high crystalline symmetries which may preclude the formation of (low order) induced moments. However, once the site symmetry is broken (most typically by introducing temperature) polarization effects must be incorporated to reproduce dynamic properties such as phonon mode frequencies [123].

The study of silica using potential models has a long history with models developed by reference to experimental data, typically lattice parameters, bulk moduli and phonon model frequencies as well as using the results of electronic structure calculations. $[128,129,130]$ Woodcock et al (WAC) [128] developed a formal valence $\left(\mathrm{Si}^{4+}, \mathrm{O}^{2-}\right)$ charges rigid ion model. The rigid ions means that key bending vibrational frequencies are not reproduced. The addition of many-body effects (ion polarisation) allows these modes to soften whilst retaining the fundamental stretching mode frequencies [131]. Sanders et al [132] developed both rigid-ion and shell models for $\mathrm{SiO}_{2}$ and more generic silicates (see ref. [133] for a review).

An alternative method which also acts to soften the problematic bending mode frequencies is to treat the ion charges themselves as model parameters, see for example the TTAM [108, 109] and BKS [129] potentials (the latter being essentially a reparameterisation of the former). These potentials are parameterised using ab initio calculations. In the DCG [134] the ion charges are allowed to fluctuate in response to changes in the local environment. The TS model [130] is a polarizable-ion model which employs non-integer ion charges. The use of of the BKS, TS and DCG potentials to 
study the phase behaviour of $\mathrm{SiO}_{2}$ has been considered in depth in ref. [135].

\subsection{Generic Models.}

When a pair potential (such as the Fumi-Tosi form shown above) is augmented by a description of ion polarization a many-body character is introduced. Such effects have been incorporated using a shell model (a particular mechanical way of incorporating induced dipoles [136]) or a polarizable-ion model (PIM) in which the induced moments are incorporated directly as point entities [24]. To describe induced dipolar effects the PIM requires two sets of parameters; dipole polarizabilities, $\alpha_{i}$, and short-range damping parameters (SRDPs). The former describe the response of the ion electron density to an electric field (here an internal field arising from the presence of formal charges and other dipoles). The SRDP controls the effect of nearest-neighbour overlap interactions on the induced dipole moments $[85,86,87]$. Dipole polarizabilities can be obtained from $a b$ initio electronic structure calculations (focussed on ions in the condensed phase) [84, 137] or, more historically, by reference to experimentally-determined refractive indices via the Clausius-Mossotti relation [138, 139]. SRDPs can be obtained by performing ab initio calculations on ions confined in crystalline environments and applying specific distortions to the nearest-neighbour shell of counter-ions [85, 86, 87]. For the $\mathrm{MX}_{2}$ stoichiometry PIMs have been developed for $\mathrm{ZnCl}_{2}$ [140, 141], GeSe 2 [140], $\mathrm{GeO}_{2}$ [142], $\mathrm{SiO}_{2}$ [143] and $\mathrm{BeF}_{2}[144,145]$.

As well as models focussed on specific systems, generic models have also been developed. Here the anion polarizability is used as a free parameter which controls the network topology via the M-X-M bond angles [25, 146, 147, 148]. Dipoles induced on the anions which connect a cation pair introduce negative charge in such a way as to screen the repulsive coulombic cation-cation interaction [24]. Increasing anion polarizability, therefore, tends to reduce the mean M-X-M bond angle and in extremis may stablise edge-sharing tetrahedra. The implications of the stabilisation of edge-sharing units are potentially significant. In the $\mathrm{MX}_{2}$ stoichiometry a chain constructed exclusively from edge-sharing units will be charge neutral. As a result, a charge placed at sufficiently long range (meaning a length-scale significantly longer than the anion-cation SRO) will not "see" any overall charge. If the number of edge-sharing units become dominant the system effectively becomes pseudo-polymeric, comprised of charge-neutral chains. The most complex topologies are observed when corner- and edge-sharing units are present in significant amounts, for example, in $\mathrm{GeSe}_{2}[44,28,93,27]$. The ability to control the M-X-M bond angles is important. For $\mathrm{SiO}_{2}$, for example, the Si-O-Si bond angles are relatively obtuse $\left(\bar{\theta}_{\mathrm{SiOSi}} \sim 145^{\circ}\right)$, which corresponds to a charge ordered structure as the more highly charged cations are placed further apart than a corresponding pair of lower charged anions. The charge ordering is reflected in the structure factors. For example, the longer Si-Si nearest-neighbour length-scale is associated with the first peak in the

Si-Si partial structure factor at $k \sim 1.6 \AA^{-1}$. $\mathrm{ZnCl}_{2}$ displays a more acute bridging angles $\left(\bar{\theta}_{Z n C l Z n} \sim 110^{\circ}[33]\right)$ corresponding to a reduction in the cation-cation nearest-neighbour 
length-scale which, in turn, leads to the FSDP (and associated IRO) [149].

\section{3. "Covalent" Models.}

For network-forming systems traditionally considered as dominated by covalent interactions (e.g. C, Si or $\mathrm{Ge}$ ) an ionic reference state is clearly inappropriate. In these cases the most simple potential models employed can be broadly understood as a balance between two- and many-body interactions (see ref. [150] for a review). For example, for a model composed of two- and three-body interactions only then the total system energy can be written as

$$
U=\sum_{i, j} U_{2}\left(r_{i j}\right)+\lambda \sum_{i, j, k} U_{3}\left(r_{i j}, r_{i k}, \theta_{i j k}\right),
$$

where $U_{2}$ is the energy of interaction summed over all atoms pairs $i j$ and $U_{3}$ is the threebody term centred on atom $i$. The parameter $\lambda$ controls the difference between the twoand three-body terms. In a monatomic system the two-body term will tend to favour close-packed ( $\sim 12$-coordinate) environments as these maximise the number of nearest neighbours. In the most widely applied models the three-body term acts to destabilise these close-packed environments. In a Stillinger-Weber potential [151], for example, the three-body term acts to energetically bias against the formation of any geometry other than the ideal tetrahedron. The result is that any crystal structures containing bond angles which deviate from that of the ideal tetrahedron become energetically destabilised.

The $\lambda$ term in equation 6 may be used as a free parameter to control the tetrahedricity of the system. The use if $\lambda$ as a free parameter is analogous way to the use of the anion polarizability above in controlling the network topology in the ionic system. The ability to control the relative energetics of the local tetrahedral geometry means that these potentials may be employed as useful model systems to study this generic class of tetrahedral networks $[152,153]$. For example, so-called anomalous properties, such as the temperature of maximum density, may be mapped in terms of $\lambda$ [153]. In addition, raising $\lambda$ generates configurations which resemble amorphous silicon but which can be generated on relatively short simulation time-scales $[154,155]$. The increase in $\lambda$ here effectively drives the system into the amorphous configurations.

\subsection{Generating Amorphous and/or Glassy Configurations.}

A repeating problem in considering network-forming materials is that of generating truly amorphous and/or glassy structures to compare to experiment $\ddagger$. Cooling rates used to extract glassy configurations from the liquid are typically massive by experimental standards (of the order of $10^{15} \mathrm{Ks}^{-1}$ ). The danger, therefore, is that glassy configurations generated solely by liquid cooling reflect the "frozen-in" liquid structure more than any actual structural relaxation accessed prior to reaching the experimental glass transition

$\ddagger$ A glassy configuration being one generated by passing through a glass transition. 
temperature, that is, the glassy structures have not been sufficiently aged. Recent experimental work, using rapid data acquisition, has shown how significant changes in the structure may be observed on vitrification, that is, the glass structure need not represent a simple "frozen liquid" (see, for example, ref. [156] for $\mathrm{SiO}_{2}$ ). Alternative pathways to amorphous configurations may be more accessible to simulation. For example, a number of systems undergo a pressure-induced amorphisation (PIA) [157]. It is possible, given the metastable nature of the amorphous states, that configurations generated in different ways may not have the same averaged structure. The use of either cooling or PIA procedures very much mimic possible experimental procedures. Whilst it is natural to mirror experiment in simulation, models may allow non-experimentally accessible pathways to be followed. For example, the use of an enhanced value of $\lambda$ (see above) for Si effectively drives the system to generate model tetrahedral local environments characteristic of the amorphous state - the enhanced $\lambda$ here effectively accelerates the liquid-amorphous phase transformation. Furthermore, the random pinning of a fraction of the sites mimics the ageing process and allows relatively long time relaxation dynamics to be probed [158].

\section{Network-forming Materials: Three-dimensional Systems.}

To highlight a range of properties we start by considering a number of network-forming systems, starting with those in three dimensions. The starting point is to highlight the structural similarities between a typical monatomic network-former, $\mathrm{Si}$, and a chemically-related system, $\mathrm{SiO}_{2}$. The evolution of the network in $\mathrm{SiO}_{2}$ can then be investigated by the application of pressure, which allows contact to be made with state-of-the-art neutron diffraction studies. Investigating the evolution of structure with pressure is, of course, useful in that the simulation studies effectively mirror a realisable experimental pathway. However, simulations offer the possibility of following alternative pathways which may not be directly experimentally-accessible, but will allow the key underlying interactions to be resolved. Here, for example, we consider the anion polarizability as a free parameter as it can be used to effectively control the interconnectivitiy of the local coordination polyhedra with potentially significant effects on both static structure and dynamics.

\subsection{Isomorphology.}

Significant previous work has focussed on a potential isomorphology between a-Si and $\mathrm{SiO}_{2}$, that is, the concept that typical glassy $\mathrm{SiO}_{2}$ structures may be generated from those for a-Si by placing $\mathrm{O}$ atoms between bonded pairs of $\mathrm{Si}$ atoms (and rescaling the coordinates). Figure 1 highlights some evidence for this isomorphous behaviour. Figures 1 (a) and (b) show the respective structure factors and radial distribution functions for a-Si, calculated using a modified Stillinger-Weber potential [151, 159, 154, 155]. In both cases these functions are compared with the partial analogues $S_{S i S i}(k)$ and $g_{S i S i}(r)$, 
generated from the TS potential [130]. In panels (c) and (d) data in both real and reciprocal space are scaled along the ordinate axis by matching the respective first peak positions in real space. Both $g_{S i S i}(r)$ and $S_{S i S i}(k)$ show a strong mapping of the first two peaks and high $k$ or $r$ oscillations. The most significant differences lie in the intensities of the low $-k$ features which indicates that these systems may differ in their dynamics on intermediate (greater than nearest-neighbour) length-scales.

\subsection{Topology changes with Pressure.}

$\mathrm{SiO}_{2}$ as a key example of a tetrahedral network and which changes topology in a nontrivial way on application of pressure. A key motivation in studying this system is to make contact with state-of-the-art high pressure diffraction experiments [47]. the role of the method of generation on the final glassy configurations is tested by generating configurations by two different procedures. Thermostats are employed to remove kinetic energy (quench) from the liquid state $[160,161]$. As a result the thermostat relaxation time (which couples the system to the heat bath, here kept deliberately cold) controls the rate at which energy is transferred from the system. A thermostat relaxation time of $\tau \simeq 1.2 \mathrm{ps}$, corresponding to a cooling rate of $\sim 10^{15} \mathrm{Ks}^{-1}$. The time between configuration sampling is taken as $\sim 100 \mathrm{ps,} \mathrm{significantly} \mathrm{longer} \mathrm{than} \mathrm{the} \mathrm{liquid}$ relaxation time, meaning that successive configurations are effectively independent. In the second method ambient pressure configurations obtained as described above are cold compressed.

The potential employed is the ionic model derived by Tangney and Scandolo (TS) [130] which employs pair potentials to account for the short-range (overlap) and dispersive interactions as well as reduced ion charges $(\sim-1.38 \mathrm{e}$ and $+2.76 \mathrm{e}$ for the $\mathrm{O}$ and Si ions respectively). Dipole polarisation effects are incorporated (as described in reference [131]) requiring two parameters, the anion polarizability and a shortrange damping parameter (controlling nearest-neighbour overlap effects on the induced dipoles). The model parameters (including the ion charges) are determined by reference to electronic structure calculations.

Figure 2 shows the total neutron structure factors $F^{(n)}(k)$ obtained from the compressed and quenched configurations. These results are compared with those from state-of-the-art neutron scattering experiments [162]. The total structure factors are determined from the partial functions (equation 1) with coherent neutron scattering lengths of $b_{\mathrm{Si}}=4.1491(10) \mathrm{fm}$ and $b_{\mathrm{O}}=5.803(4) \mathrm{fm}$ [163], corresponding to natural isotopic compositions. The partial structure factors themselves are calculated using the atom positions via $S_{\alpha \beta}(k)=<A_{\alpha}^{*}(k) \cdot A_{\beta}(k)>$, where $A_{\alpha}(k)=\frac{1}{\sqrt{N_{\alpha}}} \sum_{i=1}^{N_{\alpha}} e^{i \mathbf{k} \cdot \mathbf{r}_{i}}$ are the fourier components.

The TS model appears to give an excellent representation of the experimental neutron scattering data to high pressure, with the data generated by compression giving closer agreement with experiment. The difference between the simulated and 
experimental data can be quantified by a quality of fit parameter,

$$
<\Delta F^{2}>^{1 / 2}=\frac{1}{N_{Q}}\left\{\sum_{i=1}^{N_{Q}}\left[F^{e x}(Q)-F^{s i m}(Q)\right]^{2}\right\}^{1 / 2},
$$

where $F^{e x}(Q)$ and $F^{s i m}(Q)$ are the total scattering functions obtained from experiment and simulation respectively. $\left\langle\Delta F^{2}\right\rangle^{1 / 2}$ is shown as a function of pressure in figure 2 and highlights firstly the better agreement obtained using the compressed configurations and secondly that the quality of fit appears largely pressure independent. The most significant differences between the compressed and quenched configurations appear at $k \sim 1.6 \AA^{-1}$ (the location of the first peak) which is a reflection of different IRO resulting from the connectivity of the $\mathrm{SiO}_{4}$ tetrahedra.

As discussed earlier, the distribution of ring sizes is a useful way of considering structure on length-scales longer than those imposed by short-range ordering. Figure 3(a) shows the mean ring size changes with increasing pressure. To pressures of $p \sim 18 \mathrm{GPa}$ the mean ring size increases for the quench-generated configurations but falls for the compressed analogues. Previous work indicates models preserving the $\mathrm{SiO}_{4}$ the integrity of the tetrahedra show a rise in mean ring size with pressure [164, 45]. However, if the local coordination geometries are allowed to change then a more complex change in the ring size distribution emerges [165, 166, 167, 168, 169]. At higher pressures both methods show a fall in $\langle n\rangle$. Whilst the ring size distributions (and their means) represent a natural language to discuss IRO they are not directly observed in three dimensions. However, coordination numbers are accessible by integrating the first peak in the total radial distribution function. In systems dominated by ionic interactions this peak can reasonably unambiguously be assigned to the cation-anion coordinations. Figure 3(b) shows the pressure evolution of the cation coordination environments for the compressed configurations. At ambient pressures four-coordinate Si sites dominate. As the pressure is increased there is a rise in five-coordinate sites whilst, at yet higher pressures, the number of six-coordinate Si sites increases. Figure 3(c) shows how the mean ring size relates to the mean coordination number. The key observation is that the reduction in the mean ring size is clearly correlated with the increased mean coordination number (and hence the increase in the number of five- and six-coordinate Si sites).

A key, therefore, is to link an experimental observable (the mean coordination number) to an important indicator of IRO (the change in the ring size distribution).

3.2.1. Ring Closure Model. The mean ring size of the starting (ambient pressure) configurations is $\langle n\rangle$, where

$$
<n>=\frac{\sum n p_{n}}{\sum p_{n}}
$$

where $p_{n}$ is the number of rings of size $n$. The total number of rings is $\sum p_{n}=N_{\text {tot }}$. One ring closure converts one ring into two rings of mean size $(<n>/ 2)+1$. 
As a result, the new mean ring size $\left\langle n^{\prime}>\right.$ becomes

$$
\left(N_{\text {tot }}+1\right)<n^{\prime}>=\left(N_{t o t}-1\right)<n>+2\left\{\frac{<n>}{2}+1\right\} .
$$

For $m$ such closure events,

$$
\begin{aligned}
\left(N_{t o t}+m\right)<n^{\prime}> & =\left(N_{t o t}-m\right)<n>+2 m\left\{\frac{<n>}{2}+1\right\}, \\
& =N_{t o t}<n>+2 m,
\end{aligned}
$$

and so,

$$
<n^{\prime}>=\frac{N_{t o t}<n>+2 m}{N_{t o t}+m} .
$$

The number of closure events, $m$, can be related to the number of $n$-coordinate sites as

$$
m=N_{\text {cat }} \sum_{n=5}^{\infty}(n-4) f_{n},
$$

where $N_{\text {cat }}$ is the number of cations in the system and $f_{n}$ is the fraction of $n$-coordinate sides. $(n-4)$ is the number of ring closures required to form an $n$-coordinate site starting from a four-coordinate site. Substituting equation 12 into equation 11 gives

$$
\begin{aligned}
<n^{\prime}> & =\frac{N_{t o t}<n>+2 N_{c a t} \sum_{n=5}^{\infty}(n-4) f_{n}}{N_{t o t}+N_{c a t} \sum_{n=5}^{\infty}(n-4) f_{n}}, \\
& =\frac{4<n>+2 \sum_{n=5}^{\infty}(n-4) f_{n}}{4+\sum_{n=5}^{\infty}(n-4) f_{n}},
\end{aligned}
$$

where we have taken $N_{\text {tot }}=4 N_{\text {cat }}$.

The mean $\mathrm{Si}-\mathrm{O}$ coordination number is given by

$$
\bar{n}_{\mathrm{Si}}^{\mathrm{O}}=\sum_{n=1}^{\infty} n f_{n} .
$$

Noting that

$$
\sum_{n=1}^{\infty} f_{n}=1
$$

and rewriting $\bar{n}_{\mathrm{Si}}^{\mathrm{O}}$ as

$$
\bar{n}_{\mathrm{Si}}^{\mathrm{O}}=4 \sum_{n=1}^{\infty} f_{n}+\sum_{n=5}^{\infty}(n-4) f_{n},
$$

leads to

$$
\bar{n}_{\mathrm{Si}}^{\mathrm{O}}=4+\sum_{n=5}^{\infty}(n-4) f_{n} .
$$

Substituting into equation 14 gives

$$
<n^{\prime}>=\frac{4<n>+2\left(\bar{n}_{\mathrm{Si}}^{\mathrm{O}}-4\right)}{\bar{n}_{\mathrm{Si}}^{\mathrm{O}}},
$$


which finally links the mean ring size and mean coordination number. Figure 3 highlights the the strong correlation between the ring closure model results and those derived using the configurations obtained under compression.

Figure 4 shows the pressure-evolution of the partial structure factor, $S_{S i S i}(k)$. The low $-k$ feature $\left(k_{1} \sim 1.60 \AA^{-1}\right.$ at ambient pressure $)$ corresponds to ordering on an intermediate length-scale $\left(r_{1} \sim \frac{2 \pi}{k_{1}} \sim 4 \AA\right)$ whist the second peak $\left(k_{2} \sim 2.70 \AA^{-1}\right.$ at ambient pressure) corresponds to a nearest-neighbour length-scale $\left(r_{2} \sim 2 \AA\right)$. The inset to the figure shows these two length-scales as a function of pressure. The nearestneighbour length-scale contracts slightly on pressurisation whilst the most significant changes are seen in the intermediate length-scale.

\subsection{Topology changes with model parameters.}

The work described in the previous section highlights how the evolving topology with pressure can be understood for a single system, $\mathrm{SiO}_{2}$. There are, however, a number of key $\mathrm{MX}_{2}$ stoichiometry systems which are related to $\mathrm{SiO}_{2}$ in the sense that they can be usefully considered as formed by linked tetrahedral coordination polyhedra under ambient conditions. These systems differ critically in the mean bond angles for the $\mathrm{M}-\mathrm{X}-\mathrm{M}$ triplets (i.e. involving the bridging anion) and hence may differ in topology on length-scales beyond the nearest-neighbour SRO. For example, $\mathrm{BeF}_{2}\left(\sim 155^{\circ}[33]\right)$, $\mathrm{GeO}_{2}\left(\sim 130^{\circ}[34]\right), \mathrm{ZnCl}_{2}\left(\sim 115^{\circ}[33]\right), \mathrm{GeSe}_{2}\left(\sim 85^{\circ}[38,40]\right), \mathrm{BeCl}_{2}\left(\sim 80^{\circ}[33]\right)$, and $\mathrm{SiSe}_{2}\left(\sim 80^{\circ}[33]\right)$.

The previous discussion regarding $\mathrm{SiO}_{2}$ demonstrates how the evolution of structure on application of a particular perturbation, in that case pressure, can be monitored and rationalised. An alternative strategy is to systematically vary a potential model parameter and observe how structure evolves. In a PIM, for example, the anion polarizability $\alpha$ dominates the M-X-M bond angles (see section 2.2) and so varying a single model parameter allows the model to be systematically changed from generating $\mathrm{SiO}_{2}$-like structure to $\mathrm{BeCl}_{2}$ - or $\mathrm{SiSe}_{2}$-like structure. Again, this mirrors the discussion from earlier. The pressurisation of $\mathrm{SiO}_{2}$ represents an experimentally accessible pathway, whilst the systematic variation of the anion polarizability is a method of modifying the topology not directly accessible (although the results may intersect with experimental observation).

Figures 5a-c shows the evolution along the $T=1000 \mathrm{~K}$ isotherm of the AshcroftLangreth partial structure factors, $S_{X X}(k), S_{M M}(k)$ and $S_{M X}(k)$, as a function of anion polarizability. $S_{X X}(k)$ is dominated by a principal peak which shifts only slightly from $k \sim 1.95 \AA^{-1}$ at $\alpha=0$ (corresponding to a rigid-ion model) to $k \sim 1.98 \AA^{-1}$ at $\alpha=25 \mathrm{au}$. $S_{M X}(k)$ shows a shift in the principal peak position from $k \sim 1.90 \AA^{-1}$ at the rigidion limit to $k \sim 2.00 \AA^{-1}$ at $\alpha=25$ au and significant change at low scattering angles $\left(k<k_{P P}\right)$ with a low intensity peak at $k \sim 1.40 \AA^{-1}$ at low $\alpha$ shifting to lower $k$ $\left(k \sim 1.05 \AA^{-1}\right)$ at higher $\alpha$. Perhaps the most significant changes occur to $S_{M M}(k)$. At low $\alpha S_{M M}(k)$ shows a single principal peak at $k \sim 1.55 \AA^{-1}$, compared with the 
analogous peak in $S_{X X}(k)$ at $k \sim 1.95 \AA^{-1}$, reflecting the charge-ordered nature of the system. As $\alpha$ is increased the principal peak effectively "splits" into a FSDP (at $k \sim 1 \AA^{-1}$ ) and a principal peak (at $k \sim 2 \AA^{-1}$ ). Above $\alpha \sim 22.5 \AA^{-1}$ the the FSDP position effectively "saturates" at $k \sim 1 \AA^{-1}$.

Further insight into the network topologies can be gained by further sub-dividing the cation (metal) density according to their local bridging environment [77, 78, 79, 80, 81, 82]. In this scheme cations are labelled in terms of their connectivity. Units which show only corner-sharing are labelled "0", a single edge-share "1" and two edgeshares " 2 ". Figure 5e shows the evolution of the fractions of these sites as $\alpha$ increases. Up to $\alpha \simeq 10$ au the network topology is dominated by corner-sharing units (labelled "0"). As $\alpha$ increases beyond $\alpha \sim 10$ au edge-sharing units begin to be stabilised in units with simultaneous corner-sharing ("1"). At the highest polarizabilites fully edge-sharing units become stable.

Figure 6a-c shows the analogous Bhatia-Thornton structure factors as a function of $\alpha$. The "three peak" structure in $S_{N N}(k)$ typical of network-forming melts $[90,170]$ and shows a FSDP independent of anion polarizability. The FSDP in $S_{N C}(k)$ occurs in concurrence with that in $S_{N N}(k)$ (as noted by Salmon [90]). The FSDP in $S_{C C}(k)$ increases in intensity with $\alpha$. The emergence of the FSDP in $S_{C C}(k)$ can be understood by considering the balance of terms in equation 3. $S_{X X}\left(k_{F S D P}\right) \sim 0$ and so $S_{C C}\left(k_{F S D P}\right)$ reflects the interplay between $S_{M M}\left(k_{F S D P}\right)$ and $S_{M X}\left(k_{F S D P}\right)$, which both show an FSDP which shifts to low $k$ on increasing $\alpha$. However, the intensity of $S_{M M}\left(k_{F S D P}\right)$ increases more rapidly than $S_{M X}\left(k_{F S D P}\right)$. There is no formal requirement for FSDP suppression in $S_{C C}(k)$. In a system of charges only $S_{C C}(k)$ is proportional to the charge-charge structure factor, $S_{Z Z}(k)$, as $S_{Z Z}(k)=\left(c_{A} c_{B}\right)^{-1} S_{C C}(k)$. Whilst the system must maintain electroneutrality (effectively the charges must sum to zero) there is no imposition over what length-scale must encompass this charge balance. Despite this, and observations from neutron diffraction studies which show the presence of a FSDP in $\mathrm{GeSe}_{2}$ and, to a lesser extent, in $\mathrm{ZnCl}_{2}$ [44], the presence of a FSDP in $S_{C C}(k)$ remains a subject of debate $[82,78,171,172]$. One key reason for the continued debate is that different models, or level of treatment, produce subtly different results. For example, potential models for $\mathrm{GeSe}_{2}$ constructed as a formal sum of two- and three-body terms show no clear FSDP $[173,174,175,176]$. Furthermore, the results of density-functionalbased electronic structure calculations differ depending on subtle calculation detail. Non-self-consistent methods show a clear FSDP $[73,74,75,76]$ but using either a local or gradient-corrected density approximation shows no such feature [77, 78, 79, 80, 81, 82]. Polarizable-ion simulations show the intensity of $S_{C C}\left(k_{F S D P}\right)$ to be related to the fraction of edge-sharing units $[74,141,172]$. The key, therefore, is that the fine balance between stabilised corner- and edge-sharing units may be shifted significantly by subtle differences in model or in the level of theoretical treatment, and so may generate sufficiently different topologies reflected in the difference in FSDP intensity. In support of this hypothesis, closer inspection of the pair distribution functions indicates that the "GeSe ${ }_{2}$ " structures $[173,174,175,176]$ are more akin to those associated with $\mathrm{GeO}_{2}$ in 
being more charge-ordered and hence corresponding to a system with low $\alpha$ on figure $6(\mathrm{c})$, which has only a hint of low $k$ shoulder on the principal peak of $S_{C C}(k)$.

The network topology can be further characterised by defining six partial structure factors "coloured" by the cation label,

$$
S_{M M}(k)=\sum_{a=0}^{2} \sum_{b=0}^{2} x_{a} x_{b} S_{M M}^{a b}(k),
$$

where $\{a, b\}=\{0,1,2\}$ are the cation labels and $x_{a(b)}$ is the fraction of sites with label $a(b)$. Figure 7a-f shows the six partial structure factors as a function of $\alpha$. The FSDP appear most intense in functions with edge-sharing character $(\{a, b\}=\{1,2\}) . S_{M M}^{02}(k)$ shows little oscillation at $k>k_{P P}$, as the $\{0,1,2\}$ definitions preclude a ' 0 ' site being directly linked to a ' 2 ' site. The rise in intensity as $k \longrightarrow 0$ at low $\alpha$ in $S_{M M}^{11}(k)$ and $S_{M M}^{12}(k)$ corresponds to a "phase separation", that is, a breakdown of the percolating edge-sharing network. The rise in intensity at $k \longrightarrow 0$ in $S_{M M}^{00}(k)$ indicates a "phase separation" corresponding to a breakdown in an exclusively corner-sharing network. The rise in intensity as $k \longrightarrow 0$ is can be linked with a percolation threshold in the fraction of a given species approaching a half [177].

In order to connect directly with the results of diffraction experiments, figure 8 shows the radial distribution functions, $g_{\alpha \beta}(r)$, obtained from neutron diffraction experiments on $\mathrm{ZnCl}_{2}$ [44], $\mathrm{GeSe}_{2}$ [44] and $\mathrm{GeO}_{2}$ [94, 95], compared with those obtained from simulation using different anion polarizabilities. The functions are scaled in terms of the first peak position in $g_{M X}(r)$. to allow effective direct comparison with experiment. As a result the first peak position in $g_{X X}(r)$ shifts to higher $r$ as the nearest-neighbour anion-cation length-scale contracts slightly as the anion polarizability increases. The ratio of the first peak positions, $r_{X X}(r) / r_{M X}(r)$ indicates these systems as dominated by tetrahedral networks. $g_{M M}(r)$ shows the greatest changes with $\alpha$ as highlighted in figures $8(\mathrm{c})$ and (d). For $g_{M M}(r)$ the ratio of the first peak positions is $r_{M M} / r_{M X} \sim 2$ at low $\alpha$, compared with $r_{X X} / r_{M X} \sim 1.6$, indicative of a charge-ordered structure. As $\alpha$ increases the first peak in $g_{M M}(r)$ is shifted to lower $r$ corresponding to a reduction in the M-X-M bond angle. At high $\alpha$ the first peak positions in $g_{M M}(r)$ and $g_{X X}(r)$ coincide. At the highest $\alpha r_{M M} / r_{M X} \sim 1.4$ as a significant number of edge-sharing units form.

The changes in the simulated $g_{M M}(r)$ functions very much reflect those observed experimentally. Here, $\mathrm{GeO}_{2}$ represents a system with relatively obtuse M-X-M (GeO-Ge) bond angles (of the order of $\sim 130^{\circ}$ ) with $\mathrm{ZnCl}_{2}$ and $\mathrm{GeSe}_{2}$ having more acute angles (of the order of $\sim 110^{\circ}$ and $\sim 85^{\circ}$ respectively). The significant differences in the experimental functions for $g_{S e S e}(r)$ in the glassy and liquid states is interesting and highlights the potentially significant changes which may occur on vitrification (the glass structure does not necessarily reflect that of a "frozen liquid"). In this case it is likely that the glassy state contains more edge-sharing units frozen in on cooling. 
3.3.1. What is a first-sharp diffraction peak? A clear message from the consideration of the network structure of the $\mathrm{MX}_{2}$ systems (both from experimental and simulation results) is that it is the cation density which shows the most dramatic differences between systems, in particular in terms of the emergence of intermediate-range order, most clearly characterised via the FSDP position. Given this we need to be clear as to the potential origin of the FSDP, which we may define as a peak which appears in the total scattering function at scattering angles lower than those associated with the principal peak. At low polarizability the FSDP at $k \sim 1.5 \AA^{-1}$ arises as the result of charge ordering. The greater cation formal charge (compared with the anion charge) is reflected in a longer nearest-neighbour cation-cation length-scale when compared with the anion-anion analogue. As a result, the first peak in $S_{M M}(k)$ appears at lower $k$ (corresponding the longer wavelength oscillations in real space) then the corresponding peak in $S_{X X}(k)$. As $\alpha$ increases the position of the FSDP moves to $k \sim 1 \AA^{-1}$, which corresponds to ordering an intermediate length-scales. In real space the increase in $\alpha$ shifts the first peak in $g_{M M}(r)$ to around that of the first peak in $g_{X X}(r)$ and, at even higher $\alpha$, to even shorter length-scales. As $\alpha$ increases the principal peak in $S_{M M}(k)$ shifts to higher $k$ corresponding to a density wave in the cation density resulting from the reduction of the nearest-neighbour length-scale. This reduction in length-scale is commensurate with depletion of cation density on an intermediate length-scale and so corresponds to the formation of the FSDP $[178,179]$. At even higher $\alpha$ the principal peak in $S_{M M}(k)$ shifts to higher $k$ corresponding to a cation-cation nearest-neighbour length-scale now shorter than that for the anions and the FSDP position effectively "saturates" at $k \sim 1 \AA^{-1}$. The saturation results from the changes in network topology arising from the formation of edge-sharing units. Peaks at $k<1 \AA^{-1}$ can be imposed by templating the network by introducing relatively large (organic) molecules which favour cavity formation and generates length-scales longer than those imposed by the ions alone [180]. The key property of these networks lies in their topological flexibility. As a result, systems with even relatively large cavities are only marginally worse off (in terms of energetics) than more dense structures. As a result, the low thermodynamic driving force, coupled with potentially high kinetic barriers for topological reconstruction, renders these structures relatively stable (as exemplified in the wealth of zeolite chemistry).

Figure 9 shows schematic chains of cations in order to demonstrate how ordering emerges. If the atoms are evenly spaced (corresponding to a charge-ordered system in which repulsive coulombic interactions dominate this length-scale) then a single lengthscale dominates. Reducing the nearest-neighbour cation-cation length-scale in pairs (mimicking the effect of anion polarizability) simultaneously introduces both short and relatively long (intermediate-ranged) length-scales. These changes are reflected in the effective "splitting" of $S_{M M}(k)$ at low $\alpha$ into a principal and first-sharp diffraction peak at higher $\alpha$. The figure also highlights how templating may introduce even longer lengthscales. The position and width of any low $-k$ features generated by the addition of the template will depend on the size and shape of the templating molecule.

The presence of peaks at relatively low scattering angles is, of course, not limited 
to these network systems. The addition of $\mathrm{RbCl}$ to $\mathrm{ZnCl}_{2}$, for example, leads to a significant enhancement of the FSDP as the concentration of $\mathrm{RbCl}$ increases [149]. The $\mathrm{RbCl}$ acts as a network-modifier which promotes formation $\mathrm{ZnCl}_{4}^{2-}$ tetrahedra which act as large anions. As a result, the emerging "FSDP" is a normal principal peak which arises from the mixing of the $\mathrm{Rb}^{+}$and $\mathrm{ZnCl}_{4}^{2-}$ ions.

Overall, we identify three key "mechanisms" by which FSDPs form in ionic systems:

- Charge-ordered. In the $\mathrm{MX}_{2}$ stoichiometry systems such as $\mathrm{SiO}_{2}$ and $\mathrm{BeF}_{2}$ and, in the $\mathrm{MX}_{3}$ stoichiometry, many metal trihalides [181, 182, 183, 184, 185] shows FSDPs at $k \sim 1.3-1.7 \AA^{-1}$. The dominant mechanism is the relatively high cation charge which promotes a longer cation-cation nearest-neighbour length-scale then the corresponding anion-anion scale. As a result, the "FSDP" is actually a principal peak in $S_{M M}(k)$ which appears at a relatively low scattering angle.

- Many-body. $\mathrm{ZnCl}_{2}, \mathrm{MgCl}_{2}$ and $\mathrm{GeSe}_{2}$, for example, show a FSDP at $k \sim 1 \AA^{-1}$ $[91,44]$ which arises from induced anion dipoles (many-body effects) perturbing the cation sub-density away from a more charge-ordered structure as described above. The formation of edge-sharing units causes the FSDP position to become saturated at $k \sim 1 \AA^{-1}$.

- Size. The seeming universality of principal peaks at $k \sim 2 \AA^{-1}$ simply reflects typical inter-atomic length-scales (i.e. of the order of $1-2 \AA$ ). Molecular ions, which can, to a first approximation, be considered as large spherical ions, impose their own lengthscales which will be a function of their effective diameters (see, for example, the low temperature molten salts in refs. [186, 187, 188, 189, 190]). Recent work, focussed on seemingly "simple" carbonates and sulphates and in which sample levitation is employed in order to study both the melt and vitreous states, is highlighting how this picture of the molecular ions as large spheres may break down, in particular at low temperatures as the glass transition temperature is approached [191, 192].

3.3.2. Extended-Range Order. As discussed in section 1.1 neutron diffraction studies have indicated the presence on extended-range ordering (ERO) in a range of $\mathrm{MX}_{2}$ systems [44]. These observations, however, remain controversial as careful data processing is required to prevent spurious oscillations entering the generated real space distribution functions. Simulation results are also potentially vulnerable to system size effects which can generate spurious oscillations in the pair distribution functions. Importantly, these results are not susceptible to oscillations arising from the Fourier transformation of data truncated in $k$-space since they are generated directly from of the pair separations.

Figure 10 shows the Bhatia-Thornton pair distribution functions generated at three polarizabilities and displayed as $r h_{I J}^{\mathrm{BT}}(r)\left(h_{I J}^{B T}(r)=g_{I J}^{B T}(r)-1,\{I, J\}=\{N, C\}\right)$ and deconstructed into contributions from the three weighted $r h_{\alpha \beta}(r)$ distribution functions.

$$
h_{N N}(r)=\frac{1}{9} h_{M M}(r)+\frac{4}{9} h_{X X}(r)+\frac{4}{9} h_{M X}(r)
$$




$$
\begin{aligned}
& h_{C C}(r)=h_{M M}(r)+h_{X X}(r)-2 h_{M X}(r) \\
& h_{N C}(r)=\frac{1}{3} h_{M M}(r)-\frac{2}{3} h_{X X}(r)+\frac{1}{3} h_{M X}(r) .
\end{aligned}
$$

Figure 10 highlights the oscillations which extend to high $r$ in all cases and which are consistent with experimental observation [44]. The oscillation length-scales (frequencies) in the atom-atom functions are consistent with those associated with the respective partial structure factor principal peak positions. At intermediate $\alpha$ the three $S_{\alpha \beta}(k)$ functions show near-coincident principal peak positions and so each $r h_{\alpha \beta}(r)$ shares a common oscillation length-scale, $a_{1}=2 \pi / k_{\mathrm{PP}}$ with the oscillations in $r h_{X X}(r)$ and $r h_{M M}(r)$ in anti-phase to $r h_{M X}(r)$. For $r h_{\mathrm{NN}}^{\mathrm{BT}}(r)$, this cancellation results in a smaller long-range amplitude compared with $r h_{\mathrm{CC}}^{\mathrm{BT}}(r)$ or $r h_{\mathrm{NC}}^{\mathrm{BT}}(r)$. In $r h_{\mathrm{CC}}^{\mathrm{BT}}(r)$, for example, the three functions reinforce resulting in strong oscillations to high $r$. For $r h_{\mathrm{NC}}^{\mathrm{BT}}(r) r h_{M X}(r)$ and $r h_{M M}(r)$ effectively cancel, giving $r h_{\mathrm{NC}}^{\mathrm{BT}}(r) \simeq r h_{\mathrm{XX}}(r)$. At high and low $\alpha$ the principal peak positions in the atom-atom functions are no longer at the same values and so the resulting ERO appears more complex. For example, considering $r h_{\mathrm{CC}}^{\mathrm{BT}}(r)$ (figure 10(b)) in which the three contributions constructively interfere, the effect of the de-phasing of these three contributions is clear.

The ERO can be quantified by fitting the oscillations to $r h_{I J}^{\mathrm{BT}}(r)=$ $2 A_{I J} \exp \left(-a_{0} r\right) \cos \left(a_{1} r-\theta_{I J}\right)$, as suggested by theory [39, 193]. Here $a_{0}^{-1}$ is a decay length, $a_{1}^{-1}$ is the wavelength of the real space oscillations and $\theta_{I J}$ is a phase shift.

3.3.3. The Relationship Between Dynamics and Static Structure. To this point we have considered the static network topologies and observed how these may evolve under a change in either the physical state (pressure) or the model (anion polarizability). It is, however, as yet unclear how these changes may affect the system dynamics. To attempt to make these connections we will consider a number of dynamic observables.

Diffusion.

Diffusion coefficients can be readily determined from the mean-squared displacements and are most easily related to the macroscopic viscosity (although care is required as the motion of the anions and cations is liable to be highly correlated). The change in the viscosity as a function of temperature characterises the liquid fragility (see, for example, ref. [31]). A "strong" glass (e.g. $\mathrm{SiO}_{2}$ ) follows an Arrhenius behaviour (i.e. a plot of $\ln T v \cdot 1 / T$ is linear), corresponding to a gradual structural arrest on cooling, whilst more "fragile" liquids show a significant curvature in the same plot, indicative of a more sudden arrest on cooling.

Figure 11 shows the diffusion coefficients, $D$, obtained for the cations determined from the mean-squared displacements over a range of temperatures and anion polarizabilities for the $\mathrm{MX}_{2}$ stoichiometry. The simulations are performed at two densities, separated into panels (a) and (b). The data are plotted as $\ln D v \cdot 1 / T$ to highlight curvature, characteristic of the liquid fragility and fitted to a Vogel-FulcherTammann (VFT) law, $D=D_{0} \exp \left[-B /\left(T-T_{0}\right)\right]$. The VFT fits allow effective glass transition temperatures, $T_{f}$, to be determined, corresponding to $\ln \left(D / D_{0}\right)=$ 
-10. These values for $T_{f}$ scale the temperature axis [194]. In the VFT equation the parameters $B$ and $T_{0}$ control curvature and hence $\left(T_{0} / B\right)$ characterizes fragility $[194,195]$. The insets to both panels in the figure show how $T_{0} / B$ varies with $\alpha$. At both densities, fragility increases at $\alpha>15$ au. At the higher density an Arrhenius temperature dependence $\left(T_{0} / B=0\right)$ is observed at $\alpha=15$ au. It appears, therefore, that the fraction of edge-sharing species (figure 5(e)) effectively correlates with the system fragility. Systems dominated by edge-sharing units tend towards behaving as relatively fragile (pseudo-polymeric) chains, which are analogous to relatively simple organic molecular systems, expected to be relatively fragile).

Topology.

Figure 11 indicates that the liquid fragility is strongly correlated with the fraction of edge- and corner-sharing units. To monitor the dynamic evolution of the network topology we construct time correlation functions to extract the life-times of specified cation environments (here the corner- or edge-sharing units) as

$$
f(t)=\frac{1}{N_{\text {cat }}} \sum_{i=1}^{N_{\text {cat }}} n_{i}(t) \cdot n_{i}(0),
$$

where $n_{i}(t)$ is the " 0 ", " 1 " or " 2 " label for cation $i$. The product $n_{i}(t) \cdot n_{i}(0)$ is defined as

$$
\begin{aligned}
n_{i}(t) \cdot n_{i}(0)=1 & \text { if } \quad n_{i}(t)=n_{i}(0) \\
=0 & \text { if } \quad n_{i}(t) \neq n_{i}(0) .
\end{aligned}
$$

Figure 13 shows $f(t)$ for $n_{i}=2,1$ and 0 respectively for $\alpha=22.5 \mathrm{au}$ at five temperatures (from $T=800$ to $1500 \mathrm{~K}$ ). As the temperature is lowered the sites labelled " 2 " transform relatively slowly indicating that the residence times associated with these edge-sharing units are becoming significantly longer than those associated with corner-sharing units.

\section{Relaxation.}

As the liquid is cooled towards the glass transition the relaxation time diverge until, at $T=T_{g}$, the liquid is unable to reach thermodynamic equilibrium in a "reasonable" time-scale. The relaxation dynamics may be probed by, for example, inelastic neutron scattering which generates dynamic structure factors, $S(k, \omega)$, which may be transformed from both $k$ - and $\omega$-space to generate real space (van Hove) functions, $G(r, t)$ (see below). Figure 12, for example, shows the intermediate scattering functions, $F_{M M}(k, t)$ ("intermediate" in the sense of being between $S(k, \omega)$ and $G(r, t)$ ), which show characteristic short-time $(\beta)$ and longer-time $(\alpha)$ relaxation. $F_{M M}\left(k_{X}, t\right)$ is calculated at two polarizabilities, $\alpha=15 \mathrm{au}$ and $25 \mathrm{au}$ just above the respective melting points, along the $T=800 \mathrm{~K}$ isotherm. These two polarizabilities are highlighted as examples of systems in which the corner- and edge-sharing units dominate the structure respectively (figure $5(\mathrm{e})$ ). The functions are shown at $k$-values corresponding to the positions of the principal $(\mathrm{X}=\mathrm{PP})$ and first-sharp diffraction ( $\mathrm{X}=\mathrm{FSDP}$ ) peaks. The $\beta$-relaxation regime is characteristic of the motion of the ions within their nearestneighbour "cages" and, as a result, appears significantly stronger in the principal 
peaks (which correspond to the SRO). The $\alpha$-relaxation time-scales are fitted to stretched exponential functions of the form $F_{M M}^{f i t}\left(k_{X}, t\right)=A \exp \left[(-t / \tau)^{\gamma}\right]$. At the higher polarizability the time-scale for $\alpha$-relaxation on the length-scale associated with the FSDP is considerably longer than that associated with the principal peak as would be expected ("de Gennes narrowing" [196]). As the system is cooled, therefore, the presence of density fluctuations on the intermediate length-scale controls the vitrification dynamics in contrast to systems which arrest at the glass transition temperature due to pure packing effects in which it is dynamics of the principal peak length-scale which dominate.

Figure 11 highlights how the fraction of edge-sharing units is correlated with the liquid fragility (a measure of how the liquid dynamics changes on cooling) whilst figure 13 shows how the edge-sharing units are more long-lived at lower temperature (just above $T_{g}$ ). To further probe this we can construct van Hove spatial correlation functions "coloured" by cation environment. Van Hove correlation function contains information regarding the inter-atomic arrangements and may be divided into "self" and "distinct" terms, the former containing information as to how individual atom locations evolve, and the latter information as to how the correlations between different atoms evolve. At short times the distinct terms will resemble the respective static pair distribution functions but will become less structured as time increases reflecting the averaging out associated with diffusive motion. Here, distinct van Hove correlation functions are defined by

$$
G_{\alpha \beta}\left(\mathbf{r}^{\prime}, \mathbf{r}, t\right)=\left\langle\rho_{M}^{\alpha}(\mathbf{r}, 0) \cdot \rho_{M}^{\beta}\left(\mathbf{r}^{\prime}, t\right)\right\rangle,
$$

where $\rho_{M}^{\alpha}(\mathbf{r}, 0)=\frac{1}{N_{\alpha}} \sum_{i=1}^{N_{\alpha}} \delta\left[\mathbf{r}-\mathbf{r}_{i}(t)\right]$ and $\alpha$ and $\beta$ refer to the "2", "1" or "0" labels which corresponds to six functions. Each function contains information as to the spatial evolution of a particular coloured site with respect to another.

The six van Hove correlation functions are shown in figure 12, generated along the $T=1000 \mathrm{~K}$ isotherm at $\alpha=22.5 \mathrm{au}$ (in the regime with significant fractions of edge-sharing units). The positions of the first peaks in the respective functions vary, a reflection of the longer metal-metal length-scales associated with the different local environments. $G_{02}(r, t)$ shows no nearest-neighbour length-scale peak, reflecting the geometric constraint that a ' 0 ' site cannot connect directly to a ' 2 '. The highlighted length-scale at $r \sim 6 \AA$ is associated with the FSDP and appears both relatively stable and associated with the presence of edge-sharing units.

Overall, therefore, the underlying static structure appears heavily correlated with key dynamic properties.

\subsection{Polyamorphism.}

A number of network-forming systems display potentially polyamorphic behaviour [197]. The presence of a density-driven liquid-liquid phase transition is often associated with the melting curves gradients (the Clapeyron slope - $d T_{m} / d p$ ). A significant number 
of systems show negative slopes and maxima in their melting curves, for example, Cs, Ba, Eu, $\left.\mathrm{H}_{2} \mathrm{O}[198,199,200]\right)$. At ambient pressure both $\mathrm{Si}$ and Ge show a negative $d T_{m} / d p$ implying a maximum at negative pressures which may be accessible [198, 199, 201, 202, 203]. Simulation studies show the carbon phase diagram to be similarly re-entrant, albeit at significantly higher pressures [204, 205, 206, 207].

Figure 14(a) shows the liquid/diamond crystal coexistence obtained for carbon, generated using a Tersoff-II potential model [208] for a pressure range of $p \sim 100-$ 2200GPa. Figure 14(c) shows the analogous phase diagram for Si determined for the Stillinger-Weber potential. For carbon a liquid $\leftrightarrow$ amorphous transition curve can be identified by performing a series of MD simulations in which the system is subjected to a cooling/heating cycle along a series of isobars [204]. By monitoring the local geometry (in particular through the tetrahedral parameter, q) and the diffusion coefficients, $D$, a locus of temperatures at which both $\langle q\rangle$ and $D$ change discontinuously can be identified. The amorphous state corresponds to so-called "tetrahedral amorphous" carbon $[209,210,211,212,213,214,215,216,217,218]$. This locus of points identifies the liquid/amorphous "phase" boundary (as highlighted in figure 14(a)). Furthermore, the transition shows a hysteresis which effectively defines the spinodal limits, the temperature range of which increases with pressure.

The entropy changes form melting or amorphisation can be obtained from the slopes of the coexistence curves, $\Delta S_{X}(p)=\frac{d p}{d T} \Delta V_{X}(p)$, where $X$ labels the transition and $\Delta V_{X}(p)$ is the associated volume change. $\Delta V_{X}(p)$ and $d p / d T$ can be determined directly from simulation. The entropy changes at around the coexistence curve turning points are $\Delta S_{\text {melt }} \sim 30.1 \mathrm{JK}^{-1} \mathrm{~mol}^{-1}$ and $\Delta S_{l-a} \sim 18.2 \mathrm{JK}^{-1} \mathrm{~mol}^{-1}$ respectively. These correspond to values of $\Delta S_{\text {melt }}$ for $\mathrm{Si}$ and $\mathrm{Ge}$ of 27.1 and $24.8 \mathrm{JK}^{-1} \mathrm{~mol}^{-1}$ respectively [219]. A value of $29.3 \mathrm{JK}^{-1} \mathrm{~mol}^{-1}$ is estimated for $\mathrm{C}$ from the Si and Ge data [220].

Two state mixing models predict the existence of a liquid-liquid critical point based on the mixing of high- and low-density local coordination environments (high-density liquid and low-density liquid - HDL and LDL respectively) [199, 200, 221], $T_{c}=W / 2 R$, where $R$ is the gas constant and $W$ is a mixing parameter. For Si $T_{c} \sim 1060 \mathrm{~K}$ at $p \sim-1 \mathrm{GPa}[222,223,224,199]$. At $T<T_{c}$ the LDL and HDL states are separated by a coexistence curve with an associated first order phase transition, which is shown in figure 14(c) as predicted for Si from the two-state model. The entropy change estimated for the Si LDA $\longrightarrow$ HDA transformation is $22 \mathrm{JK}^{-1} \mathrm{~mol}^{-1}$ [225].

Figure 14(e) shows the respective simulated melting curves for Si and C scaled into reduced units. Under scaling the curves become near-coincident, confirming these systems as corresponding states. The figure also shows the effect of performing the same scaling of the carbon liquid/amorphous and silicon LDA/HDA coexistence curves. In both cases the respective spinodals are shown and scaled in the same way [225, 224]. Whilst the respecting liquid/crystal coexistence curves become near-coincident, the coexistence curves separating the different disorder states show significant differences. For example, the linear nature of the Si LDA/HDA coexistence curve reflects its origin in the two-state model [199]. The carbon liquid/amorphous coexistence curve shows 
a significant curvature, attributable to the massively larger pressure range associated with the coexistence (the pressure range is of the order of $1500 \mathrm{GPa}$ for $\mathrm{C}$ compared with $\sim 20 \mathrm{GPa}$ for $\mathrm{Si}$ ).

Figure 14(d) shows the LDA/HDA coexistence curve determined from a PIM for $\mathrm{ZnCl}_{2}$ [226] compared with that suggested by experiment [227]. In this case the coexistence curve is determined by performing a series of pressurisation (and depressurisation) simulations and monitoring key structural properties, here the cation coordination environments. For the $\mathrm{ZnCl}_{2}$ model the low- to high-density transformation is the result of a fundamental change from a system dominated by four-coordinate cations to one in which six-coordinate sites are in the majority (as suggested previously for $\left.\mathrm{SiO}_{2}[228]\right)$.

The phase diagram shown suggests differences when compared to the analogous diagrams for $\mathrm{Si}, \mathrm{H}_{2} \mathrm{O}$ and $\mathrm{SiO}_{2}$ (table 2). For $\mathrm{ZnCl}_{2}$ the glass transition temperature is lower than the critical temperature, analogous to the observations for $\mathrm{H}_{2} \mathrm{O}$ and $\mathrm{Si}$ but not for $\mathrm{SiO}_{2}$ (see ref. [228] and references therein). Furthermore, $T_{c}$ is higher than the melting point $\left(T_{m} \sim 591 \mathrm{~K}\right)$, different to $\mathrm{H}_{2} \mathrm{O}$, Si and $\mathrm{SiO}_{2}$, although this behaviour is predicted for carbon [229]. For a two-state model $[200,230] T_{c}=825 \mathrm{~K}$ corresponds to a non-ideal mixing parameter, $W=\sim 13.7 \mathrm{kJmol}^{-1}$. Generally speaking, one expects $T_{c}<T_{m}$ as a reflection of the magnitudes of (say) the LDA/HDA and melting transitions. $T_{c} / T_{m}$ values show that this is the case for $\mathrm{H}_{2} \mathrm{O}$, $\mathrm{Si}$ and $\mathrm{SiO}_{2}$, but not for $\mathrm{ZnCl}_{2}$. $\mathrm{ZnCl}_{2}$ is, however, recognised as anomalous with a relatively low melting enthalpy $\left(16.1 \mathrm{kJmol}^{-1}[231], 9.8 \mathrm{kJmol}^{-1}\right.$ [232]) which would be similar to the enthalpies associated with the LDA/HDA phase change. The predicted entropy change $\left(\Delta S \sim 1.6 \mathrm{JK}^{-1} \mathrm{~mol}^{-1}\right)$ is significantly smaller than that associated with melting $\left(\Delta S \sim 16.6 \mathrm{JK}^{-1} \mathrm{~mol}^{-1}[232]\right)$.

\section{5. "Anomalies".}

A range of network systems display an array of anomalous structural and dynamic properties. Examples include the $\mathrm{MX}_{2}$ systems $\left(\mathrm{SiO}_{2}, \mathrm{GeO}_{2}\right.$ and $\left.\mathrm{BeF}_{2}\right)$ and the monatomic systems ( $\mathrm{Si}$ and $\mathrm{Ge}$ ) as well, of course, as $\mathrm{H}_{2} \mathrm{O}$. Perhaps the most celebrated anomaly the density maximum observed with increasing temperature and most wellknown for $\mathrm{H}_{2} \mathrm{O}$ (see, for example, ref. [233] and references therein). The locus of the temperatures at which the turning points are observed in the $p T$ plane defines the temperature of maximum density (TMD) line. Although the existence of a TMD is the most widely-known anomaly, there are numerous others which may often be inter-related by simple thermodynamic arguments. For example, anomalies appear in both the heat capacity and isothermal compressibility. Significantly, perhaps, anomalies are also observed in the diffusivities, a dynamic property more difficult to relate directly to the thermodynamic anomalies $[234,235,236,237,238,239,240,241,242$, 243, 244, 245, 246, 247, 172, 248, 249, 250]. The anomalous properties have been mapped for accurate models of $\mathrm{SiO}_{2}[239,240,172,250], \mathrm{BeF}_{2}$ [248, 249, 250], $\mathrm{GeO}_{2}$ 
[250] and $\mathrm{H}_{2} \mathrm{O}[235,236,237,238,250]$ as well as traced in the Stillinger-Weber potential (see section 2.3) in terms of the parameter $\lambda$ (which, recall, controls the magnitude of the three-body interaction) $[152,153]$. However, determining the specific relationships between anomalies in different systems remains elusive, although clear similarities have been observed. For example, both the tetrahedrality of the local CP (meaning the coordination number and/or the geometry) and their interconnectivity have been found to be significant. In related work ramp potentials, which impose two energetically favourable length-scales, have also been employed [251, 252, 253, 172]. The relative importance of changes in the local CP against changes in the topology (the interconnectivity of the CP) remains unclear. The work of Jabes et al [250] clearly shows how the different anomalies relate for the specific systems listed above. However, systematic differences are difficult to "unpick" as the different specific models are not trivially related in terms of their parameterisation.

\section{Network-forming Materials: Two dimensional glasses.}

Zachariasen [6] pioneered the idea of considering the structure of a generic glass as a network of corner-sharing $\mathrm{MX}_{3}$ triangles (stoichiometry $\mathrm{M}_{2} \mathrm{X}_{3}$ ). Zachariasen's motivation was, of course, generating a memorable and distinctive image constrained to a two dimensional page. However, structures of the sort shown in his original image are envisaged as representing typical three-dimensional structure in bulk systems such as $\mathrm{B}_{2} \mathrm{O}_{3}$ [3]. This system is unusual in displaying a layered crystal structure based on three-coordinate $\mathrm{B}$ atoms, as well as a polymorph based on linked $\mathrm{BO}_{4}$ tetrahedra [3]. Furthermore, experimental evidence indicates that structures such as boroxyl rings (based on $\mathrm{B}_{3} \mathrm{O}_{3}$ hexagonal units) survive into the liquid and glassy phases (see, for example, [254, 255, 256, 257, 258, 259]). However, more direct examples of Zachariasen's original two-dimension motif are observed in reduced graphene oxide $[260,63]$. Understanding two-dimensional structure, which implicitly leads to structural control, allows connections to be drawn with network theories [261] and may potentially answer key questions which are general to three-dimensional systems as well. For example, it may be possible to construct hyperuniform materials which display no density fluctuations beyond a certain length-scale [262, 263].

Perhaps the most widely studied two-dimensional system is graphene which, as a crystal, can be considered as built from purely hexagonal units and which may have potentially interesting physical and electronic properties [264, 265]. Two- or quasi-twodimensional structures are attractive as their dimensionality favours their imaging by electron microscopy. In a typical experiment the electron beam energy is controlled in such a way as to maximise resolution whilst not significantly perturbing the structure under investigation. The electron beam may be utilised in a more active sense by increasing its energy to deliberately disrupt the material structure [61,62]. For graphene, therefore, this procedure would allow access to amorphous graphene (a-G) structures. Further to this, microscopy images show the presence of amorphous graphene (a-G) 
characterised by five-, six- and seven-membered rings [60, 61, 62, 63]. Full experimental control of the distribution of the respective ring sizes has yet to be achieved with different strategies employed, for example, using the electron beam to "melt" the ideal graphene $[65,66]$ or using chemical vapour deposition to deposit an amorphous layer [64]. The modelling strategy developed here mirrors that for three dimensional carbon [204] by generating configurations across a range of stresses from a knowledge of the underlying two-dimension phase diagram [266, 267].

To appreciate the conditions under which amorphous structures may form it is useful first to consider potential two dimensional crystal structures. Figure 15(a) shows the energies of four key crystal structures as a function of area. These are the hexagonal lattice (constructed from three coordinate atoms and equivalent to a graphene sheet), a close-packed lattice (constructed from six coordinate atoms), a square lattice (constructed from four coordinate atoms) and a lattice built from percolating octahedra and squares (also constructed from three coordinate atoms). The hexagonal net is energetically favoured with both the close-packed and 4/8 lattices potentially stable at relatively low and high areas (high and low stress) respectively.

Whilst graphene is a well-known structure of carbon, the silicon and germanium analogues (silicene and germanene respectively) are less well understood. Silicene has been grown on Ag substrates [268, 269, 270, 271] whilst both Si and Ge sheets have been the subject of theoretical investigation [272, 273, 274]. The difficulty in obtaining either silicene or germanene experimentally can be attributed to the relative energetics of the puckered or planar forms. In both cases the puckered form is energetically very close to the the planar which will tend to promote continued growth to form the (thermodynamically stable) diamond crystal structure. Figure 15(b) shows the energy/area curves for $\mathrm{C}$, Si and Ge determined in both the planar and puckered configurations. The energy for $\mathrm{C}$ is calculated with a Tersoff-II potential whilst those for Si and Ge both use Stillinger-Weber potentials. At each area the energy is determined for a hexagonal sheet and for a fully relaxed sheet (i.e. allowed to pucker). For both Si and Ge the puckered forms are stable over the planar, consistent with the results of electronic structure calculations $[272,273,274]$. The area is determined by projection onto a plane and so the energy minima for the puckered structures lie at lower areas than those for the planar nets. As the system becomes stretched (high area) the planar forms become stable. For Si (Ge) the energy of the puckered form is $\sim 15.0(7.5) \mathrm{kJmol}^{-1}$ lower than the planer form with energy barriers (for planar $\longrightarrow$ puckered) of 36.3 (32.7) $\mathrm{kJmol}^{-1}$. In the case of carbon these values are significantly larger with the planar form (the graphene sheet) being favoured by $\sim 419.1 \mathrm{kJmol}^{-1}$ with a barrier of $\sim 509.2 \mathrm{kJmol}{ }^{-1}$. As a result, the formation of planar silicene or germanene will require stabilisation, for example, by deposition on a surface or by being placed under tension. Furthermore, the relatively large energy barriers for both $\mathrm{Si}$ and $\mathrm{Ge}(>>R T)$ indicate potential metastability for these forms. 


\subsection{Amorphous Structures.}

Figure 14(b) shows the graphene sheet/liquid two dimensional phase diagram determined from direct simulations of the liquid/crystal interface (mimicking the threedimensional results in section 3.4) [204]. The coexistence curve becomes re-entrant at high stress mimicking the three dimensional diamond/liquid coexistence curve (figure 14(a)) [204] and corresponding to transitions from the crystal to a more dense liquid. Re-entrant behaviour of the form shown in the figure is also displayed by a gaussian core model (GCM) [275, 276, 277, 278] in both two- and three-dimensions [279, 280, 275, 281]. Figure 14(b) shows how the GCM crystal/fluid coexistence curve may be mapped onto the carbon coexistence curves by varying the two GCM parameters (which control the width and energy scale of the repulsive gaussian function). The mapping of the GCM coexistence curve onto that for carbon appears more impressive in two- compared to three-dimensions. The hexagonal lattice crystal structure displays a dual relationship with a close-packed lattice [282]. An alternative model for controlling the amorphous net of five-, six- and seven-membered rings is, therefore, to consider effective interactions between the centres of mass of each ring which will form a near close-packed array favoured by the GCM. In three dimensions the mapping of the coexistence curves is less impressive as any analogous dual relationship is less well-defined. Indeed, it is the body-centred crystal which is thermodynamically stable over the wide density range covering the re-entrant liquid/crystal coexistence curve.

Amorphous structures can be generated by rapidly cooling from the liquid state at fixed volume. the determination of the liquid/crystal coexistence curve means that, at any given stress, quenches can be initiated at a set temperature above the melting point. Nosé-Hoover thermostats $[160,161]$ are employed to control the rate of cooling. Figure 15(a) shows the energies of the final amorphous structures as a function of area. For $A \lesssim 4 \AA^{2}$ the graphene crystal remains energetically stable over the amorphous configurations. However, the energies of the amorphous configurations are favoured over the $4 / 8$ crystal which implies a potential formation pathway between the two (see below). Figure 16 shows molecular graphics "snapshots" at different stresses. Panels (a) and (b) correspond to areas $A \sim 1.58 \AA^{2}$ and $A \sim 1.95 \AA^{2}$. At the lowest area (largest stress) the structure is comprised exclusively of close-packed units with the relatively rapid quench rate generating nanocrystalline domains and grain boundaries. At $A \sim 1.95 \AA^{2}$ significant domains of both crystalline hexagonal (graphene-like) and close-packed environments are observed. Again, the dual relationship between the hexagonal net and close-packed environments is significant. The close-pasked lattice can be formed by placing an atom at the centre of a hexagon) means that the grain boundary energies are relatively small, of the order of $10^{-8} \mathrm{Jm}^{-1}\left(\sim 10^{-18} \mathrm{Jatom}^{-1}\right)$. The result is that the formation of a mix of low (three-) coordinate and high (six-) coordinate sites is favoured over forming a more uniform coordination "intermediate" state. Panel (c) shows the effect of performing a much slower cooling at $A \sim 2.15 \AA^{2}$ and in which regions of nanocrystalline graphene are present. Slow cooling at low stress (shown in 
panel (d) and corresponding to $A \sim 2.57 \AA^{2}$ ) shows disordered amorphous structure.

Figure 17 (a) shows the evolution (as a function of area) of the mean coordination number and ring size for both the amorphous and liquid structures. Both the liquid and amorphous systems show a change in mean coordination number from $\sim 3$ to $\sim 6$ ar the area decreases (stress increases) which corresponds to a fall in the mean ring sizem with the changes in the liquid state more gradual with area change. A more detailed breakdown of the changes with area is obtained by observing the fractions of threeto seven-membered rings (figure $17(\mathrm{~b})$ ) which dominate the structures over the stress range studied. At high stress (low area) three-membered rings (which are equivalent to close-packed local environments) dominate. As the area increases three-membered rings are still observed in the liquid but, significantly, are quenched out in forming the amorphous state.

Figure 15 shows the effect of allowing the $4 / 8$ crystal structure to be relaxed fully. About the energy minimum at $A \sim 2.8 \AA^{2}$ and at higher areas the ideal structure relaxes (in which all the $\mathrm{C}-\mathrm{C}$ bond lengths are equal) to structures which have two "long" bonds (which form the four-membered rings) which are joined by one "short" bond. At the energy minimum the bond lengths differ by $\sim 7.5 \%$. At high stress (low area) a stressinduced amorphisation is observed as (under stress) the 4/8 crystal is metastable with respect to the amorphous form.

In order to emphasize any potential effect of formation conditions, figure $17(\mathrm{~d})$ shows the ring size distributions for a-G configurations obtained under a range of consitions. Significantly, when cooling from the liquid the rate of cooling affects the final structures. For example, for the most rapid quenches the close-packed sites (threemembered rings) are retained, that is, the amorphous structure retains significant memory of the liquid state. Similar comments apply to the configurations generated by stress-indiced amorphisation of the $4 / 8$ crystal which show a memory of the crystal structure at the hoghest rates of amorphisation in displaying a relatively large fraction of four- and eight-membered rings. Significantly, however, the ring size distributions obtained from wither the liquid or crystal appear very similar under the slowest cooling regimes.

As is clear from the above, a common problem in obtaining amorphous or glassy configurations is the effective suppression of competing crystallisation. Unequivocal determination of the crystallinity is effectively impossible. Furthermore, in two dimensions the problem is exacerbated as the liquid (and amorphous) configurations are more ordered than the three dimensional analogues. To quantify this statement consider the melting entropies shown in table 1 which compare those obtained for simulated carbon (in both two- and three-dimensions) with those obtained for other model systems in the same dimensionalities [283] and those obtained for Si and Ge in three dimensions [219]. Significantly, the values obtained for C agree with both those for $\mathrm{Si}$ and $\mathrm{Ge}$ (which is expected given their expected conformal relationships) and the ration of the entropies obtained across dimensions are consisten with those obtained for other models. Figures 16 (c) and (d) are visiby different in that the configuration 
shown in panel (c) appears to contain significant domains of crystalline six-membered rings (graphene structure). As a result, the crystallinity can be probed by constructing structure factors and their second moments using from the locations of six-membered rings,

$$
\begin{aligned}
& S_{66}(k)=\left\langle\frac{1}{N_{6}} \sum_{i, j=1}^{N_{6}} \exp \left(i \mathbf{k} \cdot \mathbf{R}_{i j}\right)\right\rangle, \\
& M_{66}^{(2)}(k)=\frac{1}{N_{k}} \sum_{p}^{N_{k}} \frac{\left|A\left(\mathbf{k}_{p}, t\right)\right|^{2}-S_{66}(k)^{2}}{S_{66}(k)^{2}},
\end{aligned}
$$

where $\mathbf{R}_{i}$ defines the ring centre of mass and where the sum is over the $N_{k}$ vectors for $\left|\mathbf{k}_{p}\right|=k$ and where

$$
A\left(\mathbf{k}_{i}, t\right)=\frac{1}{N} \sum_{i, j=1}^{N_{6}} \exp \left(i \mathbf{k} \cdot \mathbf{R}_{i j}\right) .
$$

Figure 16(a) and (b) shows $S_{66}(k)$ and $M_{66}^{(2)}(k)$ for configurations obtained under a range of conditions (varying both area and cooling rate). The increase in intensity of $S_{66}(k)$ as $k \longrightarrow 0$ corresponds to significant clustering of the six-membered rings, and appears stronger for the slower cooling rates. The key observation is that the number of nano-crystallites generated in the final quenched configurations increase as the cooling rates are slowed. Furthermore, although $S_{66}(k)$ can be used as an effective indicator of crystallinity (essentially through considering the peak heights [9]) the second moment contains important additional information regarding the structural anisotropy (which may be particularly useful in the two dimensional environments). A final key observation is that at the highest stresses (lowest areas) crystallisation is much more rapid and difficult to supress.

Finally, figure 16 shows the effect of removing the two dimensional constraint for typical a-G configuration dominated by 5-, 6- and 7-membered rings. At around the energy minimum at $A \sim 2.8 \AA^{2}$ the layer shows a significant rumple.

\subsection{The Aboav-Weaire Law.}

The a-G configurations are dominated by five-, six- and seven-membered rings (figure $17(\mathrm{~d})$ ) if the method of generation is suitably slow to allow the less energetically favourable smaller and larger rings to be quenched out. However, the details of how these rings link (which will define the intermediate-range order in these systems) is, as yet, unclear. A more traditional method for considering the topological arrangement of the rings is though the Aboav-Weaire Law [284, 285] (see also [286] for a review). The original formulation of the Aboav-Weaire Law arose from observations on the structure of packing micro-crystallites (of $\mathrm{MgO}$ ) and epithelia [287, 288]. Aboav [284], for example, noted an inverse relationship between a given ring size and its immediate neighbours, expressed empirically as

$$
<m_{n}>=5+\frac{6}{n},
$$


where $n$ is the central ring size and $\left\langle m_{n}>\right.$ the mean size of the immediate neighbouring rings. Several expanded and modifed forms have been presented [289] (also, see refs. [290, 261, 286] for reviews).

Figure 18 shows the mean neighbouring ring size $<m_{n}>$ against $n^{-1}$ for $A=$ $2.57 \AA^{2}$ highlighting the most statistically-significant region (corresponding to $n=5-7$ ) As the cooling rate decreasesthe relationship becomes more linear, reflecting the smaller number of "defect" carbon sites. The annealing procedure freezes in a small number of defective carbon sites, here meaning those with local two- or four-coordinate local environments. These are particuarly problematic as, once frozen in, the system lacks suitable low energy pathways to facilitate their easy removel using standard molecular dynamics. An alternative is to use a hybrid annealing scheme in which well-known mechanisms are employed to specifically remove these defects. In a T1 mechanism, for example, a four-coordinate site is removed by adding an atom to generate two new threecoordinate sites. In a T2 mechanism the two-coordinate site is simply removed [261]. In both cases the initiaql changes result to some highly non-equilibrium bond lengths and angles and so the system must be re-annealed. Although these procedures do not fully eliminate the defect environments they do greatly reduce the fraction of such sites (to $\sim<1 \%$ ) Figure 18 clearly shows how the expected Aboav-Weaire behaviour is recovered both at slow cooling rates and as the "frozen-in" defects are systematically removed.

\section{Network-forming Materials: "Intermediate" Dimensionalities.}

\subsection{Bilayers of Tetrahedra.}

Significant recent experimental effots have demonstrated the growth of near-two dimensional films of vitreous silica $[68,71]$, interpreted as consisting of bilayers of $\mathrm{SiO}_{4}$ tetrahedra $\left(\mathrm{bi}-\mathrm{SiO}_{2}\right)$ and whose structure may be determined by electron microscopy. The thin vitreous $\mathrm{SiO}_{2}$ films were grown on $\mathrm{Mo}(112)$ [67], $\mathrm{Ru}(0001)$ [68, 69, 70] and graphene [71]. A significant advantage emerging from the microscopy studies is the ability to resolve the detailed ring size distributions as well as the spatial relationships between the rings. As discussed above, ring structure represents a natural language for considering ordering beyond the nearest-neighbour length-scale and yet the rings themselves are rarely directly observed. However, as discussed earlier, the detailed information (here the ring size distributions) is available only for a relatively small subset of the system (i.e. $N<<N_{A}$ ).

5.1.1. Construction method A useful starting point (which allows contact with the work decribed above) is to generate the bilayer configurations from those for a-G $[291,292]$. The simplest approach is to effectively replace each C atom with $\mathrm{Si}$ (which

will eventually become the centre of an $\mathrm{SiO}_{2}$ tetrahedron). The network of silicon atoms defines a framework which may be "dressed" with O atoms place along the Si-Si "bonds". The result of these initial steps in to generate a network or percolated corner- 
sharing $\mathrm{SiO}_{2}$ triangles (overall stoichiometry $\mathrm{Si}_{2} \mathrm{O}_{3}$ ) which, interestingly, is equivalent to Zachariasen's original image [6]. The final steps to generate the bilayer are to pucker the layer (making the Si atoms the apices of corner-sharing pyramids) and then attaching a mirro image through a central layer of bridging $\mathrm{O}$ atoms. The final coordinates must then be scaled for the required Si-O bond length. Figure 19 highlights the transformation procedure taking Zachariasen's original configuration as an exemplar [6].

5.1.2. Models. In addition to utilising a "full" electrostatics-based potential model for $\mathrm{SiO}_{2}$ (here the TS potential discussed earlier, and used to model bulk $\mathrm{SiO}_{2}$ ) a simplified harmonic potential is also considered. The harmonic potential will allow a corner sharing network of perfect tetrahedra to be generated with each tetrahedron able to tilt whilst maintaining the imposed topology. The use of a purely harmonic potential allows separation of the nearest-neighbour geometry (the tetrahedra) from their connectivity, allowing any significance of tetrahedral distortions to be explored. The harmonic potential employed uses harmonic springs netweem four Si-O and six O$\mathrm{O}$ pairs defining each tetrahedron. The use of harmonic potentials in this form allows neighbouring tetrahedra to overlap. To account for this overlap a purely repulsive pair potential (a shifted 24-12 potential) acts between silicon atoms. Since the Si atoms are at the centres of the tetrahedra this potential effectively acts as an inter-tetrahedron repulsive term. The choice of the shifted 24-12 potential,

$$
U(r)=4 \epsilon\left\{\left(\frac{\sigma}{r}\right)^{24}-\left(\frac{\sigma}{r}\right)^{12}\right\}+\epsilon,
$$

allows the inter-tetrahedral interactions to be controlled via the effective diameter, $\sigma$.

5.1.3. Results. Figure 20(a) shows the fully relaxed energies of a single bilayer configuration as a function of the number of $\mathrm{SiO}_{2}$ molecules per unit area (the number density, $\left.n_{0}\right)$. In the absense of any inter-tetrahedral interactions there exists a single critical density (here $n_{0} \sim 20 \mathrm{SiO}_{2} \mathrm{~nm}^{-2}$ ) above which the harmonic energy can always be driven to zero (meaning a configuration in which all the tetrahedra are perfect). The key observation here is that the lack of an inter-tetrahedral interaction means that neighbouring CP are able to overlap, possibly in an unphysical manner, and hence are always able to find a zero energy configuration above a critical density. The same would be true of an array of corner-linked triangles in two dimensions. Figure 20(b) shows the relaxed energies for several bilayer configurations which highlights how the critical density is a function of the topology (system size). This observation highlights how the small "viewing window", although generating information on atomistic lengthscales, is not properly averaged in a thermodynamic sense. For these system sizes (between around 240 and 2000 molecules respectively) the critical density varies between $\sim 19.6 \mathrm{SiO}_{2} \mathrm{~nm}^{-2}$ and $\sim 23 \mathrm{SiO}_{2} \mathrm{~nm}^{-2}$ respectively. An ideal crystalline bilayer (constructed from a graphene sheet and containing six-membered rings only when viewed from above) supports a critical density of $n_{0} \sim 17 \mathrm{SiO}_{2} \mathrm{~nm}^{-2}$. 
Including the shifted 24-12 repulsive interaction (which acts between tetrahedra) partially resolves the problem of generating configurations with unphysical tetrahedral overlaps. The repulsive term introduces an energetic penalty to the overlap which is likely to be more significant at higher densities (greater potential overlap). As a result, the repulsive term defines a "density window" (similar to the "flexibility window" observed for zeolites [293]), defined by upper and lower density limits between which the system energy may be driven to zero, and controlled by the length-scale parameter $\sigma$ in equation 28. Using a model which includes the full electrostatic interactions selects a single preferred density from within this window and, significantly, the value of this density is again dependent upon the system topology.

\section{Molecular ions - Carbonates.}

The a-G and bi-SiO ${ }_{2}$ structures highlighted above represent extreme two- (or near two-) dimensional structures. Similarly, the percolation of edge-sharing tetrahedra highlighted in section 2.2 can be considered as pseudo-one-dimensional structures which are interlinked. Systems with molecular anions have traditionally been viewed as "simple" molten salts with the molecular anion behaving essentially as a large charged sphere. Vibrational spectroscopy on glassy carbonates hints at populations of carbonate species in different environments, resembling those observed in crystalline configurations and those forming part of a network with the cations [294, 295], and implying structure more complex than typical molten salts. Evidence is emerging that the typical structure of these systems may be more complex [191]. For example, combined simulation and high energy XRD experiments show a liquid structure which appears far more complicated than a simple ionic liquid in which the $\mathrm{CO}_{3}^{2-}$ anions form a temperature-dependent low-dimensional network. Earlier models considered the anions as acting as bridges between cations [295]. Figure 21(a) shows the evolution with temperature of the partial carbon-carbon pair distribution function, $g_{C C}(r)$. The most dramatic change occurs at $r \sim 3.3 \AA$. At low $T$ there is a clear peak which transforms into a weak shoulder on heating. Figure 21(d) shows a molecular graphics "snapshot" taken from the simulation at low temperature with bonds drawn between the $\mathrm{C}$ atoms separated by $r \leq 4 \AA$ (i.e. corresponding to the first peak in $g_{C C}(r)$, figure 21(a)). The carbonates form a low-dimensional network of (predominantly) chains. Figures 21(b) and (c) show the evolution of the distribution of chain sizes and the mean chain length as a function of temperature, highlighting the rise in chain length as the system is cooled.

The stability of chain-like structures at low temperature affects the ion dynamics (diffusivities). Furthermore, the significant temperature dependence of the structure indicates that these liquids are extremely fragile, with low temperature liquids showing the increased influence of the carbonate chains. In addition, glassy and/or amorphous forms of carbonates would also be expected to be dominated by these carbonate networks. 


\section{Summary and Conclusions. The Broader Context.}

In this review we have highlighted how the structural and dynamic properties of a range of network-forming materials may be accessed both by experiment and simulation models. Experimental techniques are advancing and thus probing more phase space than previously accessible. In addition, advances are allowing dynamic changes in structure over relatively short time-frames to be observed directly. However, the highly spatially-averaged nature of some results (e.g. from diffraction experiments) and the highly specific nature of other results (e.g. from electron microscopy) present their own interpretative issues. Simulation models may aid interpretation of the experimental results both by modelling specific systems of interest and by allowing systematic changes in key model parameters. By modifying the networks (either in a directly experimentally-realisable way or not) the relationships between spatial ordering on multiple length-scales, as well as isomorphological relationships, can be uncovered. Lower dimensional systems (thin films and single layers) show some analogous structural trends to the three-dimensional cases and, significantly, low-dimensional chains have emerged as candidate structures at low temperature in melts with molecular anions.

\section{Acknowledgement}

It is a pleasure to acknowledge all those who have helped and supported the development of the simulation strategies in both Oxford and at UCL; Dominik Daisenberger, Avishek Kumar, Jessica Long, Paul McMillan, David Robinson, Philip Salmon, Franziska Schäffel, Mahdi Sadjadi, David Sherrington, Mike Thorpe, Jamie Warner, Martin Wilding, Anita Zeidler. 
[1] S.N.Dorogovstev. Lectures on Complex Networks. Oxford University Press, 2010.

[2] L.R.Foulds. Graph Theory Applications. Springer, 1991.

[3] A.F.Wells. Structural Inorganic chemistry. Clarendon, 1984.

[4] U. Mueller. Inorganic Structural Chemistry. Wiley, 1993.

[5] K.D.Hammonds, M.T.Dove, A.P.Giddy, V.Heine, and B.Winkler. Am. Min., 81:1057, 1996.

[6] W.H.Zachariasen. J. Am. Chem. Soc., 54:3841, 1932.

[7] W.Rosenhain. J.Soc.Glass Tech. Trans., 11:77, 1927.

[8] A.C.Wright and M.F.Thorpe. Eighty years of random networks. Phys. Status Solidi B, page 1, 2013.

[9] N.H. March and M.P.Tosi. Coulomb Liquids. Academic Press, 1984.

[10] J.-P. Hansen and I.R. McDonald. Theory of Simple Liquids. Academic Press, New York, 1986.

[11] M.Rovere and M.P.Tosi. Rep. Prog. Phys, 49:1001, 1986.

[12] A.R.Ubbelohde. The Molten State of Matter. John Wiley and Sons, 1978.

[13] A.C.Wright, G.A.N.Conneli, and I.W.Allen. J.Non-Cryst. Solids, 42:69, 1980.

[14] A.Uhlherr and S.R.Elliott. J.Phys.:Condens.Mat., 6:L99, 1994.

[15] M.Wilson, A.Kumer, and M.F.Thorpe. Phys. Rev. B, page submitted, 2012.

[16] W.Y.Ching. Phys. Rev. B, 26:6610, 1982.

[17] J.Samela, S.A.Norris, K.Nordlund, and M.J.Aziz. Nucl. Inst. Meth. Phys. Res., 269:1568, 2011.

[18] A.Uhlherr and S.R.Elliott. J.Phys.:Condens.Mat., 6:L99, 1994.

[19] B.R. Djordjevic and M.F. Thorpe. Computer model of tetrahedral amorphous diamond. Physical Review B, 52:5685-5689, August 1995.

[20] R.L.C.Vink, G.T.Barkema, M.A.Stijnman, and R.H.Bisseling. Phys. Rev. B, 64:245214, 2001.

[21] H. He. J. Non-Cryst. Solids, 89:402, 1987.

[22] N.Mousseau, G.T.Barkema, and S.M.Nakhmanson. Phil. Mag. B, 82:171, 2002.

[23] N.Mousseau and L.J.Lewis. Phys. Rev. Lett., 78:1484, 1987.

[24] P.A.Madden and M. Wilson. Chem. Soc. Rev., 25:339, 1996.

[25] M. Wilson and P.S. Salmon. Phys. Rev. Lett., 103:157801, 2009.

[26] M.Wilson. J. Phys: Condensed. Matter, 24:284114, 2012.

[27] I.T.Penfold and P.S.Salmon. Phys. Rev. Lett., 67:97, 1991.

[28] I.Petri, P.S.Salmon, and H.E.Fischer. Phys. Rev. Lett., 84:2413, 2000.

[29] W.J.Bresser, P.Boolchand, P.Suranyi, and J.P.de Neufville. Phys. Rev. Lett., 46:1689, 1981.

[30] P.Boolchand, J.Grothaus, W.J.Bresser, and P.Suranyi. Phys. Rev. Lett., 25:2975, 1982.

[31] C. A. Angell. J. Non-Cryst. Solids, 73:1, 1995.

[32] V.N. Novikov, Y. Ding, and A.P. Sokolov. Phys. Rev. E, 71:061501, 2005.

[33] J.A.E. Desa. J. Non-Cryst. Solids, 51:57, 1982.

[34] J. Neuefeind and K.-D. Liss. Ber. Bunsenges. Phys. Chem., 100:1341, 1996.

[35] E.A.Pavlatou, S.N.Yannopoulos, G.N.Papatheodorou, and G.Fytas. J. Phys. Chem. B, 101:8748, 1997.

[36] J. Malek and J. Shanvelova. J. Non-Cryst. Solids, 243:116, 1999.

[37] J. Ruska and H. Thurn. J. Non-Cryst. Solids, 22:277, 1976.

[38] P.S.Salmon. J. Phys. Condens. Matter, 17:S3537, 2005.

[39] P.S.Salmon. J. Phys.: Condens. Matter, 18:11443, 2006.

[40] P.S.Salmon. J. Phys. Condens. Matter, 19:455208, 2007.

[41] A. Sartbaeva, S.A. Wells, A. Huerta, and M.F. Thorpe. Phys. Rev. B, 75:224204, 2007.

[42] S.R.Elliott. Nature, 354:445, 1991.

[43] P.S.Salmon. Proc. R. Soc. Lond. A, 445:351, 1994.

[44] P.S.Salmon, R.A.Martin, P.E.Mason, and G.J.Cuello. Nature, 435:75, 2005.

[45] C.S.Marians and L.W.Hobbs. J. Non-Cryst. Solids, 124:242, 1990.

[46] A.Pasquarello and R.Car. Phys. Rev. Lett., 80:5145, 1998.

[47] A.Zeidler and P.S.Salmon. in Molecular Dynamics Dimulations of Disordered Materials. Springer, 2015. 
[48] S.J.L.Billinge and M.G.Kanatzidis. ChemComm, page 749, 2004.

[49] J.M.Gibson. J. Phys.: Condens. Mat., 19:455217, 2007.

[50] M.M.J.Treacy and K.B.Borisenko. Science, 335:950, 2012.

[51] J.M.Gibson, M.M.J.Treacy, T.Sun, and N.J.Zaluzec. Phys. Rev. Lett., 105:125504, 2010.

[52] M.M.J.Treacy and J.M.Gibson. Acta. Cryst. A, 52:212, 1996.

[53] M.M.J.Treacy, J.M.Gibson, L.Fan, D.J.Paterson, and I.McNulty. Rep. Prog. Phys., 68:2899, 2005.

[54] J.C.Phillips, J.C.Bean, B.A.Wilson, and A. Ourmazd. Nature, 325:121, 1987.

[55] X-D.Wang, B.Chen, H-F.Wang, X.Zheng, S-J.Liu, J-B.Wang, B.Li, S-M.Yu, and Z-X.Cui. J. Appl. Cryst., 49:528, 2016.

[56] J.M.Gibson. Science, 335:929, 2012.

[57] A.B.Cairns and A.L.Goodwin. Chem. Soc. Rev., 42:4881, 2013.

[58] T.D.Bennett, D.A.Keen, J-C.Tan, E.R.Barney A.L.Goodwin, and A.K.Cheetham. Ange. Chemie, 50:3067, 2011.

[59] T.D.Bennett, J-C.Tan, Y.Yue, E.Baxter, C.Ducati, N.J.Terrill, H.H.-M.Yeung, Z.Zhou, W.Chen, S.Henke, A.K.Cheetham, and G.N.Greaves. Nat. Comm., 6:8079, 2015.

[60] F Banhart, J Kotakoski, and A.V. Krasheninnikov. Structural Defects in Graphene. ACS Nano, 5:26-41, November 2011.

[61] J.Kotakoski, A.V.Krasheninnikov, U.Kaiser, and J.C.Meyer. Phys. Rev. Lett., 106:105505, 2011.

[62] J.Kotakoski, J.C.Meyer, S.Kurasch, D.Santos-Cottin, U.Kaiser, and A.V.Krasheninnikov. Phys. Rev. B, 83:245420, 2011.

[63] J.C.Meyer, C.Kisielowski, R.Erni, M.D.Rossell, M.F.Crommie, and A.Zettl. Nano. Lett., 8:3582, 2008.

[64] J.Zhao, G.Zhu, W.Huang, Z.He, X.Feng, Y.Ma, X.Dong, Q.Fan, L.Wang, Z.Hu, Y.Lü, and W.Huang. Journal of Materials Chemistry, 22:19679, 2012.

[65] A.W.Robertson, C.S.Allen, Y.Wu, K.He, J.Oliviera, J.Neethling, A.I.Kirkland, and J.H.Warner. Nature Comm., 3:1144, 2012.

[66] J.H.Warner, E.R.Margine, M.Mukai, A.W.Robertson, F.Giustino, and A.I.Kirkland. Science., $337: 209,1985$.

[67] J.Weissenrieder, S.Kaya, J-L.Lu, H-J.Gao, S.Shaikhutdinov, H-J.Freund, M.Sierka, T.K.Todorova, and J.Sauer. Phys. Rev. Lett., 95:076103, 2005.

[68] L.Lichtenstein, C. Buechner, B.Yang, S.Shaikhutdinov, M.Heyde, M.Sierka, R.Wlodarczyk, J.Sauer, and H-J Freund. Angew. Chem. Int. Ed., 51:404, 2012.

[69] M.Heyde, S.Shaikhutdinov, and J.-J Freund. Two-dimensional silica: Crystalline and vitreous. Chem. Phys. Lett., 550:1, 2012.

[70] D.Loeffler, J.J.Uhliruch, M.Baron, B.Yang, X.Yu, L.Lichtenstein, L.Heinke, C.Buechner, M.Heyde, S.Shaikhutdinov, H-J.Freund, R.Wlodarczyk, M.Sierka, and J.Sauer. Phys. Rev. Lett., 105:146104, 2010.

[71] P.Y.Huang, S.Kurasch, A.Srivastava, V.Skakalova, J.Kotakoski, A.V.Krasheninnikov, R.Hovden, Q.Mao, J.C.Meyer, J.Smet, D.A.Muller, and U.Kaiser. Nano. Lett., 12:1081, 2012.

[72] R.G.Parr and W.Yang. Density-Functional Theory of Atoms and Molecules. Oxford University Press, 1995.

[73] M.Cobb, D.A.Drabold, and R.L.Cappelletti. Phys.Rev.B, 54:12162, 1996.

[74] M.Cobb and D.A.Drabold. Phys.Rev.B, 56:3054, 1997.

[75] M.Durandurdu and D.A.Drabold. Phys.Rev.B, 65:104208, 2002.

[76] P.Biswas, D.N.Tafen, and D.A.Drabold. Phys.Rev.B, 71:054204, 2005.

[77] C.Massobrio, F.H.M van Roon, A.Pasquarello, and S.W.De Leeuw. J. Phys.: Condens. Matt., 12:L697, 2000.

[78] C.Massobrio, M.Celino, and A.Pasquarello. Phys.Rev.B, 70:174202, 2004.

[79] C.Massobrio, A.Pasquarello, and R.Car. Phys.Rev.Lett., 80:2342, 1998.

[80] C.Massobrio, A.Pasquarello, and R.Car. Phys.Rev.B, 64:144205, 2001. 
[81] C.Massobrio and A.Pasquarello. J.Chem.Phys., 114:7976, 2001.

[82] C.Massobrio and A.Pasquarello. Phys.Rev.B, 68:020201, 2003.

[83] A.Aguado, L.Bernasconi, and P.A.Madden. Chem. Phys. Lett., 356:437, 2002.

[84] P.W.Fowler and P.A.Madden. Phys. Rev. B, 31:5443, 1985.

[85] P. Jemmer, P.W.Fowler, M. Wilson, and P.A.Madden. J. Chem. Phys., 111:2038, 1999.

[86] C. Domene, P.W.Fowler, P.A.Madden, M. Wilson, and R.J.Wheatley. Chem. Phys. Lett., 333:403, 2001.

[87] C. Domene, P.W.Fowler, P.A.Madden, Jijun Xu, R.J.Wheatley, and M.Wilson. J. Phys. Chem. A, 105:4136, 2001.

[88] H.E.Fischer, A.C.Barnes, and P.S.Salmon. Rep. Prog. Phys., 69:233, 2006.

[89] A.B.Bhatia and D.E.Thornton. Phys.Rev.B, 2:3004, 1970.

[90] P.S.Salmon. Proc: Maths and Phy. Sci., 437:591, 1992.

[91] S.Biggin and J.E.Enderby. J. Phys. C, 14:3129, 1981.

[92] A.Zeidler, P.S.Salmon, S.Philip, R.A.Martin, T.Usuki, P.E.Mason, G.J.Cuello, S.Kohara, and H.E.Fischer. Phys. Rev. B, 82:104208, 2010.

[93] P.S.Salmon and I.Petri. J. Phys.: Condens. Matter, 15:S1509, 2003.

[94] P.S.Salmon, A.C.Barnes, R.A.Martin, and G.J.Cuello. Phys. Rev. Lett., 96:235502, 2006.

[95] P.S.Salmon, A.C.Barnes, R.A.Martin, and G.J.Cuello. J. Phys.: Condens. Mat., 19:415110, 2007.

[96] Q.Mei, C.J.Benmore, S.Sen, R.Sharma, and J.L.Yarger. Phys. Rev. B, 78:144204, 2008.

[97] P.B.Bondot. Acta. Cryst., A30:470, 1974.

[98] P.B.Bondot. Phys. Stat. Sol. (a), 22:511, 1974.

[99] Y.Waseda, K.Sugiyama, E.Matsubara, and K.Harada. Mat. Trans., 31:421, 1990.

[100] D.L.Price, M-L.Saboungi, and A.C.Barnes. Phys. Rev. Lett., 81:3207, 1998.

[101] L.Hennet, I.Pozdnyakova, D.L.Price, G.N.Greaves, M.Wilding, S.Fearn, C.M.Martin, D.Thiaudiere, J-F.Berar, N.Boudet, and M-L.Saboungi. J. Chem. Phys., 126:074906, 2007.

[102] A.C.Barnes, L.B.Skinner, P.S.Salmon, A.Bytchkov, I.Pozdnyakova, T.O.Farmer, and H.E.Fischer. Phys. Rev. Lett., 103:225702, 2009.

[103] C.Benmore, J.K.R.Weber, M.C.Wilding, J.Du, and J.B.Parise. Phys. Rev. B, 82:4202, 2010.

[104] A. Ansell, S. Krishnan, J. J. Felten, and D. L. Price. J. Phys.: Condens. Matter, 10:L73, 1998.

[105] C. A. Angell and S. S. Borick. J. Phys.: Condens. Matter, 11:8163, 1999.

[106] N. Jakse, L. Hennet, D. L. Price, S. Krishnan, T. Key, E. Artacho, B. Glorieux, A. Pasturel, and M.-L. Saboungi. Appl. Phys. Lett., 83:4734, 2003.

[107] M.C.Wilding, M.Wilson, C.J.Benmore, J.K.R.Weber, and P.F.McMillan. PCCP, 15:8589, 2013.

[108] S.Tsuneyuki, M.Tsukada, and H.Aoki. Phys. Rev. Lett., 61:869, 1988.

[109] S.Tsuneyuki, Y.Matsui, H.Aoki, and M.Tsukada. Nature, 339:209, 1989.

[110] N.C.Pyper. Adv. Solid State Chem., 2:223, 1991.

[111] P.N.Kumta, P.A.Deymier, and S.H.Risbud. Physica B+C, 153:85, 1986.

[112] N.C.Pyper, C.G.Pike, P.Popelier, and P.P.Edwards. Mol. Phys., 86:995, 1995.

[113] M.C.C.Riberio, M.Wilson, and P.A.Madden. J. Chem. Phys., 110:4083, 1999.

[114] A.Bouzid, K.J.Pizzey, A.Zeidler, G.Ori, M.Boero, C.Massobrio, S.Klotz abd H.E.Fischer, C.L.Bull, and P.S.Salmon. Phys. Rev. B, 93:014202, 2016.

[115] A.Bouzid, S.Le Roux, G.Ori, C.Tugene, M.Boero, and C.Massobrio. MOLECULAR DYNAMICS SIMULATIONS OF DISORDERED MATERIALS: FROM NETWORK GLASSES TO PHASE-CHANGE MEMORY ALLOYS.

[116] M.Micoulaut, A.Kachmar, M.Bauchy, S.Le Roux, C.Massobrio, and M.Boero. Phys. Rev. B, 88:054203, 2013.

[117] M.Kibalchenko, J.R.Yates, C.Massobrio, and A.Pasquarello. Phys. Rev. B, 82:020202, 2010.

[118] G.Chen, F.Inam, and D.A.Drabold. Appl. Phys. Lett.

[119] F.Inam, D.N.Tafen, G.Chen, and D.A.Drabold. Physica Stat. Solidi B, 246:1849, 2009.

[120] M.Boero, A.Bouzid, S.Le Roux, B.Ozdamar, and C.Massobrio. MOLECULAR DYNAMICS SIMULATIONS OF DISORDERED MATERIALS: FROM NETWORK GLASSES TO 
PHASE-CHANGE MEMORY ALLOYS.

[121] K.Prasai, P.Biswas, and D.A.Drabold. Sci. Rep.

[122] L.Pauling. The nature of the chemical bond and the structure of molecules and crystals; an introduction to modern structural chemistry. Cornell University Press, 1960.

[123] M.J.L.Sangster and M. Dixon. Adv. Phys., 25:247, 1976.

[124] A.J.Stone. Theory of Intermolecular Forces. Oxford University Press, 1996.

[125] Paul Peter Ewald. Die berchnung optischer und elektrostatischer gitterpotentiale. Ann. Phys., 64:253 - 287, 1921.

[126] M.Born and J.E.Mayer. Z. Phys., 75:1, 1932.

[127] L.Pauling. J.Am.Chem.Soc., 51:1010, 1929.

[128] L.V.Woodcock, C.A.Angell, and P.Cheeseman. J. Chem. Phys., 65:1565, 1976.

[129] B. W. H. van Beest, G. J. Kramer, and R. A. van Santen. Phys. Rev. Lett., 64:1955, 1990.

[130] P.Tangney and S.Scandolo. J. Chem. Phys., 117:8898, 2002.

[131] M.Wilson, P.A.Madden, M.Hemmati, and C.A.Angell. Phys. Rev. Lett., 77:4023, 1996.

[132] M.J.Sanders, C.R.A.Catlow, and J.V.Smith. J. Phys. Chem., 88:2796, 1984.

[133] C.R.A.Catlow and A.N.Cormack. Int. Rev. Phys. Chem., 6:227, 1987.

[134] E.Demiralp, T.Cagin, and W.A.Goddard III. Phys. Rev. Lett., 82:1708, 1999.

[135] D.Herzbach, K.Binder, and M.H.Mueser. J. Chem. Phys., 123:124711, 2005.

[136] B. G. Dick and A. W. Overhauser. Phys. Rev., 112:90, 1958.

[137] A.Aguado, L.Bernasconi, S.Jahn, and P.A.Madden. Faraday Discuss., 124:171, 2003.

[138] O.F.Mossotti. Mem. di mathem. e fisica in Modena, 24:49, 1850.

[139] R.Clausius. Die mechanische Waermetheorie, 2:62, 1879.

[140] B.K. Sharma and M. Wilson. Phys. Rev. B, 73:060201, 2006.

[141] B.K. Sharma and M. Wilson. J. Phys.: Condens. Matter, 20:244123, 2008.

[142] D.Marrocchelli, M.Salanne, P.A.Madden, C.Simon, and P.Turq. Mol. Phys., 107:443, 2009.

[143] D.Corradini, Y.Ishii, N.Ohtori, and M.Salanne. Model. and Sim. in Mat. Sci. and Eng., 23:074005, 2015.

[144] R.J.Heaton, R.Brookes, P.A.Madden, M.Salanne, C.Simon, and P.Turq. J. Phys. Chem. B, 110:11454, 2006.

[145] M.Salanne, C.Simon, P.Turq, R.J.Heaton, and P.A.Madden. J. Phys. Chem. B, 110:11461, 2006.

[146] M.Wilson and B.K.Sharma. J.Chem.Phys., 128:214507, 2008.

[147] M. Wilson. J. Chem. Phys., 124:124706, 2006.

[148] M. Wilson. J. Chem. Phys., 131:214507, 2009.

[149] M. Wilson and P. A. Madden. Phys. Rev. Lett., 72:3033, 1994.

[150] S.Erkoc. Phys. Rep., 278:79, 1997.

[151] F.H.Stillinger and T.A.Weber. Phys. Rev. B, 31:5262, 1985.

[152] V.Molinero, S.Sastry, and C.A.Angell. Phys. Rev. Lett., 97:075701, 2006.

[153] W.Hujo, B.S.Jabes, V.K.Rana, C.Chakravarty, and V.Molinero. J. Stat. Phys., 145:293, 2011.

[154] W.D.Luedtke and U.Landman. Phys. Rev. B, 37:4656, 1988.

[155] W.D.Luedtke and U.Landman. Phys. Rev. B, 40:1164, 1989.

[156] L.B.Skinner, C.J.Benmore, J.K.R.Weber, M.C.Wilding, S.K.Tumber, and J.B.Parise. PCCP, 15:08566, 2013.

[157] D.Machon, F.Meersman, M.C.Wilding, M.Wilson, and P.F.McMillan. Prog. Mat. Sci., 61:216, 2014.

[158] W. Kob and L. Berthier. Probing a liquid to glass transition in equilibrium. Phys. Rev. Lett., 110:245702, 2013.

[159] D.Daisenberger, M.Wilson, P.F.McMillan, R.Quesada Cabrera, M.C.Wilding, and D.Machon. Phys. Rev. B, 75:224118, 2007.

[160] S. Nosé. J. Chem. Phys., 81:511, 1984.

[161] W. G. Hoover. Phys. Rev. A, 31:1695, 1985.

[162] A.Zeidler, K.Wezka, D.A.J.Whittaker, P.S.Salmon, S.Klotz, H.E.Fischer, M.C.Wilding, C.L.Bull, 
M.G.Tucker, and M.Wilson. Phys. Rev. Lett., 113:135501, 2014.

[163] Sears V F. Neutron News, 3:26, 1992.

[164] L.Stixrude and M.S.T.Bukowinski. Am. Min., 75:1159, 1990.

[165] L.P.Davila, M-J Caturla, A.Kubota, B.Sadigh, T.D.de la Rubia, J.F.Shackelford, S.H.Risbud, and S.H.Garofalini. Phys.Rev. Lett., 91:205501, 2003.

[166] L.Huang and J.Kieffer. Phys. Rev. B, 69:224203, 2004.

[167] L.Huang and J.Kieffer. Phys. Rev. B, 69:224204, 2004.

[168] L.Huang, L.Duffrene, and J.Kieffer. J. Non-Cryst. Solids., 349:1, 2004.

[169] Liang Y, Miranda C R, and Scandolo S. Phys. Rev. B, 75:024205, 2007.

[170] A.C.Wright, R.N.Sinclair, and A.J.Leadbetter. J. Non-Cryst. Solids, 71:295, 1985.

[171] C.Massobrio and A.Pasquarello. Phys.Rev.B, 75:014206, 2007.

[172] R.Sharma, S.N.Chakraborty, and C.Chakravarty. J. Chem. Phys., 125:204501, 2006.

[173] P.Vashishta, R.K.Kalia, and I.Ebbsjo. Phys. Rev. B, 39:6034, 1989.

[174] P.Vashishta, R.K.Kalia, G.A.Antonio, and I.Ebbsjo. Phys. Rev. Lett., 62:1651, 1989.

[175] P.Vashishta, R.K.Kalia, J.P.Rino, and I.Ebbsjo. Phys. Rev. B, 41:12197, 1990.

[176] H.Iyetomi, P.Vashishta, and R.K.Kalia. Phys. Rev. B, 43:1726, 1991.

[177] D.Stauffer and A.Aharony. Introduction to percolation theory. Taylor and Francis, 1992.

[178] M.Wilson, P.A.Madden, N.N.Medvedev, A.Geiger, and A.Appelhagen. J.Chem. Soc: Faraday Trans., 94:1221, 1998.

[179] M.Wilson and P.A.Madden. Phys. Rev. Lett., 80:532, 199.

[180] J.D.Martin, S.J.Goettler, N.Fosse, and L.Iton. Nature, 419:381, 2002.

[181] J. C. Wasse and P. S. Salmon. Physica B, 241:967, 1998.

[182] J. C. Wasse and P. S. Salmon. J. Phys.: Condens. Matter 11, 11:1381, 1999.

[183] J. C. Wasse and P. S. Salmon. J. Phys.: Condens. Matter, 11:2171, 1999.

[184] F.Hutchinson, A.J.Rowley, M.K.Walters, M.Wilson, P.A.Madden, J.C.Wasse, and P.S.Salmon. J. Chem. Phys., 111:2028, 1999.

[185] F.Hutchinson, M.Wilson, and P.A.Madden. Mol. Phys., 99:811, 2001.

[186] C.S.Santos, N.S.Murthy, G.A.Baker, and E.W.Castner. J. Chem. Phys., 134:121101, 2011.

[187] C. Hardacre, J. D. Holbrey, C. L. Mullan, T. G. A. Youngs, and D. T. Bowron. J. Chem. Phys., 133:074510, 2010.

[188] A. Triolo, O. Russina, H.-J. Bleif, and E. di Cola. J. Phys. Chem. B, 111:4641, 2007.

[189] A. Triolo, O. Russina, B. Fazio, G. B. Appetecchi, M. Carewska, and S. Passerini. J. Chem. Phys., 130:164521, 2009.

[190] O. Russina, A. Triolo, L. Gontrani, R. Caminiti, D. Xiao, Jr. L. G. Hines, R. A. Bartsch, E. L. Quitevis, N. Pleckhova, and K. R. Seddon. J. Phys.: Condens. Matter, 21:424121, 2009.

[191] M.C.Wilding, M.Wilson, O.L.G.Alderman, C.J.Benmore, J.K.R.Weber, J.B.Parise, A.Tamalonis, and L.Skinner. Low-dimensional network formation in molten sodium carbonate. Sci. Rep., 6:24415, 2016.

[192] M.C.Wilding. To be published, 2016.

[193] R.J.F. Leote de Carvalho and R.Evans. Mol. Phys., 83:619, 1994.

[194] S. Sastry. Nature, 409:164, 2001.

[195] K.Binder and W.Kob. Glassy Materials and Disordered Solids. World Scientific, Singapore, 2005.

[196] P.G.de Gennes. Physica, 25:825, 1959.

[197] P.F.McMillan, G.N.Greaves, M.Wilson, M.C.Wilding, and D.Daisenberger. Adv. Chem. Phys., 152:309, 2013.

[198] L.-G. Liu and W.A.Bassett. Elements, Oxides and Silicates: High Pressure Phases with Implications for the Earth's Interior. Clarendon, Oxford, 1987.

[199] E.G.Ponyatovsky and O.I.Barkalov. Mater. Sci. Rep., 8:147, 1992.

[200] E.Rapoport. J. Chem. Phys., 46:2891, 1967.

[201] P.H.Poole, T.Grande, C.A.Angell, and P.F.McMillan. Science, 275:322, 1997. 
[202] P.F.McMillan. J. Mater. Chem., 14:1506, 2004.

[203] M.Wilson and P.F.McMillan. Phys. Rev. Lett., 90:135703, 2003.

[204] D.R.Robinson and M.Wilson. J. Phys.: Condens. Matter, 25:155101, 2013.

[205] M.P.Grumbach and R.M.Martin. Phase diagram of carbon at high pressures and temperatures. Phys. Rev. B, 54:15730, 1996.

[206] X.Wang, S.Scandolo, and R.Car. Carbon phase diagram from ab initio molecular dynamics. Phys. Rev. Lett., 95:185601, 2005.

[207] A.A.Correa, S.A.Bonev, and G.Galli. Carbon under extreme conditions: Phase boundaries and electronic properties from first-principles theory. PNAS, 103:1204, 2006.

[208] J.Tersoff. Phys. Rev. B, 37:6991, 1988.

[209] D.R.McKenzie, D.Muller, and B.A.Pailthorpe. Compressive-stress-induced formation of thin-film tetrahedral amorphous-carbon. Phys. Rev. Lett., 67:773, 1991.

[210] I.I.Aksenov, V.A.Belous, V.G.Padalka, and V.M.Khoroshikh. Sov.J. Plasma Phys., 4:425, 1978.

[211] P.H.Gaskell, A.Saeed, P.Chieux, and D.R.McKenzie. Neutron-sscattering studies of the structure of highly tetrahedral amorphous diamond-like carbon. Phys. Rev. Lett., 67:1286, 1991.

[212] R.Lossy, D.L.Pappas, R.A.Roy, J.J.Cuomo, and V.M.Sura. Filtered arc deposition of amorphous diamond. Appl. Phys. Lett., 61:171, 1992.

[213] J.Schwan, S.Ulrich, T.Theel, H.Roth, H.Ehrhardt, P.Becker, and S.R.P.Silva. Stress-induced formation of high-density amorphous carbon thin films. J. Appl. Phys., 82:6024, 1997.

[214] K.Ogata, Y.Andoh, and E.Kamijo. Crystallization of carbon-films by ion-beam assist technology. Nucl. Inst. Methods in Phys. Res., B33:685, 1988.

[215] P.J.Fallon, V.S.Veerasamy, C.A.Davis, J.Robertson, G.A.J.Amaratunga, W.I.Milne, and J.Koskinen. Properties of filtered-ion-beam-deposited diamond-like carbon as a function of ion energy. Phys. Rev. B, 48:4777, 1993.

[216] N.A.Marks, N.C.Cooper, D.R.McKenzie, D.G.McCulloch, P.Bath, and S.P.Russo. Comparison of density-functional, tight-binding, and empirical methods for the simulation of amorphous carbon. Phys. Rev. B, 65:075411, 2002.

[217] N.A.Marks. Modelling diamond-like carbon with the environment-dependent interaction potential. J. Phys.: Condens. Matter, 14:2901, 2002.

[218] G.Opletal, T.Petersen, B.O'Malley, I.Snook, D.G.McCulloch, N.A.Marks, and I.Yarovsky. Hybrid approach for generating realistic amorphous carbon structure using metropolis and reverse monte carlo. Molecular Simulation., 28:927, 2002.

[219] A.R.Ubbelohde. The Molten State of Matter. John Wiley \& Sons, 1978.

[220] N.A.Gokcen, E.T.Chang, T.M.Poston, and D.J.Spencer. Determination of graphite liquid-vapor triple point by laser-heating. High Temp. Sci., 8:81, 1976.

[221] C.T.Moynihan and C.A.Angell. J. Non-Cryst. Solids, 274:131, 2000.

[222] L.I.Aptekar. Sov. Phys. Dokl., 24:993, 1979.

[223] S. Sastry and C.A.Angell. Nature Materials, 2:739, 2003.

[224] S.K.Deb, M.C.Wilding, M.Somayazulu, and P.F.McMillan. Nature, 414:528, 2001.

[225] P.F.McMillan, M.Wilson, D.Daisenberger, and D.Machon. Nature Mater., 4:680, 2005.

[226] B.K.Sharma and M.Wilson. J.Phys.: Condens. Matter, 20:244123, 2008.

[227] V.V.Brazhkin, A.G.Lyapin, S.V.Popova, Y.Katayama, H.Saitoh, and W.Utsumi. J. Phys.: Condens. Matter, 19:246104, 2007.

[228] I.Saika-Voivod, F.Sciortino, and P.H.Poole. Phys. Rev. E, 63:011202, 2000.

[229] E.G.Ponyatovsky. J. Phys.: Condens. Matter, 15:6123, 2003.

[230] S.Straessler and C.Kittel. Phys. Rev., page 758, 1965.

[231] O.Kubaschewski, C.B.Alcock, and P.J.Spencer. Materials Thermochemistry. Pergamon, 1993.

[232] C.A.Angell, E.Williams, K.J.Rao, and J.C.Tucker. J. Phys. Chem., 81:238, 1977.

[233] C.A.Angell. Ann. Rev. Phys. Chem., 34:593, 1983.

[234] S.Sastry, F.Sciortino, and H.E.Stanley. chem. Phys. Lett., 207:275, 1993.

[235] P.G.Debenedetti. J.Phys.: Condes. Matter, 15:R1669, 2003. 
[236] P.H.Poole, F.Sciortino, and U.Essman. Nature, 360:6402, 1992.

[237] S.Sastry, P.G.Debenedetti, F.Sciortino, and H.E.Stanley. Phys. Rev. E, 53:6144, 1996.

[238] J.R.Errington and P.G.Debenedetti. Nature, 409:6818, 2001.

[239] M.S.Shell, P.G.Debenedetti, and A.Z.Panagiotopoulos. Phys. Rev. E, 66:011202, 2002.

[240] I.Saika-Voivod, F.Sciortino F, and P.H.Poole. Phys. Rev. E, 63:011202, 2001.

[241] A.Mudi and C.Chakravarty. J. Phys. Chem. B, 108:19607, 2004.

[242] A.Mudi and C.Chakravarty. J. Phys. Chem. B, 110:4502, 2006.

[243] A.Mudi and C.Chakravarty. J. Phys. Chem. B, 110:8422, 2006.

[244] A.Mudi, C.Chakravarty, and R.Ramaswamy. J. Chem. Phys., 122:104507, 2006.

[245] A.Mudi, C.Chakravarty, and R.Ramaswamy. J. Chem. Phys., 124:069902, 2006.

[246] A.Mudi, C.Chakravarty, and E.Milotti. J. Chem. Phys., 125:074508, 2006.

[247] R.Sharma, A.Mudi, and C.Chakravarty. J. Chem. Phys., 125:044705, 2006.

[248] M.Agarwal, R.Sharma, and C.Chakravarty. J. Chem. Phys., 127:164502, 2007.

[249] M.Agarwal and C.Chakravarty. J. Phys. Chem., 111:13294, 2007.

[250] B.S.Jabes, M.Agarwal, and C.Chakravarty. J. Chem. Phys., 132:234507, 2010.

[251] M.R.Sadr-Lahijany, A.Scala, S.V.Buldyrev, and H.E.Stanley. Phys. Rev. Lett., 81:4895, 1998.

[252] N.B.Wilding and J.E.Magee. Phys. Rev. E, 66:031509, 2002.

[253] H.M.Gibson and N.B.Wilding. Phys. Rev. E, 73:061507, 2006.

[254] G.Ferlat, T.Charpentier, A.P.Seitsonen, A.Takada, M.Lazzeri, L.Cormier, G.Calas, and F.Mauri. Phys. Rev. Lett., 101:065504, 2008.

[255] J.Swenson and L.Borjesson. Phys. Rev. B, 55:11138, 1997.

[256] P.A.V.Johnson, A.C.Wright, and R.N.Sinclair. J Non-Cryst. Solids, 50:281, 1982.

[257] A.C.Hannon, D.I.Grimley, R.A.Hulme, A.C.Wright, and R.N.Sinclair. J Non-Cryst. Solids, 177:299, 1994.

[258] A.Zeidler, K.Wezka, D.A.J.Whittaker, P.S.Salmon, A.Baroni, S.Klotz, H.E.Fischer, M.C.Wilding, C.L.Bull, and M.G.Tucker. Phys. Rev. B, 90:024206, 2014.

[259] O.J.G.Alderman, G.Ferlat, A.Baroni, M.Salanne, M.Micoulaut, C.J.Benmore, A.Lin, A.Tamalonis, and J.K.R.Weber. J. Phys.: Condens. Mat., 27:455104, 2015.

[260] C.Gomez-Navarro, J.C.Meyer, R.S.Sundaram, A.Chuvilin, S.Kurasch, M.Burghard, K.Kern, and U.Kaiser. Nano Lett., 10:1144, 2010.

[261] D.L.Weaire and N.Rivier. Contemp.Phys., 25:59, 1984.

[262] S.Torquato and F.H.Stillinger. Phys. Rev. E, 68:041113, 2003.

[263] M.Hejna, P.J.Steinhardt, and S.Torquato. Phys. Rev. B, 87:245204, 2013.

[264] A.K.Geim. Science, 324:1530, 2009.

[265] A.K. Geim. Graphene: Status and Prospects. Science, 324:1530-1534, June 2009.

[266] D.R.Robinson. Part II Thesis, University of Oxford. 2012.

[267] J.Long. Part II Thesis, University of Oxford. 2013.

[268] C.Leandri, H.Oughaddou, B.Aufray, J.M.Gay, G. Le Lay, A.Ranguis, and Y.Garreau. Surf. Sci., 601:262, 2007.

[269] A.Kara, C.Leandri, M.E.Davila, P. De Padova, B.Ealet, H.Oughaddou, B.Aufray, and G. Le Lay. J. Supercond. Nov. Magn., 22:259, 2009.

[270] B.Aufray, A.Kara, S.Vizzini, H.Oughaddou, C.Leandri, B.Ealet, and G. Le Lay. Appl. Phys. Lett., 96:183102, 2010.

[271] P.De Padova, C.Quaresima, C.Ottaviani, P.M.Sheverdyaeva, P.Moras, C.Carbone, D.Topwal, B.Olivieri, A.Kara, and H.Oughaddou. Appl. Phys. Lett., 96:261905, 2010.

[272] M.Houssa, G.Pourtois, M.M.Heyns, V.V.Afanasev, and A.Stesmans. J. Electrochem. Soc., 158:H107, 2011.

[273] M.Houssa, G.Pourtois V.V.Afanasev, and A.Stesmans. Appl. Phys. Lett.., 97:112106, 2010.

[274] S.Cahangirov, M.Topsakal, E.Akturk, H.Sahin, and S.Ciraci. Phys. Rev. Lett., 102:236804, 2009.

[275] F.H.Stillinger. J. Chem. Phys., 65:3968, 1976.

[276] F.H.Stillinger and T.A.Weber. J. Chem. Phys., 68:3837, 1978. 
[277] F.H.Stillinger and T.A.Weber. J. Chem. Phys., 70:4879, 1979.

[278] F.H.Stillinger. Phys. Rev. B, 20:299, 1979.

[279] S.Prestipino, F.Saija, and P.V.Giaquinta. Phys. Rev. E, 71:050102(R), 2005.

[280] S.Prestipino, F.Saija, and P.V.Giaquinta. Phys. Rev. Lett., 106:235701, 2011.

[281] A.Lang, C.N.Likos, M.Watzlawek, and H.Löwen. J. Phys.: Condens. Mat., 12:5087, 2000.

[282] H.S.M.Coexter. Regular Polytopes. Dover, 1973.

[283] F.van Swol, L.V.Woodcock, and J.N.Cape. J. Chem. Phys., 73:913, 1980.

[284] D.A.Aboav. Metallography, 3:383, 1970.

[285] D.Weaire. Metallography, 7:157, 1974.

[286] S.N.Chui. Materials Characterization, 34:149, 1995.

[287] F.T.Lewis. Anatomical Record, 38:341, June 1928.

[288] F.T.Lewis. Anat. Rec., 50:235, 1931.

[289] J.K.Mason, R.Ehrenborg, and E.A.Lazar. J. Phys A: Math. Theor., 45:065001, 2012.

[290] D.Weaire and S.Hutzler. The Physics of Foams. Oxford University Press, 2001.

[291] A.Kumar, M.Wilson, and M.F.Thorpe. Amorphous graphene: a realization of zachariasen's glass. J. Phys.: Condens. Mat., 24:485003, 2012.

[292] T.Aste and D.Sherrington. Glass transition in self-organizing cellular patterns. J. Phys. A: Math. Gen., 32:7049, 1999.

[293] A.Sartbaeva, S.A.Wells, M.M.J.Treacy, and M.F.Thorpe. The flexibility window in zeolites. Nature Materials, 5:962, 2006.

[294] Q Williams and E Knittle. Structural complexity in carbonatite liquid at high pressures. Geophys. Res. Lett., 30:1022, 2003.

[295] M J Genge, G D Price, and A P Jones. Molecular-dynamics simulations of caco3 melts to mantle pressures and temperatures - implications for carbonatite magmas. Earth Plan. Sci. Lett., 131:225, 1995.

[296] W.G.Hoover and F.H.Ree. J. Chem. Phys., 49:3610, 1968.

[297] W.G.Hoover, M.Ross, K.W.Johnson, D.Henderson, A.Barker, and E.C.Brown. J. Chem. Phys., 52:4931, 1970.

[298] J-P.Hansen and L.Verlet. Phys. Rev., 184:151, 1969.

[299] R.W.Hockney and T.R.Brown. J. Phys. C, 8:1813, 1975.

[300] M.Wilson. in Molecular Dynamics Dimulations of Disordered Materials. Springer, 2015. 
Structure and dynamics in network-forming materials.

\begin{tabular}{|c|c|c|c|c|}
\hline Model/System & $\begin{array}{r}\Delta S(30 \\
/ \mathrm{JK}\end{array}$ & $\begin{array}{l}S(2 \mathrm{~d}) \\
1^{-1}\end{array}$ & $\Delta S(3 \mathrm{~d}) / \Delta S(2 \mathrm{~d})$ & Reference \\
\hline Hard spheres & 9.6 & 3.4 & 2.8 & $\overline{[296]}$ \\
\hline Soft spheres & 6.7 & 2.1 & 3.2 & {$[297,283]$} \\
\hline Lennard-Jones potential & 14.5 & 4.2 & 3.5 & {$[298]$} \\
\hline One component plasma & 6.8 & 1.7 & 4.0 & {$[298,299,283]$} \\
\hline Carbon & 29.4 & 7.5 & 4.0 & {$[300,204]$} \\
\hline Silicon & 27.1 & - & - & {$[219]$} \\
\hline Germanium & 24.8 & - & - & [219] \\
\hline
\end{tabular}

Table 1. Melting entropies for a selection of systems. The ratio of the values for carbon obtained in two- and three-dimensions (fourth column) is consisten with those obtained for the models listed. In addition, the entropy obtained in three-dimensions is consistent with the values for conformal $\mathrm{Si}$ and Ge.

\begin{tabular}{|c|c|c|c|c|c|c|}
\hline & $T_{m} / \mathrm{K}$ & $T_{g} / \mathrm{K}$ & $T_{c} / \mathrm{K}$ & $T_{c} / T_{m}$ & $T_{c} / T_{g}$ & $T_{g} / T_{m}$ \\
\hline $\mathrm{ZnCl}_{2}$ & 591 & 375 & 825 & 1.40 & 2.20 & 0.63 \\
$\mathrm{H}_{2} \mathrm{O}$ & 273 & 136 & 194 & 0.71 & 1.40 & 0.50 \\
$\mathrm{SiO}_{2}$ & 1990 & 1450 & 730 & 0.37 & 0.50 & 0.73 \\
$\mathrm{Si}$ & 1680 & $800-950$ & 1060 & 0.63 & $1.12-1.33$ & $0.74-0.90$ \\
$\mathrm{C}$ & 15500 & 11600 & - & - & - & 0.74 \\
\hline
\end{tabular}

Table 2. Transition temperatures for a range of network systems. Key: $T_{m}-$ melting temperature, $T_{c}$ - low $\longrightarrow$ high density polymorph critical temperature, $T_{g}$ glass transition temperature. 


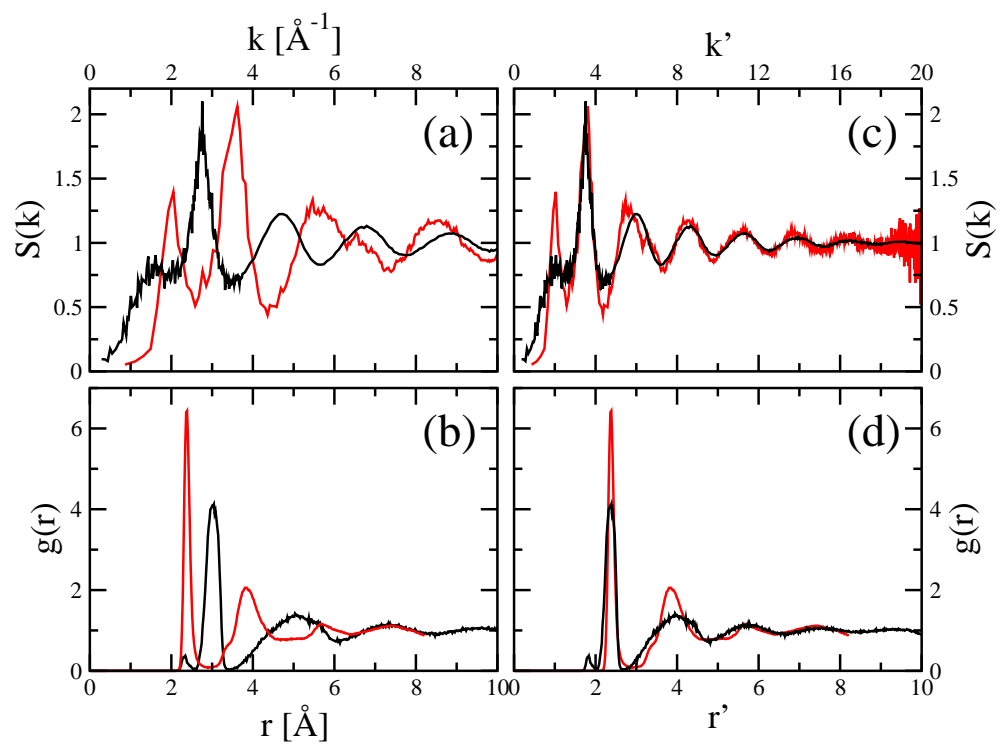

Figure 1. (a) Structure factors for a-Si (red line) and $\mathrm{SiO}_{2}$ (black line) obtained using the Sillinger -Weber [151, 159, 154, 155] and TS potentials [130] respectively. (b) Radial distribution functions for the same systems. (c) and (d) show the functions from (a) and (b) scaled in the ordinate axis by the Si-Si nearest-neighbour separation as described in the text.

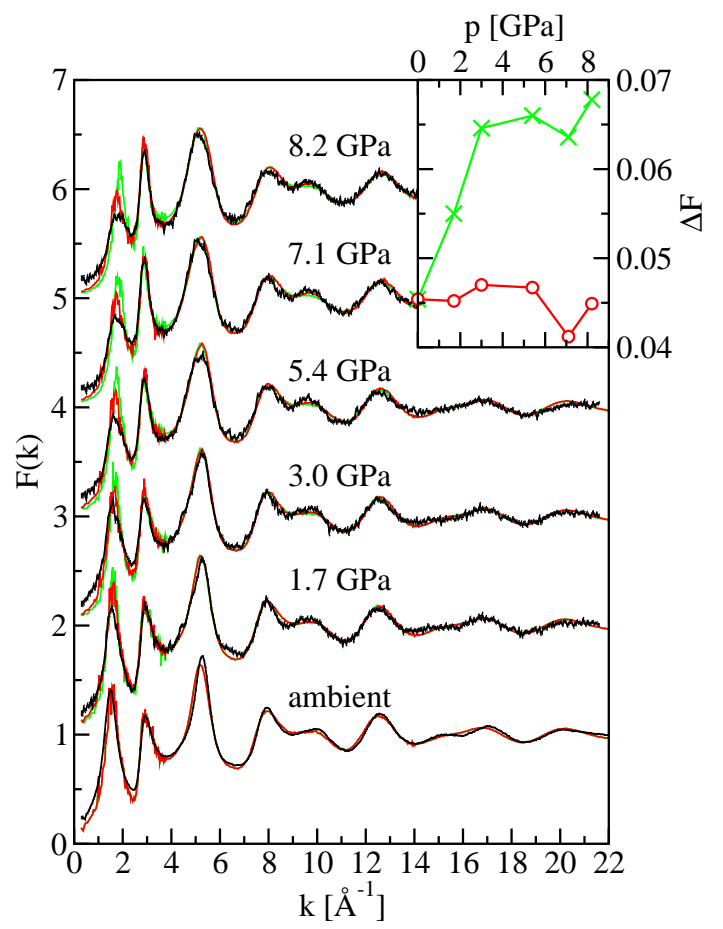

Figure 2. (Main panel) The black lines show the total scattering function, $F(k)$, for $\mathrm{SiO}_{2}$ obtained from neutron diffraction experiments [162]. The green and red lines show the corresponding results from simulation obtained using configurations from quenching and cold compression respectively. The pressures at which the experimental functions are obtained are shown next to the respective curves. Successive curves are offset along the abscissa. The inset shows the quality of fit (as described in the text) Key: $\times$ - liquid quench, $\circ$ - cold compressed. configurations respectively. 


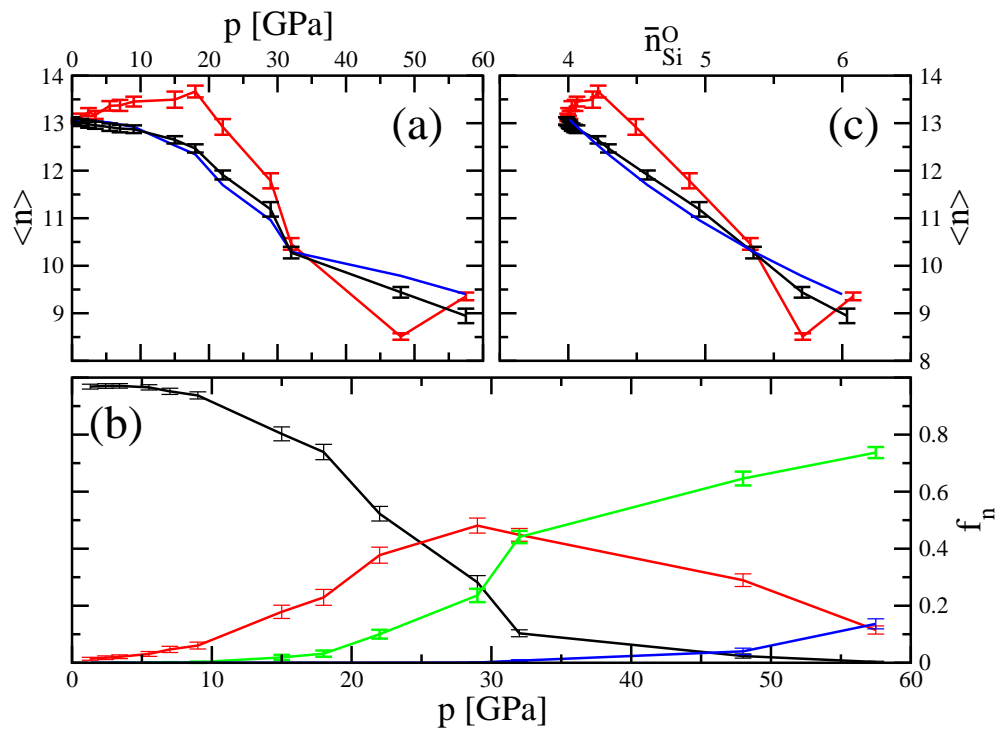

Figure 3. (a) Mean ring size, $\langle n\rangle, v$. pressure obtained for the glassy $\mathrm{SiO}_{2}$. Key: red line - quench, black line - cold compression. The behaviour predicted by the ring closure model (section 3.2.1) is shown as the blue line. (b) The fraction of sites with different coordination numbers for the cold compressed configurations. Key: black line - four-coordinate, red line - five-coordinate, green line - six-coordinate, blues line - seven-coordinate. (c) Showing the correlation between the mean ring size and mean Si-O nearest-neighbour coordination number, $\bar{n}_{\mathrm{Si}}^{\mathrm{O}}$.

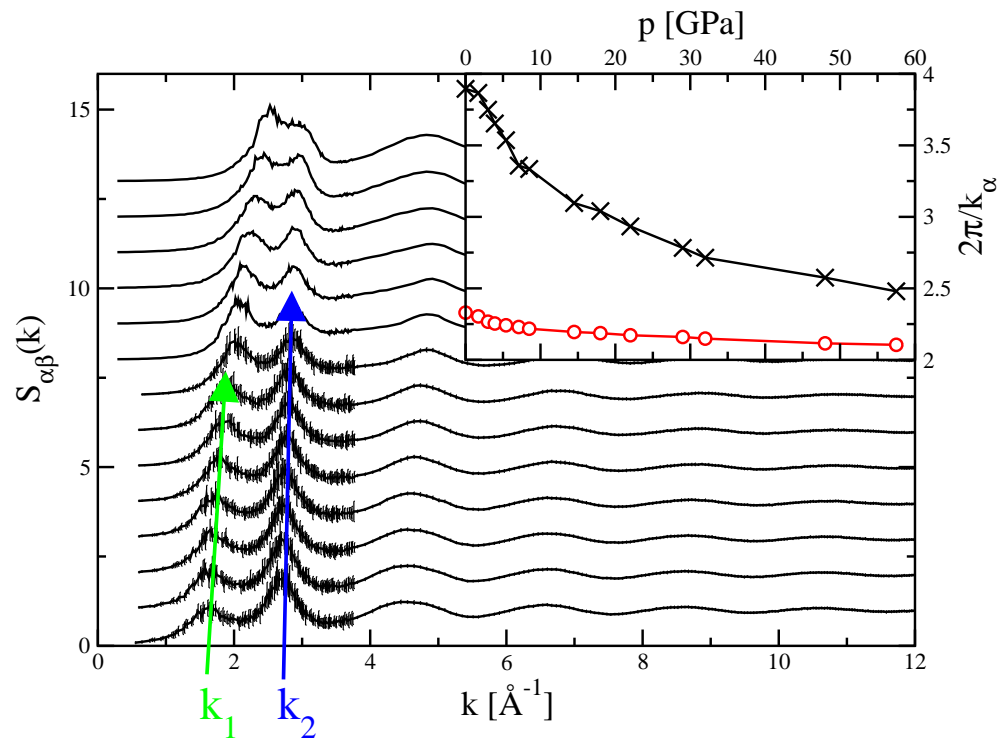

Figure 4. (Main panel) Partial structure factor, $S_{S i S i}(k)$, generated from $\mathrm{SiO}_{2}$ cold compression from ambient pressure. Successive pressure increases are offset along the ordinate axis for clarity. The inset shows the evolution of the length-scales associated with the first and second peaks (highlighted at $k_{1}$ and $k_{2}$ in the main panel, shown as $x$ and $\circ$ respectively) as a function of pressure. 

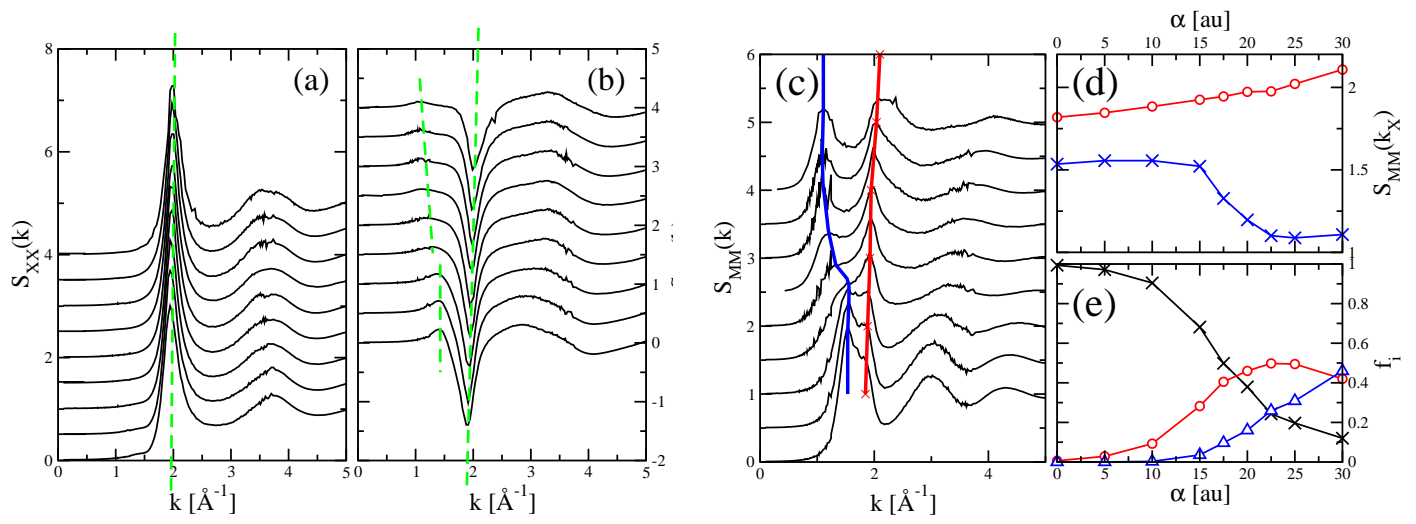

Figure 5. Ashcroft-Langreth partial structure factors for liquid $\mathrm{MX}_{2}$ (calculated along the $T=1000 \mathrm{~K}$ isotherm) as a function of anion polarizability, $\alpha$. The functions shown are (a) $S_{X X}(k)$, (b) $S_{M X}(k)$, (c) $S_{M M}(k)$. Successive functions are offset along the ordinate axis for clarity and, in each panel, correspond to (from bottom to top) $\alpha=0,5,10,15,17.5,20,22.5,25$ and 30au respectively. In (c) lines are superimposed to highlight the evolution of the first two peak positions (which evolve to form the principal and first-sharp diffraction peaks [PP and FSDP] at high $\alpha$ ). (d) The evolution of the positions of the PP and FSDP as a function of $\alpha$. (e) The evolution of the fractions of sites labelled "0", "1" and "2" ( $\times$, ० and $\triangle$ respectively) with $\alpha$.

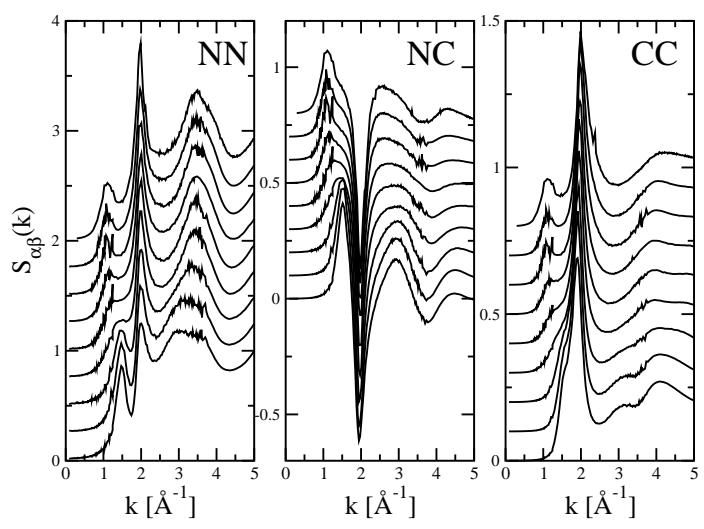

Figure 6. Bhatia-Thornton partial structure factors for liquid $\mathrm{MX}_{2}$ (calculated along the $T=1000 \mathrm{~K}$ isotherm) as a function of anion polarizability, $\alpha$. The functions shown are (from left to right) $S_{N N}(k), S_{N C}(k)$ and $S_{C C}(k)$. Successive functions are offset along the ordinate axis for clarity and, in each panel, correspond to (from bottom to top) $\alpha=0,5,10,15,17.5,20,22.5,25$ and 30au respectively. 

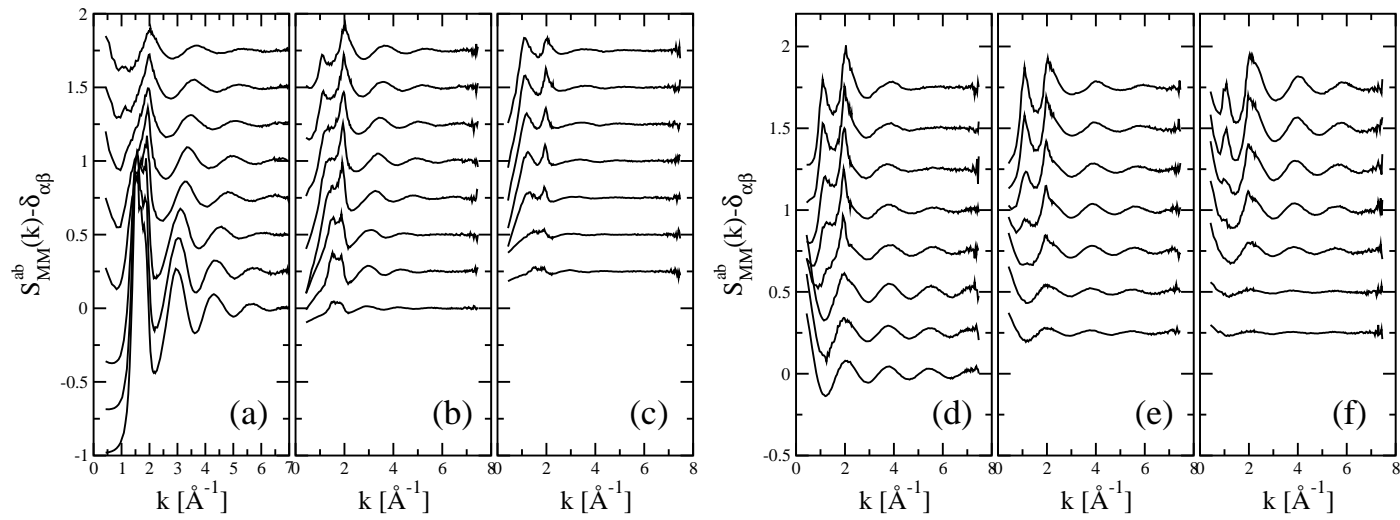

Figure 7. Six "coloured" cation-cation partial structure factors, $S_{M M}^{a b}(k)-\delta_{\alpha \beta}$, $\{a, b\}=\{0,1,2\}$ shown at different anion polarizabilies and calculated at $T=1000 \mathrm{~K}$. (a) $S_{M M}^{00}(k),(\mathrm{b}) S_{M M}^{01}(k),\left(\right.$ c) $S_{M M}^{02}(k),(\mathrm{d}) S_{M M}^{11}(k)$, (e) $S_{M M}^{12}(k)$ and (f) $S_{M M}^{22}(k)$. Key (from bottom to top): $\alpha=0,5,10,15,17.5,20,22.5$ and $25 \mathrm{au}$. For the functions with $b=2$ (those labelled 02,12 and 22 respectively) the $\alpha=0$ curve is absent, reflecting the lack of any sites labelled "2" at that limit. Successive curves are offset along the ordinate axis for clarity.
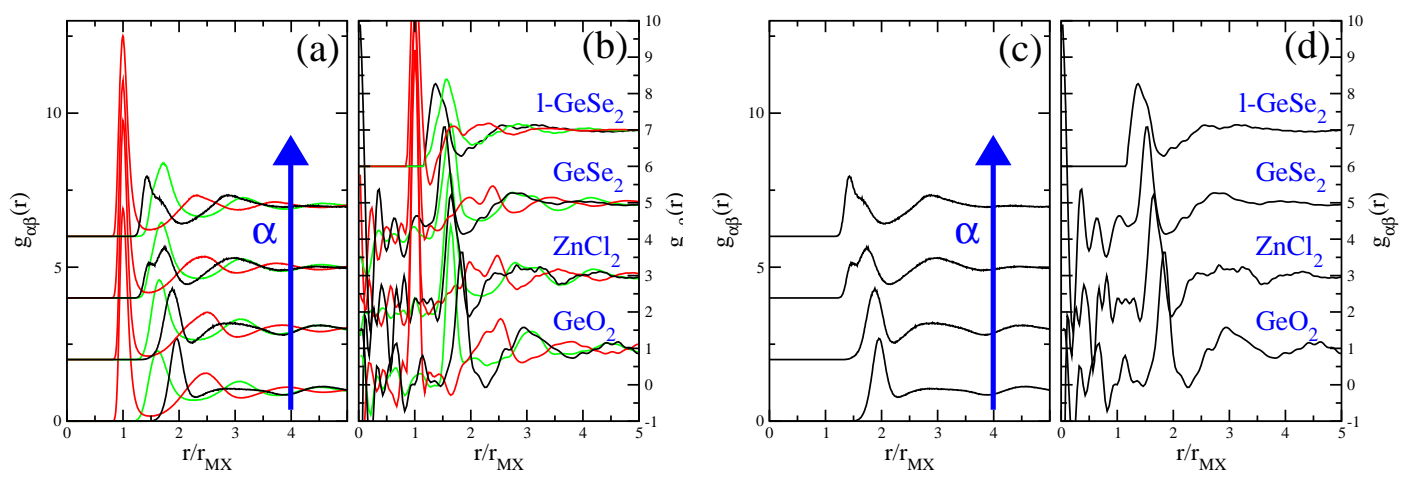

Figure 8. (a) Pair distribution functions as a function of anion polarizability, $\alpha$. Key: red - $g_{M X}(r)$, green - $g_{X X}(r)$, black - $g_{M M}(r)$. Successive functions are offset along the ordinate axis for clarity. The functions shown are for (from bottom to top) $\alpha=0$, 10, 20 and 30au respectively. (b) Pair distribution functions obtained experimentally using isotopic substitution for $\mathrm{GeO}_{2}[94,95], \mathrm{ZnCl}_{2}[44,92]$ and $\mathrm{GeSe}_{2}$ in the liquid $[27,93]$ and glassy $[28,93]$ states. Successive functions are offset along the ordinate axis for clarity. The functions are coloured as in panel (a). Panels (c) and (d) show the cation-cation functions only from panels (a) and (b). 


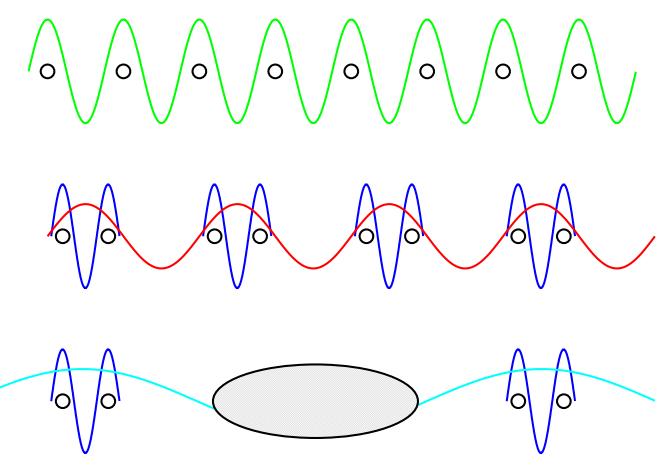

Figure 9. Schematic to demonstrate the development of ordering on multiple lengthscales in a simple chain of atoms. The upper chain consists of evenly-spaced atoms and so leads to a single (charge-ordered) length-scale. As pairs of nearest-neighbour atoms are brought together in the central chain two length-scales (ones shorter and one longer than the original) emerge. In the lower panel a large templating molecule is introduced which generates an even longer length-scale. 

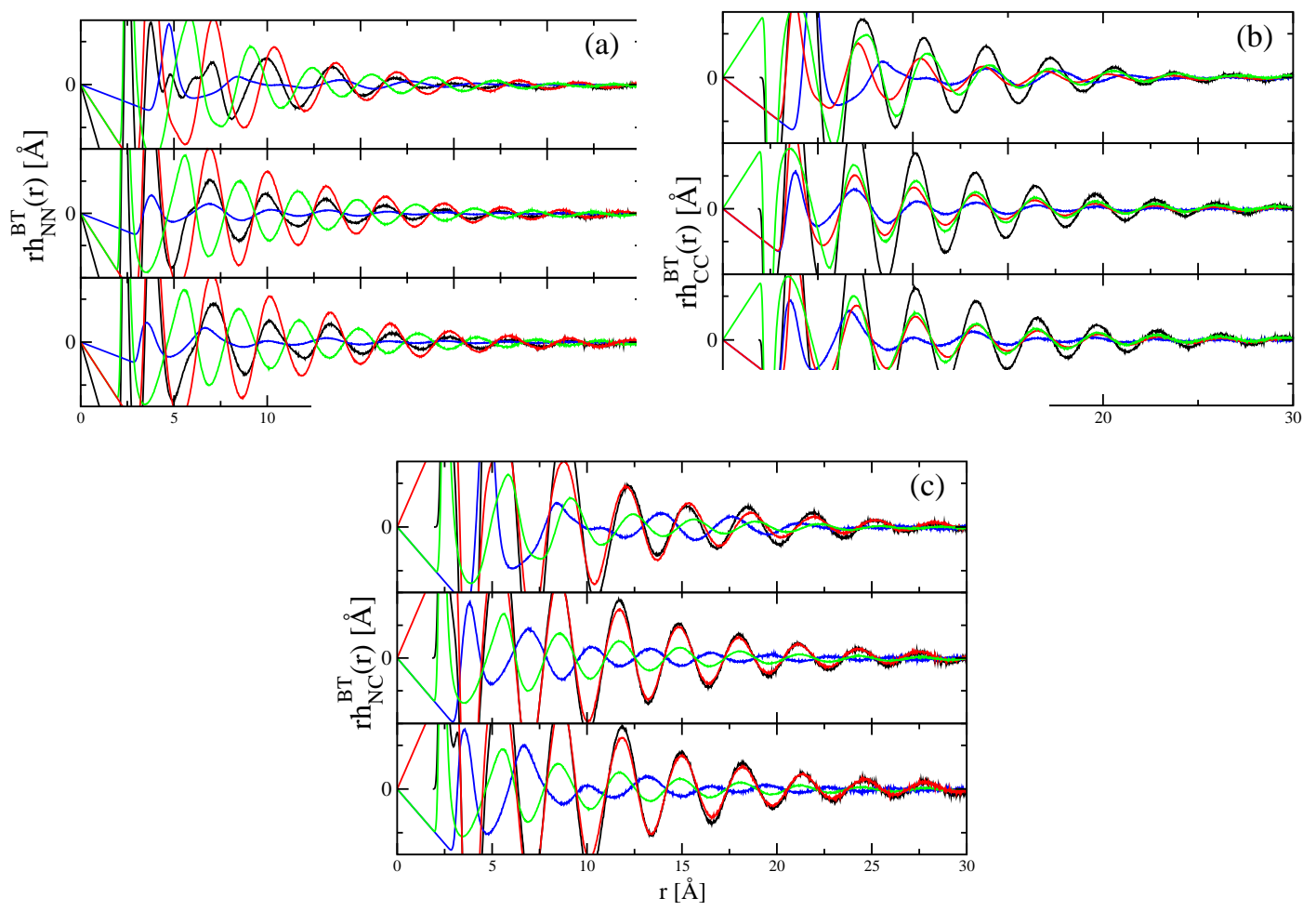

Figure 10. In all panels the black lines show the Bhatia-Thornton total pair distribution functions, $r h_{I J}^{B T}(k)(\{I, J\}=\{N, C\})$. (a) $h_{N N}^{B T}(k)$, (b) $h_{C C}^{B T}(k)$, (c) $h_{N C}^{B T}(k)$. In each the rigid ion (zero polarizability), intermediate and high polarizability model results are shown in the top, central and bottom panels respectively. Each BT function is shown with the underlying weighted atom-atom distribution functions. Key: red lines - MX, green lines - XX, blue lines - MM.
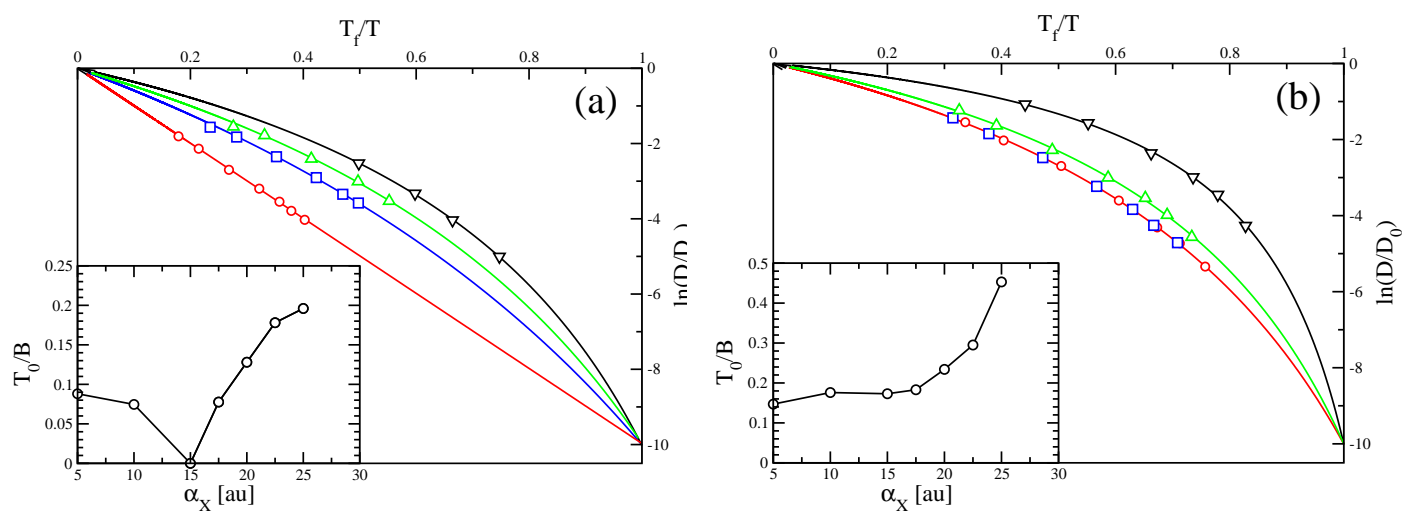

Figure 11. Cation diffusion coefficients, $D$, as a function of $T$ calculated over a range of anion polarizabilites, $\alpha$, and shows as $\ln D v .1 / T$ in order to highlight the respective curvatures (which relate to the liquid fragility). The diffusion coefficients are generated at two densities (shown in (a) and (b), panel (a) corresponding to the higher density). The diffusion coefficients are fitted to a VTF function as described in the text. In both cases the insets show the VTF fitting parameters, $T_{0} / B$, as a function of $\alpha$, highlighting the increase in system fragility as $\alpha$ increases. 

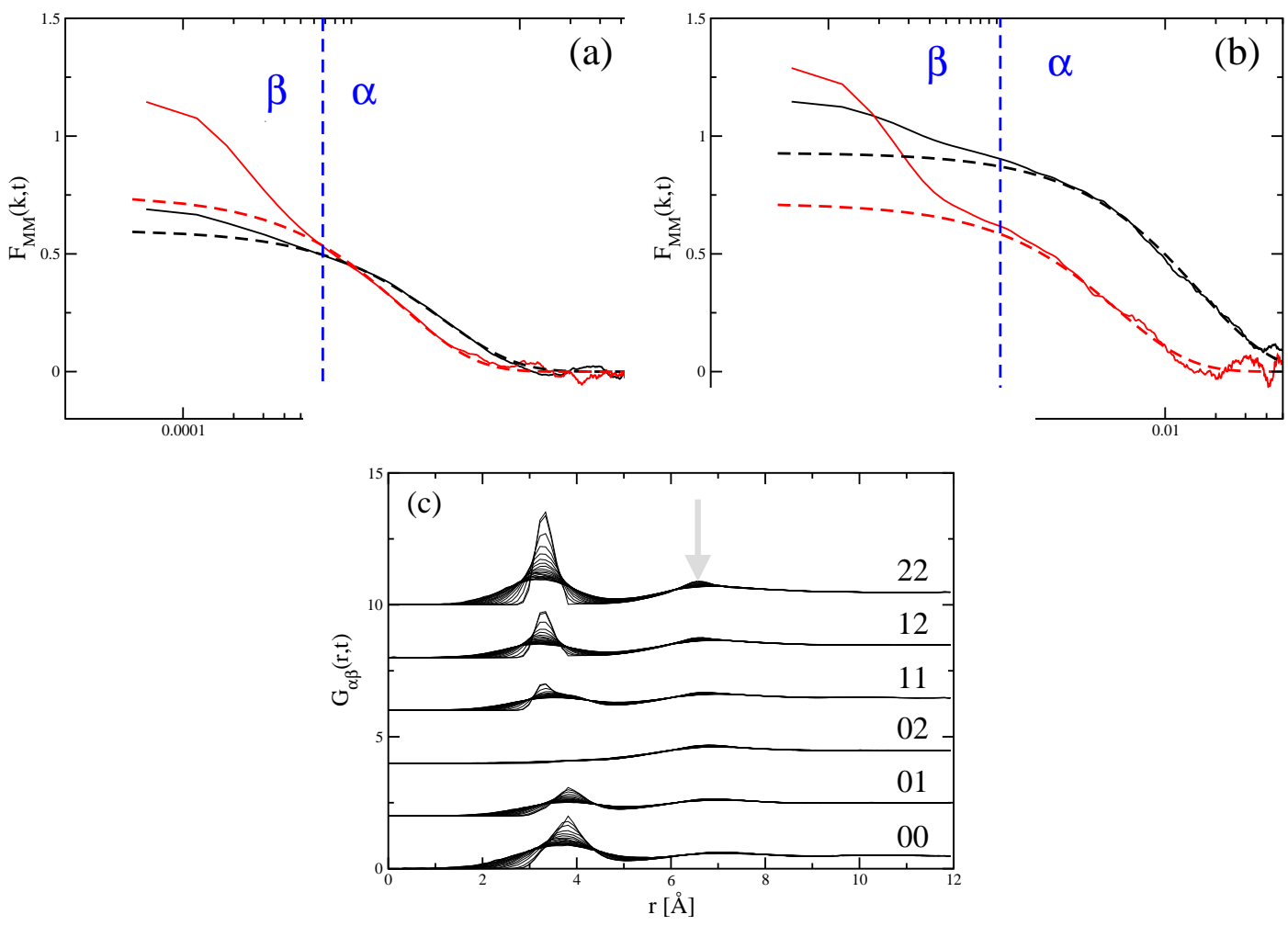

Figure 12. Intermediate scattering functions calculated for the cations, $F_{M M}(k, t)$, at $T=800 \mathrm{~K}$ for (a) $\alpha=15 \mathrm{au}$ and (b) $\alpha=25 \mathrm{au}$. In both cases the functions are shown for scattering angles which correspond to the positions of the respective first-sharp diffraction peaks (black lines) and principal peaks (red lines). The approximate $\alpha-$ and $\beta$ - relaxation regimes are indicated. The dashed lines show the results of fitting the $\alpha$-relaxation regime to stretched exponential functions. (c) Distinct van Hove spatial correlation functions $G_{\alpha \beta}\left(\mathbf{r}^{\prime}, \mathbf{r}, t\right)$ obtained from the local cation environments $(\alpha \beta=\{00,01,02,11,12,22\}$ for $\alpha=22.5 \mathrm{au}$. Successive functions are offset along the ordinate axis for clarity. The arrow highlights the emergence of the length-scale which corresponds to the first-sharp diffraction peak position in $S_{M M}(k)$.

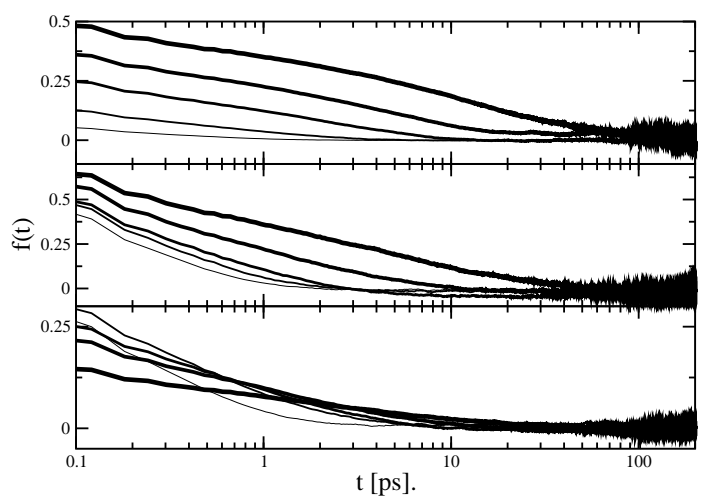

Figure 13. Time correlation functions constructed from the cation sites "coloured" by their connectivity as described in the text for $\alpha=22.5 \mathrm{au}$. The upper, central and lower panels show the respective decays of the functions for sites labelled ' 2 ', ' 1 ' and ' 0 ' determined at different temperatures of $T=1500,1200,1000,900$ and $800 \mathrm{~K}$ (thinnest to thickest lines). 

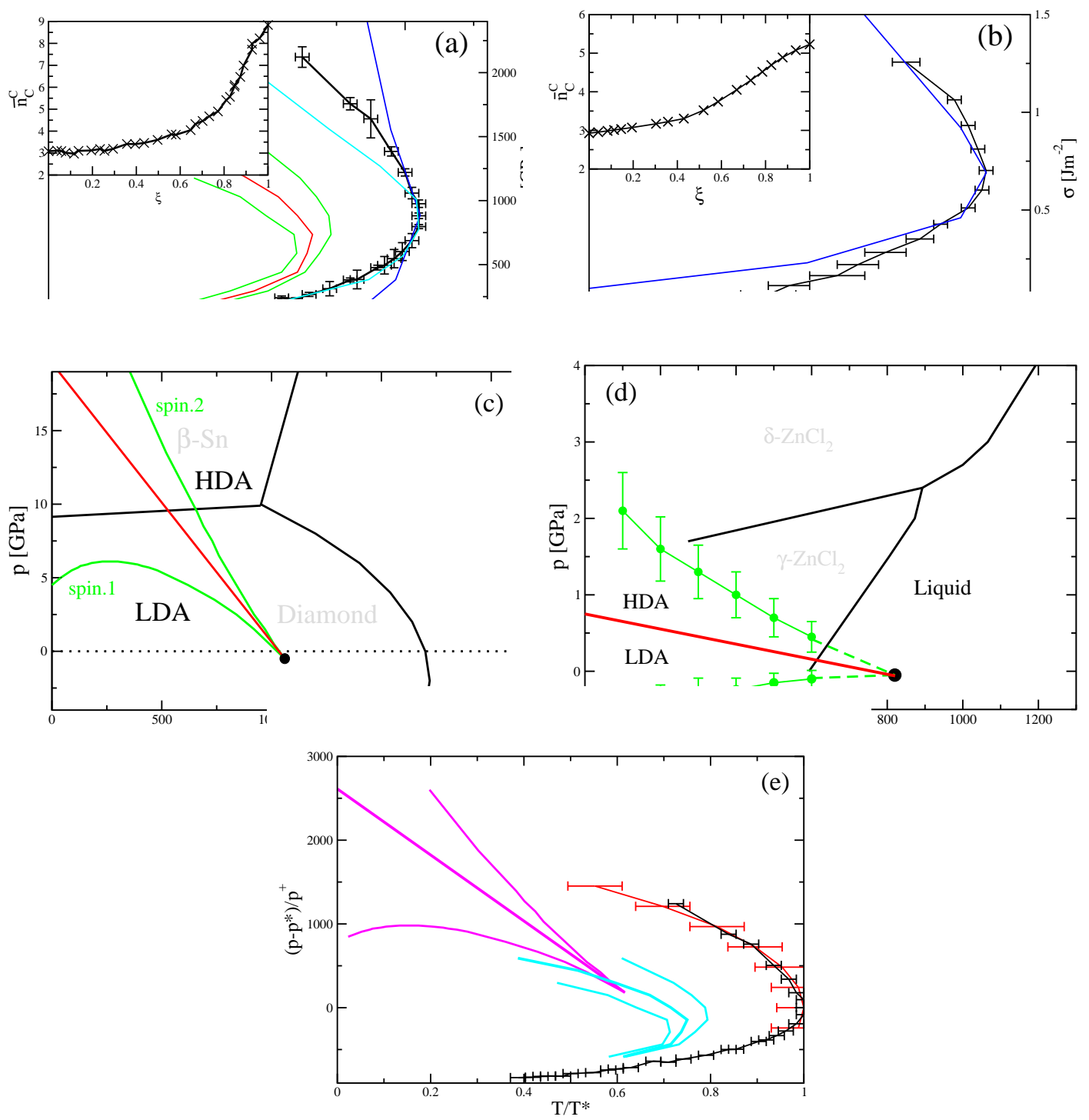

Figure 14. Phase diagrams highlighting key coexistence phenomena for a range of network systems. (a) Three-dimensional liquid/diamond crystal coexistence for carbon, (b) Two-dimensional liquid/hexagonal net crystal coexistence for carbon, (c) Three-dimensional LDA/HDA coexistence for silicon, (d) Three-dimensional LDA/HDA coexistence for $\mathrm{ZnCl}_{2}$, (e) Scaled phase diagrams for carbon and silicon. In panels (a) and (b) the coexistence curves are shown as black lines and the respective insets show how the mean carbon coordination numbers evolve on moving around the coexistence curve. The blue lines show the coexistence curves scaled from gaussian core models [279, 280]. In panel (a) the red and green lines show the liquid/amorphous coexistence curves and spinodals. In panels (c) and (d) the coexistence curves are shown as red lines with the corresponding spinodals as a pair of green lines which emanate from a critical point. The black lines indicate the predicted liquid/crystal coexistence curves. In panel (e) the black and red lines show the reduced liquid/crystal coexistence curves for carbon and silicon respectively. The magenta and light blue lines show the respective Si LDA/HDA and carbon liquid/amorphous coexistence curves and associated spinodals scaled in the same manner (thick and thin lines respectively). 

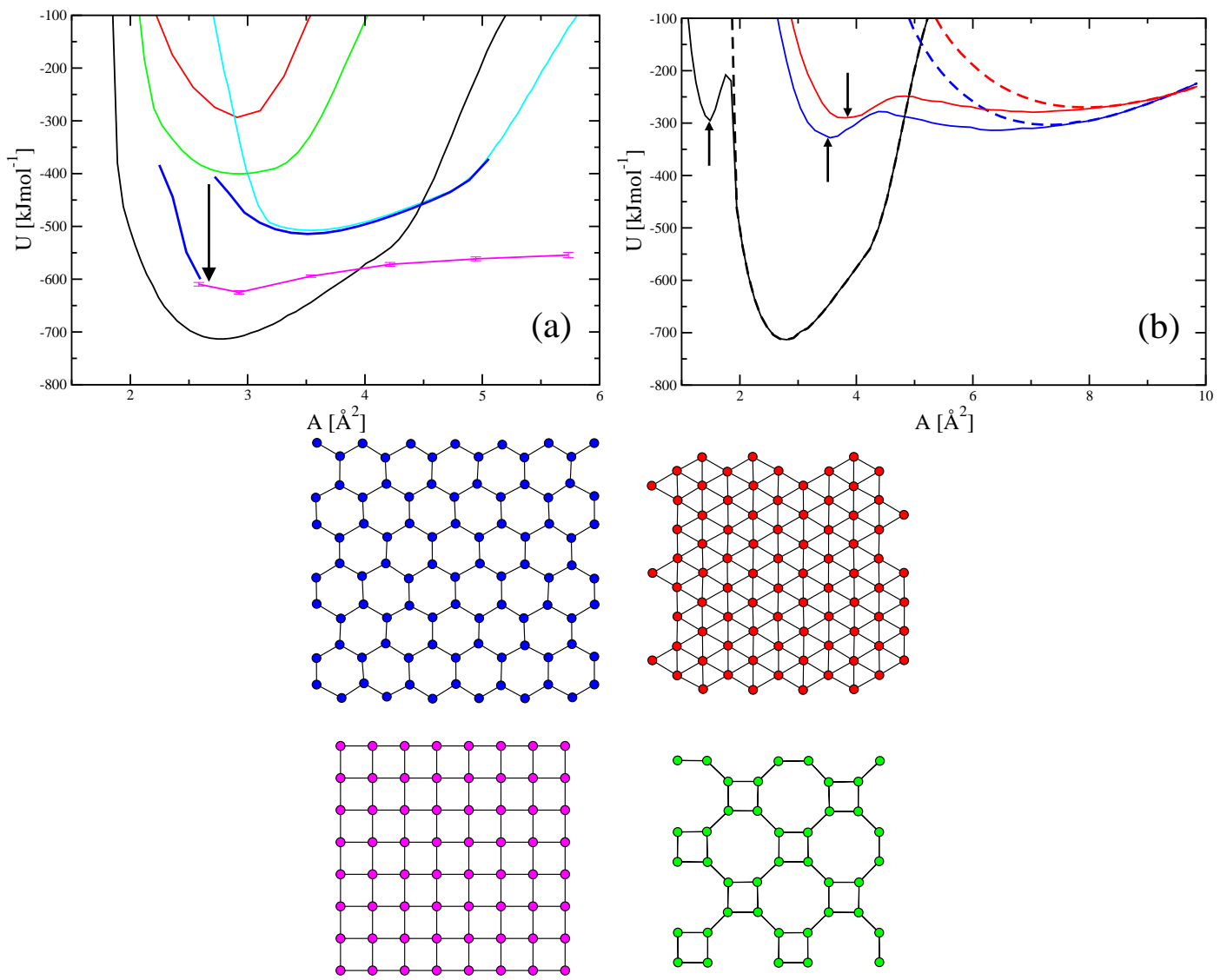

Figure 15. (a) The energies for four two-dimensional crystals determined over a range of densities (areas). The black, red, green and light blue lines correspond to the energies of the hexagonal net, close-packed lattice, square net and 4/8 crystal respectively. The magenta line shows the energies of a-G configurations. The blue line shows the effect of relaxing the $4 / 8$ crystal with the arrow highlighting the amorphisation transition observed for $A \lesssim 2.6 \AA^{2}$. (b) The energies of puckered (solid lines) and planar (dashed lines) forms of the hexagonal net. Key: black lines - carbon, blue lines - silicon, red lines - germanium. In all three cases the arrows highlight the energy minima of the puckered structure. (c) Molecular graphics images of the four two-dimensional crystal structures. Key: blue - hexagonal net, red - close-packed lattice, magenta - square net, green $-4 / 8$. 

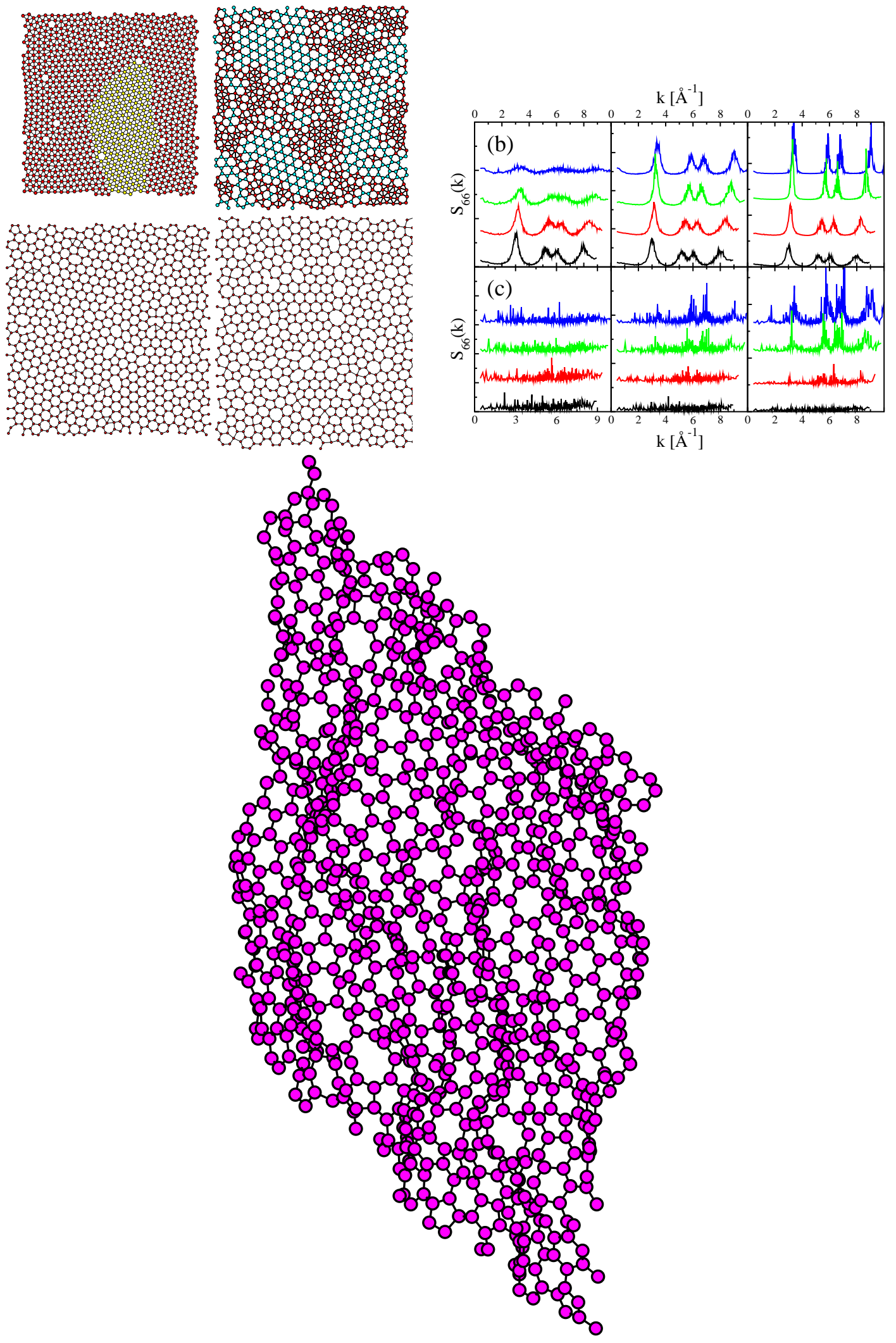

Figure 16. (a) Molecular graphics images of four configurations obtained at densities. Bottom right - $A \sim 2.57 \AA^{2}$, bottom left - $A \sim 2.16 \AA^{2}$, top right - $A \sim 1.95 \AA^{2}$, top left $A \sim 1.58 \AA^{2}$. At the highest density (top left) distinct nanocrystallites are observed and one is highlighted. At $A \sim 1.95 \AA^{2}$ (top right) domains of close-packed and hexagonal nets are formed. At $A \sim 2.16 \AA^{2}$ (bottom left) nanocrystalline domains are evident whilst at $A \sim 2.57 \AA^{2}$ (bottom right) the structure appears more amorphous. (b) Ring structure factors, $S_{66}(k)$ and (c) their second moments, $M_{66}^{(2)}(k)$, defined as described in the text. Key: black lines - $A \sim 2.57 \AA^{2}$, red lines - $A \sim 2.35 \AA^{2}$, green lines $A \sim 2.16 \AA^{2}$, blue lines - $A \sim 1.95 \AA^{2}$. The panels show the results using different cooling rates from the most rapid cooling (left panel) to slowest cooling (right panel). 


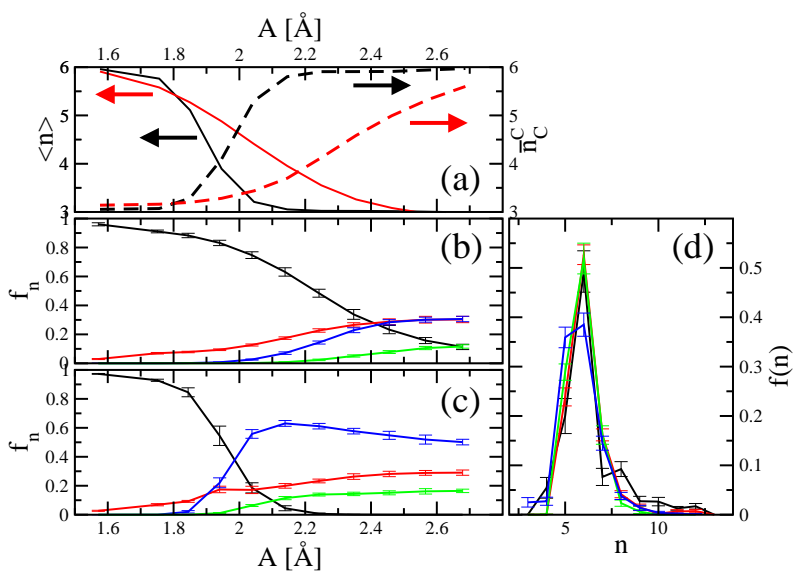

Figure 17. (a)-(c). The evolution of key structural properties for the liquid and amorphous graphene networks. Key: (a) solid lines - mean coordination number, $\bar{n}_{\mathrm{C}}^{\mathrm{C}}$, dashed lines - mean ring size, $\langle n\rangle$, black lines - amorphous, red lines - liquid. (b) and (c) show the fractions of rings of a given size for the (b) liquid and (c) amorphous configurations. Key: black line - three-membered rings, red line - four-membered rings, blue line - five-membered rings, green line - six-membered rings. (d) Ring size distributions obtained at a single area of $A \sim 2.57 \AA^{2}$. Key: black and red lines - liquid quenches at fast and slow cooling rates, blue and green lines - from stress-induced amorphisation of the $4 / 8$ crystal and fast and slow rates. 


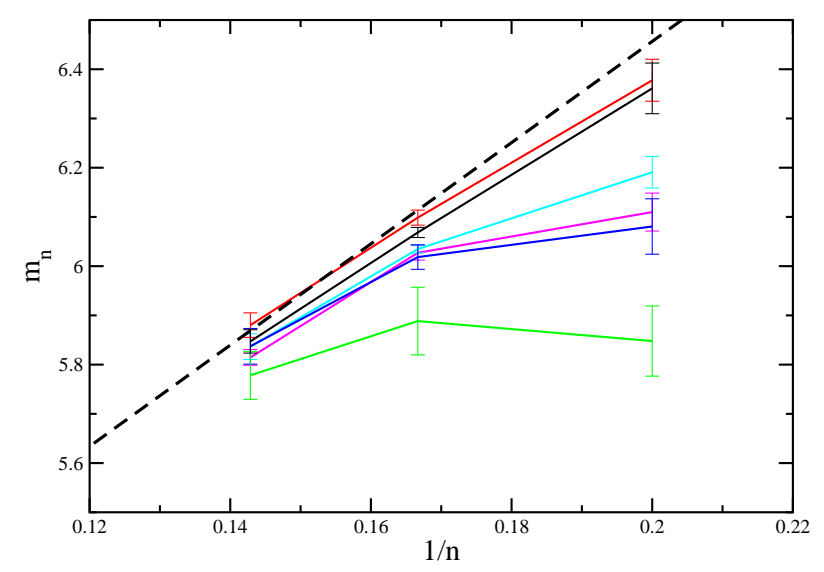

Figure 18. The mean size of the neighbouring rings with respect to a given central ring of size $n$ obtained at a single area of $A \sim 2.57 \AA^{2}$ under a range of cooling conditions. The Aboav-Weaire Law predicts a linear relationship (dashed line). Key (from most rapid to slowest cooling rates): green blue, magenta and cyan lines. For the black and red lines the four- and two-coordinate defects have been removed using the T1 and T2 mechanisms.
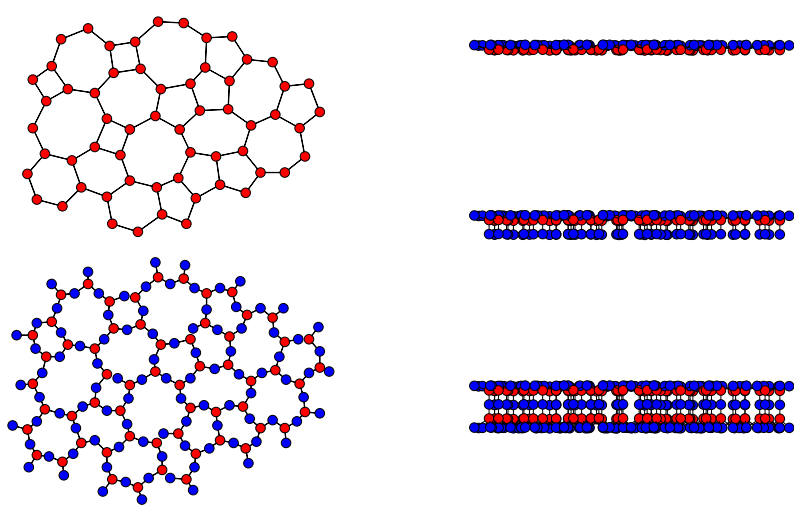

Figure 19. Molecular graphics schematic using Zachariasen's original coordinates [6] to construct a bilayer of $\mathrm{SiO}_{2}$. Key: blue - O atoms, red - $\mathrm{Si}$ atoms. The original configuration is shown in the bottom left. The right images show the effect of placing the $\mathrm{Si}$ atoms at the apices of a pyramid (top), adding a central layer of bridging $\mathrm{O}$ atoms (centre) and finally connecting a mirror image of pyramids (bottom). 

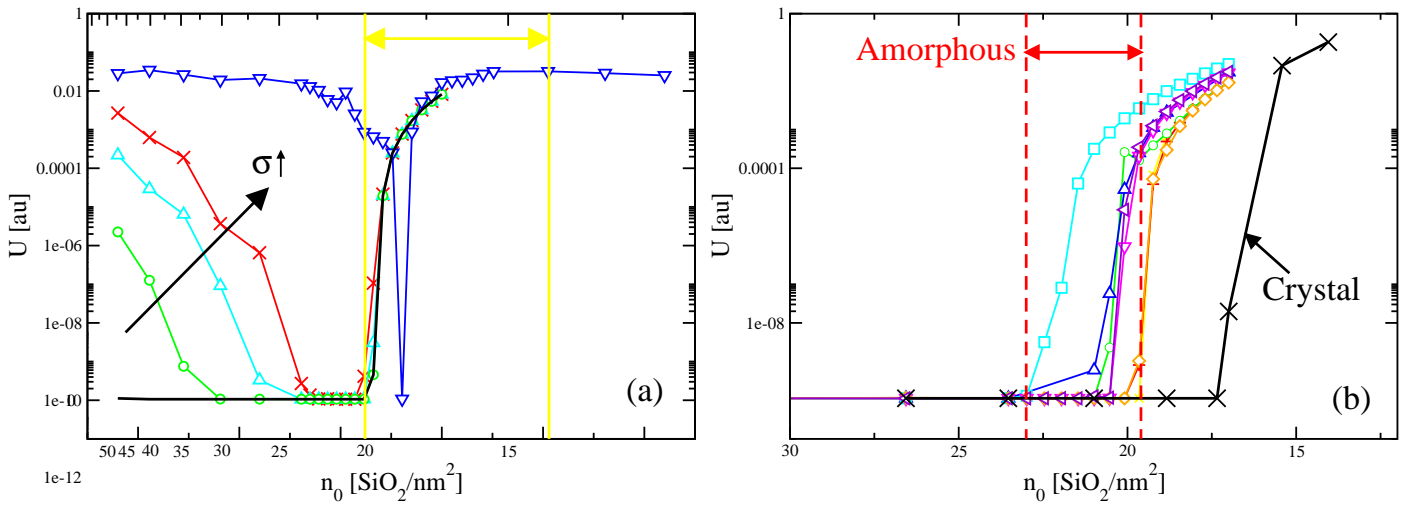

Figure 20. (a) The evolution of the potential energy for a fully relaxed bilayer of $\mathrm{SiO}_{2}$ as a function of area. Key: black line - harmonic potential only. The green, cyan and red lines show the effect of introducing an increasingly significant shifted 24-12 repulsive potential. The blue line shows the energy obtained from the full electrostatic TS potential [130]. The experimentally-determined density range is highlighted by the yellow lines and arrow. (b) The energy as a function of number density for five amorphous bilayer configurations and the ideal (six-membered rings) crystal structure, determined using a purely harmonic potential. The amorphous configuration energies can all be driven to zero above critical densities but, importantly, these densities vary with configuration as highlighted by the dashed red lines. 

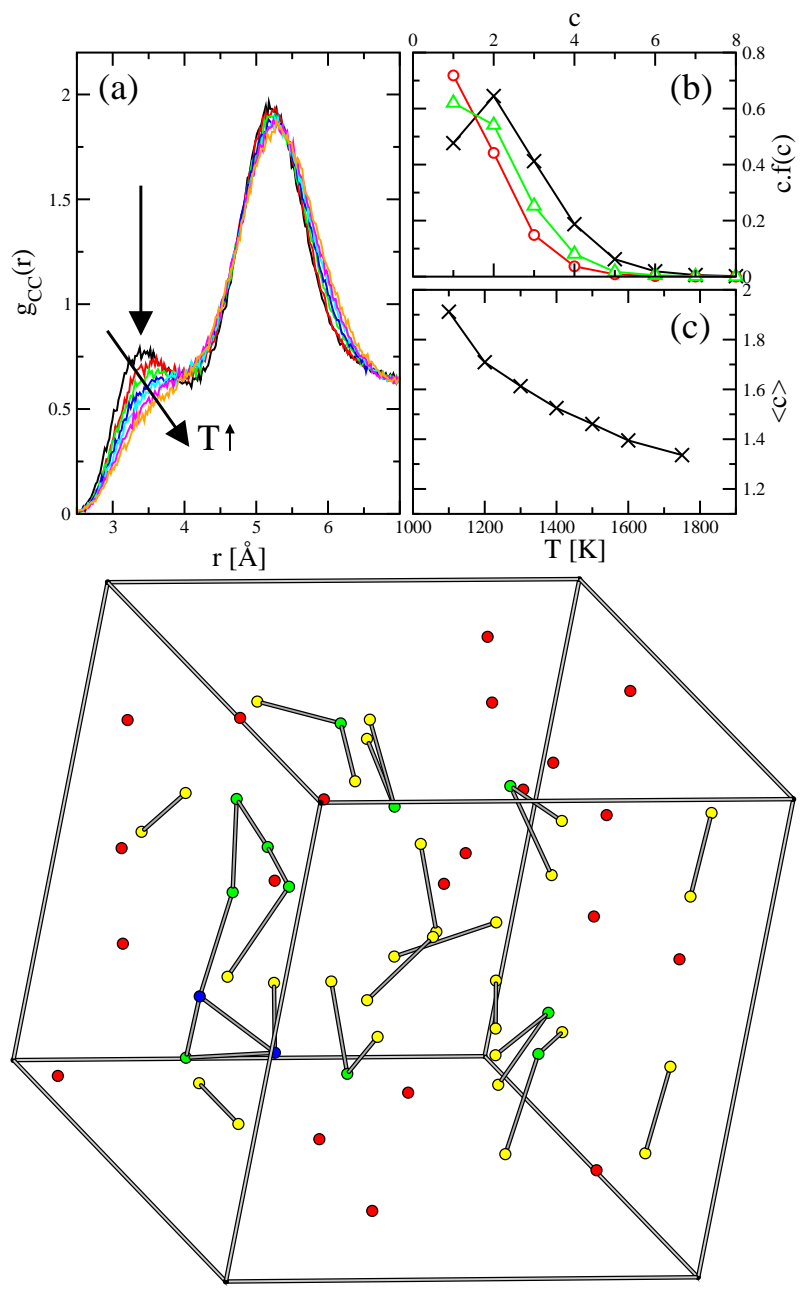

Figure 21. (a) The partial radial distribution function for the carbon-carbon separations, $g_{C C}(r)$, obtained from simulations of molten $\mathrm{Na}_{2} \mathrm{CO}_{3}$ over a temperature range from $T=1100 \mathrm{~K}$ to $T=1700 \mathrm{~K}$. As the temperature is increased the highlighted feature at $r \sim 3.3 \AA$ transforms from a well-defined peak to a shoulder. (b) The distribution of C-C chain lengths shown at three temperatures (black $-T=1100 \mathrm{~K}$ green $-T=1400 \mathrm{~K}$ red $-T=1700 \mathrm{~K}$ ). (c) Mean C-C chain lengths as a function of temperature. (d) Molecular graphics "snapshot" of a low temperature $(T=1100 \mathrm{~K})$ $\mathrm{Na}_{2} \mathrm{CO}_{3}$ configuration showing the $\mathrm{C}$ atoms only. $\mathrm{C}$ atoms separated by $r<4 \AA$ (corresponding to the first local minimum in $g_{C C}(r)$ as shown in panel (a)) are joined by a line and the $\mathrm{C}$ atoms are coloured according to their environment. Key: red - zero coordinate, yellow - singly-coordinate, green - two-coordinate, blue - three-coordinate. 